\title{
A Study of the Chemical, Mechanical, and Surface Properties of Thin Films of
} Hydrogenated Amorphous Carbon

\author{
Gilroy John Vandentop
}

\author{
(Ph.D. Thesis)
}

Department Of Chemisiry

University of California

\author{
and \\ Materials and Chemical Sciences Division \\ Lawrence Berkeley Laboratory \\ University of California \\ Berkeley, CA 94720
}

This work was supported by the Director, Office of Energy

Research, Office of Basic Energy Sciences, Materials

Science Division, of the U.S. Department of Energy under

Contract No. DE-AC03-76SF00098. 


\title{
A Study of the Chemical, Mechanical, and Surface Properties of Thin Films of Hydrogenated Amorphous Carbon
}

\author{
by \\ Gilroy John Vandentop
}

\section{ABSTRACT}

Amorphous hydrogenated carbon $(\mathrm{a}-\mathrm{C}: \mathrm{H})$ films were studied with the objective of elucidating the nucleation and growth mechanisms, and the origin of their unique physical properties. The films were deposited onto $\mathrm{Si}(100)$ substrates both on the powered (negatively self-biased) and on the grounded electrodes from methane in an rf plasma $(13.56 \mathrm{MHz})$ at $65 \mathrm{~m}$ Torr and 300 to $370 \mathrm{~K}$. The films produced at the powered electrode exhibited superior mechanical properties, such as high hardness. A mass spectrometer was used to identify neutral species and positive ions incident on the electrodes from the plasma, and also to measure ion energies.

The effect of varying ion energy flux on the properties of a-C:H films was investigated using a novel pulsed biasing technique. It was demonstrated that ions were not the dominant deposition species as the total ion flux measured was insufficient to account for the observed deposition rate. As ion energy flux was increased, the hardness increased, the compressive stress increased, the concentrations of methyl and methylene groups decreased, and the optical gap decreased, demonstrating how ion energy flux controls the final properties of the films.

The interface between thin films of a-C:H and silicon substrates was investigated using angle resolved $x$-ray photoelectron spectroscopy. An silicon carbide layer was detected at the interface of a hard a-C:H film formed at the povered electrode. At the grounded electrode, where ion kinetic energy is low, no interfacial carbide layer was observed. The results indicated that the thickness of the interfacial carbide 
layer was controlled by the average penetration depth of plasma ions into the silicon substrate, as controlled by their kinetic energy. The presence of the interfacial carbide was likely responsible for the good adhesion of $\mathrm{a}-\mathrm{C}: \mathrm{H}$ films formed on silicon at the powered electrode.

Scanning tunneling microscopy and high energy electron energy loss spectroscopy was used to investigate the initial stages of growth of a-C:H films. On graphite substrates, films formed at the powered electrode were observed to nucleate in clusters approximately $50 \AA$ in diameter, while at the grounded electrode no cluster formation was observed. A novel $2 \times 2$ structure was observed at the surface of graphite which had been exposed to the methane plasma. On silicon substrates, placed at the powered electrode, cluster formation was not observed, and the films were flat within $3 \AA$ rms. 
To my father 


\section{Acknowledgements}

The time spent remembering and thanking all those who contributed to the completion of my work at Berkeley is probably the most difficult part of completing this dissertation for me. U.C. Berkeley, the Lawrence Berkeley Laboratory, and the Somorjai group in particular, provide an unique wealth of resources for graduate students. It is not possible to list even the small fraction of these resources wh ch I have been able to take advantage of. Therefore, I must limit my acknowledgements to only a handful of those who deserve recognition.

I would like to express my deepest thanks to my thesis advisor, Gabor A. Somorjai. From the day I met him, his enthusiasm and vision have been inspirational. I will never forget Gabor's willingness and drive to personally teach me how to be successful in scientific endeavors. I will always be indebted to him for the knowledge I have gained through his efforts.

Miquel Salmeron's experience and expertise has had a significant influence on this work. His willingness to allow me to invade the STM lab is most appreciated. The patience and enthusiasm of both Miquel and Frank Ogletree to teach me all I wanted to learn about the STM, and about physics and electronics in general, made my last year at Berkeley as challenging and rewarding as my first.

Without the intense concentration and unbelievably hard work of Prof. Mitsuo Kawasaki, only a fraction of this work could have been accomplished. Much of this work came out through a rewarding partnership we had during his stay in Berkeley. I arr most grateful for his friendship and guidance. Others who contributed to the work presented here include Ian Brown, Roger Nix, Pedro Nascente, Troy Wilson, Kazuhisa Kobayashi, Kirk Veirs, Mike Rubin, and Mike Hilton.

During my stay in Berkeley many special friendships were begun. The aspect of graduate school which I enjoyed the least was how short the period of time was between developing a friendship and being separated from that friend by thousands of miles. The warmth of Jose Carrazza towards both my wife and myself will always be missed. My friend and fellow Canadian Ian Harrison was a reliable source of sound advice and always willing to 'chat it up'. One day I will tecach Bruno Marchon how to pronounce Disneyland and instill in him a proper respect for 'the Great One'. I am 
sure that, in his heart, Troy Wilson knows that he is an even more aggressive driver than I.

I would like to thank all the members of the Somorjai group who made my time in graduate school enjoyable and rewarding, although I can only list a few. The blowout room provided daily entertainment, as I enjoyed relentlessly defeating Jim Powers, Kevin Williams, Bob Hwang, and all other challengers at the Somorjai group foosball table. Although Colette Knight and Sabrina Fu seldom played, they had the most lovely curtains hung there. The days at Stinson beach with Dan Strongin, Dave Zeglinski, the Beebe family, Tom Rucker, Dave Godbey, and Ellice Luh were always a lot of fun. Credit for the most effective method of stress relief, though, must go to all my friends at the art school and college avenue pickup basketball venues.

I would like to thank the technical staff in MCSD for all their help and technical expertise. Special mention is in order for Bob Wright, Dan Colomb and Chip Flor who were even willing to sample my homebrew, and listen to my rantings on the virtues of Canadians and the glories of ice hockey.

The people I mention here last are those closest to my heart, and whose influence can not be measured. I would like to express my love and thanks to my parents, my family, and my farnily in-law for all their love and support. My closest, friend, Peter Wamsteeker, has always been a rock to hold on to and a standard by which I could keep things in perspective. I need not express my feelings towards him. He knows them.

Lastly, and most importantly, I wish to thank my wife. The sacrifices she makes and the efforts she expends in order to make my ambitions successful are immeasurable. I love her dearly and look forward the next chapter of our life together.

The work presented in this thesis was supported in part by the Director, Office of Energy Research, Office of Basic Energy Sciences, Materials Science Division of the US Department of Energy, Contract No. DE-AC03-76F00098. Additional support was provided by Imperial Chemical Industries.

Berkeley, June 1990 


\section{Contents}

1 Introduction $\quad \mathbf{2}$

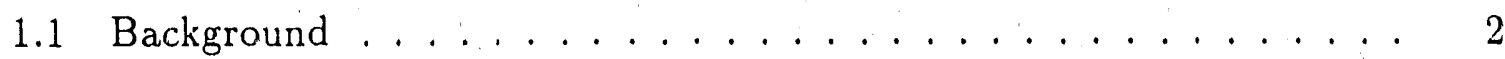

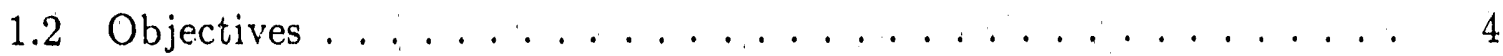

1.3 Organization of Thesis ................. 5

2 Experimental Procedures $\quad 7$

2.1 Plasma Assisted Chemical Vapor Deposition ............ . 7

2.1 .1 Background ................... 7

2.1.2 PACVD Apparatus .................. 8

2.1.3 : Plasrna Potential Profile ................ 9

2.1 .4 Plasma Analysis .................... 13

2.1 .5 Pulsed Biasing . . . . . . . . . . . . . . 20

2.2 Mechanical Properties . . . . . . . . . . . . . . 20

2.2.1 Knoop Hardness ................. 20

2.2 .2 Thickness and Stress . . . . . . . . . . . . . 23

2.2 .3 Scratch Adhesion Test ............... 23

2.3 Film Composition . . . . . . . . . . . . . . . . 24

2.3.1 Auger Electron Spectroscopy . . . . . . . . . . . . . . 24

2.3.2 X-ray Photoelectron Spectroscopy . . . . . . . . . . . . . 29

2.3.3 Fourier Transform Infrared Spectroscopy . . . . . . . . . . . 30

2.3.4 Ultraviolet/V sible Absorption Spectroscopy . . . . . . . . 32

2.3.5 Secondary Ion Mass Spectroscopy . . . . . . . . . . . 32

2.4 Film Structure . . . . . . . . . . . . . . . . 33

2.4.1 Scanuing Tunneling Microscopy ... . . . . . . . . . 33

2.4.2 High Resolution Electron Energy Loss Spectroscopy . . . . . . 36 
2.4 .3 Raman Spectroscopy . . . . . . . . . . . . . 37

3 a-C:H Film Properties

3.1 Introduction . . . . . . . . . . . . . . . . . 43

3.2 Growth Rate . . . . . . . . . . . . . . . . . 44

3.3 Mechanical Properties ................... 44

3.4 FTIR Spectra and Optical Gap ................ . 49

3.5 Discussion . . . . . . . . . . . . . . . . . 52

4 Plasma Characterization $\quad \mathbf{5 7}$

4.1 Introduction . . . . . . . . . . . . . . 57

4.2 Neutrals ........................... 57

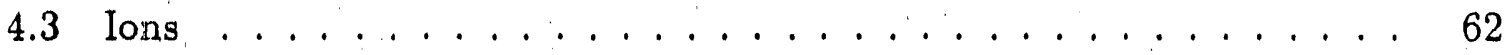

4.4 Discussion . . . . . . . . . . . . . . . . 68

5 Plasma Control $\quad 73$

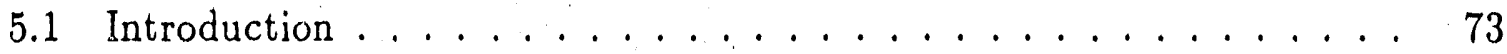

5.2 Pulsed Biasing Effect . . . . . . . . . . . . . . 74

5.2.1 Ion Current Density and Ion Energy Flux . . . . . . . . . 74

5.2 .2 Hardness and Deposition Rate . . . . . . . . . . . 75

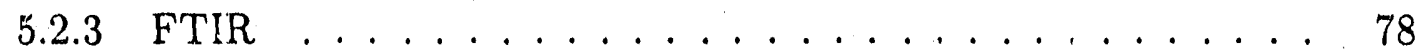

5.2.4 Optical and Raman Spectra .............. 78

5.3 Radical Scavenging . . . . . . . . . . . . . . . . 81

5.4 Discussion . . . . . . . . . . . . . . . . . 83

6 Interface Characterization $\quad 87$

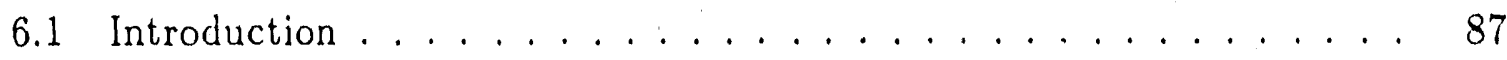

6.2 Angle.resolved XPS . . . . . . . . . . . . . . . . . 88

6.2.1 Interface Characterization . . . . . . . . . . . 88

6.2 .2 Effect of Ion Energy . . . . . . . . . . . . . . 97

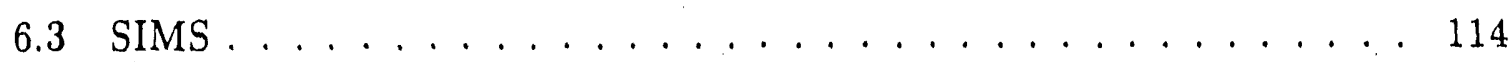

6.4 Discussion . . . . . . . . . . . . . . . . 114 
7 Nucleation and Growth by STM 117

7.1 Introduction . . . . . . . . . . . . . . 117

7.2 a-C:H on graphite . . . . . . . . . . . . . 117

7.3 a-C:H on $\mathrm{Si}(100) \ldots \ldots \ldots \ldots 131$

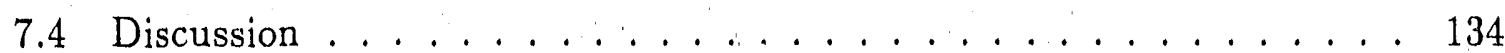

7.4 .1 a-C:H on graphite . . . . . . . . . . . . . . 134

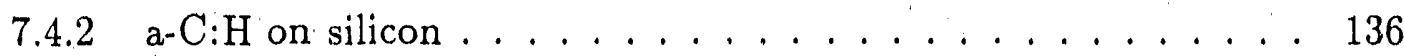

8 Summary 140 


\section{List of Figures}

2.1 Schematic diagram of the plasma deposition and mass analysis system. The substrate can be attached to either electrode. Pressure in the upper chamber is in the mTorr range, while in the lower chamber it is maintained in the $10^{-8}$ Torr range. . . . . . . . . . 10

2.2 Photograph of the plasma deposition and mass analysis system. ...

2.3 Self-bias voltage vs if power for the rf deposition system at a methane pressure of 65 mTorr. . . . . . . . . . . . . . . . . .

2.4 Plasma potential as it varies between the two electrodes. The powered electrode is at $0 \mathrm{~cm}$ and the grounded electrode is at $2 \mathrm{~cm}$. Potentials are based on Langmuir probe measurements and ion energy distributions. Boundaries between the glow region and the ion sheaths or dark spaces are based on visual inspection. . . . . . . . . . . . 14

2.5 Schematic diagram of the Faraday cup system used to measure the total ion flux. . . . . . . . . . . . . . . . .

2.6 Oscilloscope trace of the measured current during the pulsed bias condition. The equivalent shape of the background level and the total current level, measured with the ion orifice open, indicates that no measurable temporal variation in ion flux exists. . . . . . . . . .

2.7 Schematic of Knoop diamond indenter and the resultant indentation geometry. . . . . . . . . . . . . . . . . 22

2.8 Schematic of the Scratch Adhesion Test. . . . . . . . . . . . . 25

2.9 Photograph of UHV Scratch Adhesion Tester and tribometer. . . . . 26

2.10 Schematic diagram of the electronic transitions in the Auger process. 28

2.11 Conceptual depiction of the STM scanning system. . . . . . . . 35 
3.1 Deposition rate vs if power for the powered and grounded electrodes. Data points were taken for the powered and grounded electrodes in both upper and lower positions in the deposition apparatus to demonstrate the symmetry of the deposition rate with respect to gas flow. .

3.2 Summary of the mechanical properties of a-C:H films, diamond, and graphite, where $1 \mathrm{GPa}=102 \mathrm{~kg} / \mathrm{mm}^{2} \ldots \ldots \ldots \ldots$

3.3 Secondary electron microscope images of scratch tracks made with a Rockwell $\mathrm{C}$ diamond tip at a load of $830 \mathrm{~g}$, for films formed on the powered and the grounded electrodes. Little damage is apparent on the hard films formed on the powered electrode, while the soft film formed at the grounded electrode has suffered noticeable damage. . .

3.4 Peak to peak auger intensities for carbon and silicon as a function of argon ion sputter time for areas in the scratch tracks and away from the tracks of the areas shown in the previous figure. A large amount of intermixing is evident in the scratch track on the soft film formed on the grounded electrode. . . . . . . . . . . . . . .

3.5 FTIR spectra of a-C:H films formed on the grounded[(a) and (c)] and powered [(b) and (d)] electrodes. Film thicknesses for the grounded and powered electrode films were 1.27 and $2.15 \mu \mathrm{rn}$, respectively. The spectrum for the film formed on the grounded electrode shows absorptions due to $\mathrm{CH}_{3}$ which are not apparent in the powered electrode film spectrum. ..................... 50

3.6 Typical Raman spectrum for an a-C:H film. . . . . . . . . . 53

3.7 Typical SIMS spectra for an a-C:H film. . . . . . . . . . 54

4.1 Mass spectra of neutrals incident on the grounded electrode (GE) and the powered electrode $(\mathrm{PE}) \ldots \ldots \ldots \ldots$

4.2 Experimental and calculated intensities of the mass spectrometric intensities in the cracking of various $\mathrm{C}_{2} \mathrm{H}_{x}$ species. Intensities are calculated based on the optimum concentration ratios (calculated as $\left[\mathrm{C}_{2} \mathrm{H}_{6}\right]$ $\left.:\left[\mathrm{C}_{2} \mathrm{H}_{4}\right]:\left[\mathrm{C}_{2} \mathrm{H}_{2}\right]=0.45: 0.27: 0.28\right)$ and the cracking pattern spectra of the pure gases taken with the same apparatus. . . . . . . . . . 
4.3 Partial pressures of neutral species above the substrate at the GE and the PE. . . .............................. 61

4.4 Relative intensities of the mass $12-15$ signals due to neutral radical species, for the $\mathrm{GE}$ and the $\mathrm{PE}$. The $\mathrm{CH}_{3}$ radical is the predominant radical species in the plasma. . . . . . . . . . . . . .

4.5 Effect of direct path shielding on the detected neutral radical intensities. Intensities with and without a shield are shown. The lack of a measurable change in the 15 amu signal intensity indicates a small. sticking coefficient for the methyl radicals with the chamber walls. . .

4.6 Mass spectra of ions incident on the GE and the PE. The intensity for the grounded electrode spectrum has been magnified by a factor of 10 .

4.7 Ion signal intensity vs ion energy for the total ions, $\mathrm{H}_{2}^{+}$, and $\mathrm{CH}_{3}^{+}$ions incident on the grounded electrode (open shapes) and the powered electrode (solid shapes). The energies with which the ions strike the powered electrode are $350 \mathrm{eV}$ greater than those measured at the mass spectrometer and listed here. . . . . . . . . . . . . .

5.1 Apparent ion flux on the substrate for various deposition conditions.

5.2 The upper plot shows the a-C:H deposition rate as a function of ion energy flux. In the lower plot, the hardness and compressive stress of the a-C:H films is plotted as a function of ion energy flux. . . . . . .

5.3 Concentrations of methylene $\left(-\mathrm{CH}_{2}-\right)$ and methyl $\left(-\mathrm{CH}_{3}\right)$ groups in a$\mathrm{C}: \mathrm{H}$ films as a function of ion energy flux. . . . . . . . . . .

5.4 Changes in the optical gap of a-C:H films as a function of ion energy flux.

5.5 Effect of nitric oxide addition on the deposition rate at the PE and the GE. Radical scavenging by $\mathrm{NO}$ decreases the deposition rate dramati-

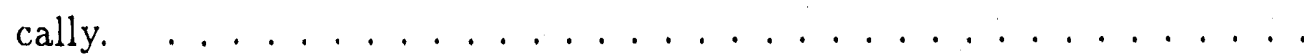

5.6 Mass spectra of ions incident on the powered electrode for $0 \% \mathrm{NO}$ feedgas concentration and for $20 \%$ NO. The presence of the radical scavenger has a relatively small effect on ion flux. . . . . . . . . 
6.1 Si $2 p$ spectra for various deposition times on $\mathrm{Si}(100)$ placed on the powered electrode, where a hard a-C:H film is produced. (a) electron takeoff angle $=60^{\circ}$. (b) electron takeoff angle $=15^{\circ} . \ldots \ldots$

6.2 C 1s spectra for various deposition times on $\mathrm{Si}(100)$ placed on the powered electrode. (a) electron takeoff angle $=60^{\circ}$. (b) electron takeoff ar gle $=15^{\circ} \ldots \ldots \ldots \ldots \ldots$

6.3 Relationship between the peak areas of Si $2 \mathrm{p}$ and $\mathrm{C} 1$ s signals associated with the interfacial silicon carbide layer. The data are collected for various deposition times ( 5 to $20 \mathrm{~s}$ ) and electron takeoff angles $\left(60^{\circ}\right.$ to $15^{\circ}$ ). The intensity ratio of near unity suggests the silicon carbide layer is approximately stoichiometric. . . . . . . . . . . . . .

6.4 XPS spectra showing the $\mathrm{O} 1 \mathrm{~s}$, Si $2 \mathrm{~s}$, and $\mathrm{Si} 2 \mathrm{p}$ peaks at electron takeoff angles of $60^{\circ}$ and $15^{\circ}$. Deposition lime is $20 \mathrm{~s}$ on the powered electrode. The intensity is normalized to the $\mathrm{C} 1 \mathrm{~s}$ peak to account for the arigle dependant instrurnent factor. The lack of attenuation of the oxygen signal indicates that oxygen is not associated with the interface. 94

6.5 Dependence of $R(\theta)$ as defined in the text from the ratio of the chemically shifted Si $2 p$ peak (from $\mathrm{SiC}$ ) to the pure $\mathrm{Si} 2 \mathrm{p}$ peak (from the Si substrate), on $1 / \sin \theta \cdot \theta$ represents the electron takeoff angle. . . .

6.6 A Si 2 p spectrum from a soft a-C: $\mathrm{H}$ film on silicon at electron takeoff angle of $15^{\circ}$. Deposition time is $20 \mathrm{~s}$. A suboxide of silicon is present at the interface of these films at submonolayer levels. . . . . . . . .

6.7 Si $2 \mathrm{p}$ spectra of a-C:H films as a function of self bias voltage. These spectra were taken at an electron take off angle of $30^{\circ}$, with $\mathrm{rf}$ power rahging from 10 to $100 \mathrm{~W}$. . . . . . . . . . . . . 100

6.8 C 1 s spectra of a-C:H ilms as a function of self bias voltage. These spectra were taken at a take off angle of $60^{\circ}$ for $\mathrm{rf}$ powers ranging from 10 to $100 \mathrm{~W}$

6.9 Relationship between the peak area of Si $2 \mathrm{p}$ and $\mathrm{C} 1 \mathrm{~s}$ signals associated with the interfacial carbide. 
6.10 Dependence of $R(\theta)$ as defined in the text on $1 / \sin \theta$, plotted for a series of self bias voltage conditions. The interface thickness, as calculated from the slope of the best fit lines are also given in the figure. . . . . 104

6.11 Calculated thickness of the silicon carbide interface as a function of self bias voltage. The solid line gives the results of the theoretical penetration depth of $\mathrm{C}^{+}$ions into silicon as a function of ion energy, as calculated using TRIM code. . . . . . . . . . . . . 105

$6.12 \mathrm{Si} 2 \mathrm{p}$ spectra for a-C:H films grown on silicon under various pulsed biasing conditions. For comparison, the spectra for films formed on the grounded and powered electrodes are included. . . . . . . . . . 107

6.13 Relationship between the peak area of the Si $2 p$ and $C$ 1s signals associated with the is rfacial silicon carbide layer. The scatter of the points is relatively large, but, all the data falls in the regime of silicon rich composition. . . . . . . . . . . . . . . . . . . . . . . 109

6.14 The variation in the linearity of the data as plotted as a function of the parameter $\phi$, which denotes the ratio of pure silicon to silicon carbide in the interface region, using the model described in the text. . . . . 112

6.15 Calculated thickness of the silicon carbide interface as a function of pulsed biasing voltage.

7.1. 1000 by $1000(\AA)^{2}$ STM topographic image of a-C:H formation on graphite. This image is of an area of relatively low cluster density in

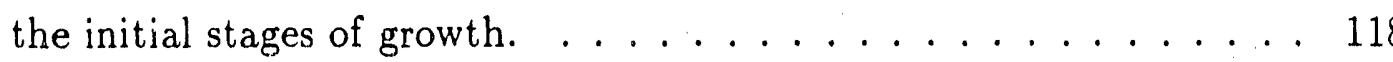

7.21000 by $1000(\AA)^{2}$ STM topographic image of a-C:H grown on graphite. High cluster density area. . . . . . . . . . . . . . . . 120

7.3200 by $200(\AA)^{2}$ STM topographic image of clusters of a-C:H on a graphite surface. The regular periodicity of the graphite substrate is visible in between the amorphous looking clusters. . . . . . . . . . . 121

7.425 by $25(\AA)^{2}$ STM topographic image of a cluster of a-C:H as formed on a graphite substrate. 
7.5 High resolution electron energy loss spectra of a graphite blank, and of a film of a-C:H grown on graphite. The broad peaks at $1200-1400$ $\mathrm{cm}^{-1}$ and around $2935 \mathrm{~cm}^{-1}$ correspond to $\mathrm{C}-\mathrm{H}$ bending and stretching vibrations, respectively. . . . . . . . . . . . . . . . . 124

7.6 Three dimensional view of a topographic STM image taken of the initial stages of a-C:H growth at the powered electrode. Cluster formation during the initial stages of growth is observable. . . . . . . . . 125

7.7 Three dimensional view of topographic STM image taken of the initial stages of a-C:H growth at the grounded electrode. No cluster formation is observable.

7.8200 by $200(\AA)^{2}$ topographic STM image displaying a number of areas where a $2 \times 2$ periodicity is observed on the surface of graphite which has been exposed to a methane $\mathrm{rf}$ plasma. . . . . . . . . 128

7.981 by $81(\AA)^{2}$ inset of the previous figure. The double graphite periodicity is clear in the center area of the image. In the lower left corner an area of regular graphite periodicity is observable for comparison.

7.1050 by $50(\AA)^{2}$ area of graphite exposed to a methane ri plasma for 6 seconds at the grounded electrode. A $2 \times 2$ periodicity in the structure is observable in the center of the image towards the left. . . . . . 130

7.111800 by $1800(\AA)^{2}$ topographic STM image of a typical surface of a silicon blank substrate.

7.121800 by $1800(\AA)^{2}$ topographic STM image of a typical surface of a$\mathrm{C}: \mathrm{Fi}$ on silicon. The silicon substrate, in this case, was exposed to the methane plasma for 10 seconds at the powered electrode. 


\section{Chapter 1}

\section{Introduction}

\subsection{Background}

Amorphous hydrogenated carbon (a-C:H) films possess unique and interesting properties which, in industry, have been exploited in many ways. The hardness and wear resistance of $\mathrm{a}-\mathrm{C}: \mathrm{H}$ have led to the use of films of this material as protective coatings, most notably as wear resistant coatings to protect computer hard discs. The material has also been used as an optical coating, both for antireflective and protective functions due to its relatively low absorption in the visible and infrared, and because of its rugged mechanical properties. a-C:H films have been synthesized using a variety of techniques including plasma assisted chemical vapor deposition (PACVD), ion beam deposition[1], and sputter deposition[2]. Although developments in the applications of a-C:H films are quite extensive, the state of the art, as far as understanding the origin of the valuable properties of this material and its atomic scale structure, is to date still rather primitive.

Amorphous hydrogenated carbon is often referrea to as diamondlike carbon. This name can be misleading due to recent developments in low pressure diamond film synthesis $[3,4]$. Although a- $\mathrm{C}: \mathrm{H}$ shares some of the attractive properties of diamond, the structures of these two materials are radically different. Diamond films contain very little hydrogen. Diamond films are commonly grown at high temperatures in an atmosphere of excess hydrogen. The films are typically made up of microcrystals of diamond with diameters of tens of microns. a-C:H films, in contrast, contain as much as 50 at.\% hydrogen, and are generally grown close to room temperature from hydrocarbon feed gases, without the addition of excess hydrogen. The films contain 
both $\mathrm{sp}^{2}$ and $\mathrm{sp}^{3}$ bonded carbon, and are thought to be made up of crosslinked clusters of $\mathrm{sp}^{2}$ carbon[5]. The $\mathrm{sp}^{3}$ bonded carbon is thought to be associated mainly with the crosslinking structures, and is not in the form of diamond microcrystals. a-C:H films and diamond films are, therefore, two structurally distinct materials and should not be confused with each other. Henceforth, in order to avoid confusion, the terrn 'diamondlike carbon' will not be used in this text.

A number of comprehensive reviews exist $[6,7,8]$, which demonstrate the tremendous amount of effort which has been devoted to the study of a-C:H films. Almost every conceivable physical property of a-C:H has been measured, and some plausible models for the structure of the material exist $[9,10]$. Inferring the local structure of an amorphous material from measurements of the material's physical properties is a difficult task, and discussions regarding the real structure will undoubtedly continue.

One of the reasons that such great interest in the structure of a-C:H films exists, is that knowledge of the structure should help us understand the origin of the attractive physical properties of a- $\mathrm{C}: \mathrm{H}$ films. As a- $\mathrm{C}: \mathrm{H}$ films have been deposited to date, their properties are ideal for some applications. But there are, of course, limitations to the applicability of a-C:H films. For example, the adhesion of a-C:H films onto many metal substrates is poor, thus applications of a-C: $\mathrm{H}$ films as wear resistant coatings on tools have not been possible. Also, the mechani.al and optical properties of the films have not been controlled independently, thus achieving the optimum in performance in both areas simultaneously has not been possible.

An understanding of the structure of a-C:H films would obviously contribute a great deal towards attaining greater control over the physical properties of the films. However, what is most lacking presently is an understanding of the mechanisms of nucleation and growth of a-C:H films. This is clear from the notable absence, in review articles, of discussions concerning growth mechanisms. The reason behind this lack of discussion, as expected, is a lack of experimental results; results which shed light on the identity of precursor species, or on the mechanisms of nucleation and growth. This area of relatively poor understanding, will likely be the limiting step for future advancement in practical uses for a-C:H films.

Many studies have been conducted in order to determine what the necessary 
plasma conditions are which yield carbon films with the desired properties. What has been lacking is an understanding of the chemistry occurring in the plasma deposition systern, and how the reaction processes change with variations in plasma conditions. A better understanding of the mechanisms of growth is crucial, so that limitations in the film properties can be overcome.

\subsection{Objectives}

The objective of this research is to develop an understanding of the growth process of a-C:H films produced from methane by PACVD. Identification of the precursor species and their respective roles in the growth mechanism of a- $\mathrm{C}: \mathrm{H}$ films is key to developing such an understanding. A comprehensive study of the growth process must also include investigations probing the initial stages of growth and the properties of the interface.

In order to achieve these objectives, studies of the process of PACVD, as well as the resultant film properties were conducted. The active species in the plasma, including the radical and ionic species, were detected by means of mass spectrometry. Methods of control over the relative flux of radical and ionic species were developed, so that their respective effects on the final physical properties of the films could be investigated separately. The ion energy flux was controlled using a pulsed biasing method, by which means the energy of the incident ions could be controlled separately from the radical flux. Radical scavenging studies were employed in order to vary the radical flux separately from the ion flux. Utilizing these techniques, the respective roles of ions and radicals in the formation mechanism and their effects on the final properties of the films could be determined. Film properties investigated include hardness, scratch resistance, density, compressive stress, as well as infrared and optical absorbance spectra. Angle resolved x-ray photoelectron spectroscopy was used to investigate the composition and thickness of the interface. The effect of varying incident ion energy on the thickness of the interface was also investigated. Nucleation phenomena and the initial stages of growth were investigated using scanning tunneling microscopy and high resolution electron energy loss spectroscopy.

By using this large variety of scientific techniques, and performing new experi- 
ments which shed light on plasma processes, this research is intended to concentrate on the chemistry which takes place in the plasma and at the film surface. The objective is to develop a greater understanding of the mechanisms involved in a-C:H film formation, and thus open up possibilities for a superior control over film properties.

\subsection{Organization of Thesis}

This dissertation is organized into eight chapters. The first chapter discusses the objectives of the research and how they compliment what has been done in the past. The second chapter gives a brief description of the many techniques utilized throughout the course of the research and what can be expected to be learned from each of them. The remaining chapters deal with results and discussion. Some of the physical properties of the films as deposited by PACVD are given in chapter 3 . Differences in these properties between films formed on the powered versus the grounded electrodes led to questions regarding the active species present at the growing surface. Mass spectrometric investigations of the plasma, carried out in order to answer these questions, are described in chapter 4 . In order to understand the mechanisms occurring in the plasma deposition process, it was necessary to develop means of independently controlling the fluxes of active plasma species. In chapter 5 these methods of control are described, namely pulsed biasing and radical scavenging techniques. The results obtained, using these techniques, support a growth mechanism involving both radicals and ions. The interface of $\mathrm{a}-\mathrm{C}: \mathrm{H}$ films on silicon substrates was investigated using angle resolved $x$-ray photoelectron spectroscopy, and the results of this work are given in chapter 6 . Chapter 7 describes investigations of the initial stages of growth of

these films using scanning tunneling microscopy and high resolution electron energy loss spectroscopy. In the eighth and final chapter of this thesis, the results are summarized and the insight gained by this work into the process of a-C:H film formation is further discussed. 


\section{Bibliography}

[1] S. Aisenberg and R. Chabot, J Appl. Phys. 42, 2in53 (1971).

[2] J. Zelez, J. Vac. Sci. Tech. A 1, 305 (1983).

[3] J.C. Angus and Cliff C. Hayman, Science 241, 913 (1988) and references therein.

[4] C.V. Deshpandey and R.F. Bunshah, J. Vac.' Sci. Tech. A 7, 2294 (1989) and references therein.

[5] M.A. Tamor and C.H. Wu, J. Appl. Phys. 67, 1007 (1990) and references therein.

[6] H. Tsai and D.B. Bogy, J. Vac. Sci. Tech. A 5, 3287 (1987), and references therein.

[7] J.C. Angus, P.Koidl, and S. Domitz, in Plasma Deposited Thin Films, edited by J. Mort and F. Jansen (CRC, Boca Raton, FL, 1986).

[8] J. Robertson, Adv. Phys. 35, 317 (1986).

[9] M.A. Tamor, C.H. Wu, R.O. Carter, III, and N.E. Lindsay, Appl. Phys, Lett. 55, 1388 (1989).

[10] J. Robertson and E.P. O'Reilly, Phys. Rev. B 35, 2946 (1987). 


\section{Chapter 2}

\section{Experirnental Procedures}

\subsection{Plasma Assisted Chemical Vapor Deposition}

\subsubsection{Background}

Plasma assisted chemical vapor deposition has been widely used for the deposition of thin films. Reactive species are generated in the plasma, which can act as precursor species for film deposition. In traditional chemical vapor deposition, only ground state guseous species are available at the substrate surface to act as film precursors and very high substrate temperatures are often necessary to support reaction. The motivation for using a plasma is that the reactive species formed in a plasma often allow the necessary surface reactions and resultant film deposition to occur at much lower substrate temperatures.

A plasma is a partially ionized gas in which collisions occur with sufficient frequency so that long range electric coupling exists between ions. Typical gas pressures capable of sustaining a plasma are between 50 mTorr and 5 Torr. Ions, free radicals, and electronically excited species are created in the plasma by electron impact ionization of the ground state plasma gas, and can act as reactive precursor species in film growth.

The energy required for the maintenance of this collection of reactive species is provided by an electric field. This field can be applied in either a d.c. or a.c. mode. A.C. fields are required if the substrates to be used are electrically insulating. Energy is transferred from the electric field to the plasma principally by the acceleration of plasma electrons. As mentioned, these electrons collide with the ground state 
gaseous species to produce reactive ions, radicals, and electronically excited species. The energy transferred into the plasma from the electric field must be balanced by an equal energy transfer out of the plasma. This outward energy transfer is mediated by the energetic species generated in the plasma. These species collide with the : alls of the plasma chamber and with the substrate, thus imparting their excess energy to film growth processes, and possibly the formation of metastable phases. Energy is also usually emitted in the form of radiation during ion-electron recombination or elec' ronic relaxation processes, hence the characteristic glow of a plasma.

The transport of neutral species to the surface can be explained relatively simply, in terms of the physics of diffusion and adsorption. The behaviour of the plasma ions and electrons is inherently more complicated due to the effects of the varying electric fields. Since plasma electrons have a much greater mobility than the more massive ions, the initial flow of electrons from the plasma to the electrodes is much greater than the flow of positive ions. This depletes the electron density in the plasma. As a result, the potential of the bulk of the plasma is increased and becomes positive with respect to the electrode potential. A potential gradient between the plasma and the electrode is formed. This is known as the ion sheath or dark space[1,2]. It is darker than the center of the plasma, or the glow region, because the depletion of electrons in the sheath results in $a$ lower probability for luminescent ion electron recombination processes.

The potential gradient in the ion sheath retards the flux of electrons to the electrodes. Positive ions which diffuse into the sheath are accelerated into the electrode or substrate. The resultant ion b'mbardment can result in chemical and structural modification of the surface. Secondary electrons, created at the electrode during the ion-substrate collisions, are accelerated into the plasma by the electric field in the sheath. The accelerated electrons complete the cycle via collisions with the ground state gaseous species in the plasma, creating new reactive ions and radicals.

\subsubsection{PACVD Apparatus}

A parallel plate electrode system is often used when depositing onto planar substrates. In this configuration, electric fields are highly uniform as compared with other 
geometries, and the ratio of plasma volume to electrode surface area is minimized to make most efficient use of energy input. An example of an alternative geometry is the inductively coupled coil configuration, which is useful for deposition onto nonplanar substrates and for large batch jobs [3].

The films in this study were synthesized by PACVD in an rf apparatus (frequency 13.56 MHz) with a parallel plate cunfiguration as illustrated in Fig. 2.1 and Fig. 2.2. The plates were $8.75 \mathrm{~cm}$ in diameter with a separation distance of $2.0 \mathrm{~cm}$. The plates were housed in a stainless steel chamber pumped by a Seargent Welch model 1397 mechanical pump to a base pressure less than 10 mTorr, as measured with a Baration type 227AHS-C-10 capacitance manometer. Methane gas (Matheson 99.5\%) was used as the feedstock. Gases were introduced into the plasma region through an orifice in the upper electrode. Gas flow rates were measured with Porter mass flow controllers.

Pressures given in this paper are those measured before ignition of the plasma, unless otherwise stated. Typically, rf power was applied to the lower electrode while the upper electrode was grounded, but the connection was reversed when mass spectromel ric analysis of the species impinging on the grounded electrode was desired. Single crystal $\mathrm{Si}(100)$ wafers, $0.338 \mathrm{~mm}$ thick, were used as substrates. To removo the native oxide, the wafers were etched in $49 \% \mathrm{HF}$ immediately before being attached to the electrodes. Glass slides were used as substrates for samples to be used for optical transmission measurements. The glass slides were cleaned with methanol before deposition.

\subsubsection{Plasma Potential Profile}

A quantity of key interest in this study is the potential drop between the plasma glow region and the sample surface. This potential drop determines the energy with which positive ions will strike the sample surface. For a sample on the rf powered electrode, the potential drop is the difference between the plasma potential and the self-bias potential at that electrode. The of powered electrode acquires a negative self-bias voltage with respect to the plasma potential, due to the large difference in ion versus - ectron mobilities[1]. For our parallel plate electrode system, the self-bias voltage varies with applied $r f$ power as shown in Fig. 2.3. 


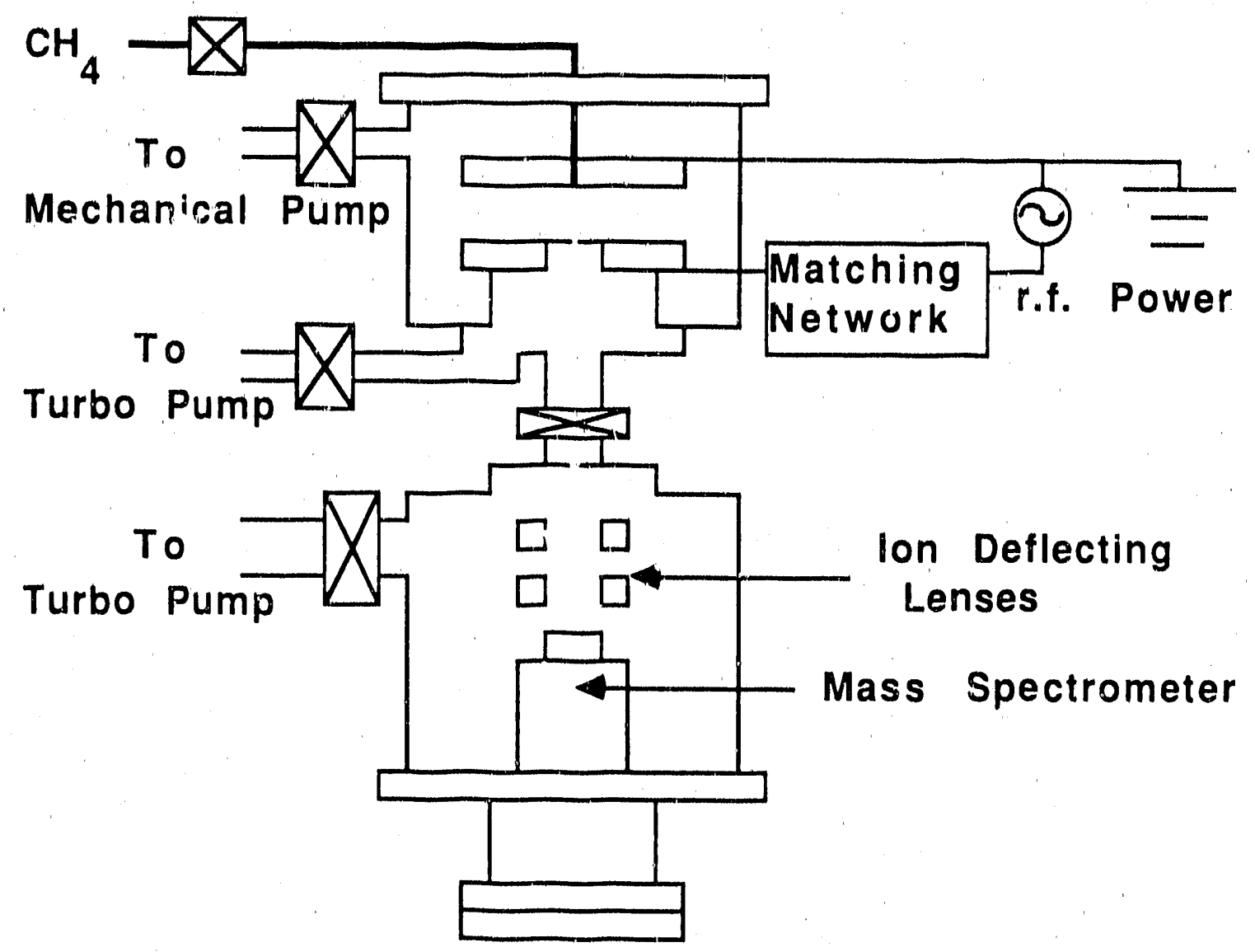

XBL $896-2520$

Figure 2.1: Schematic diagram of the plasma deposition and mass analysis system. The substrate can be attached to either electrode. Pressure in the upper chamber is in the mTorr range, while in the lower chamber it is maintained in the $10^{-8}$ Torr range. 


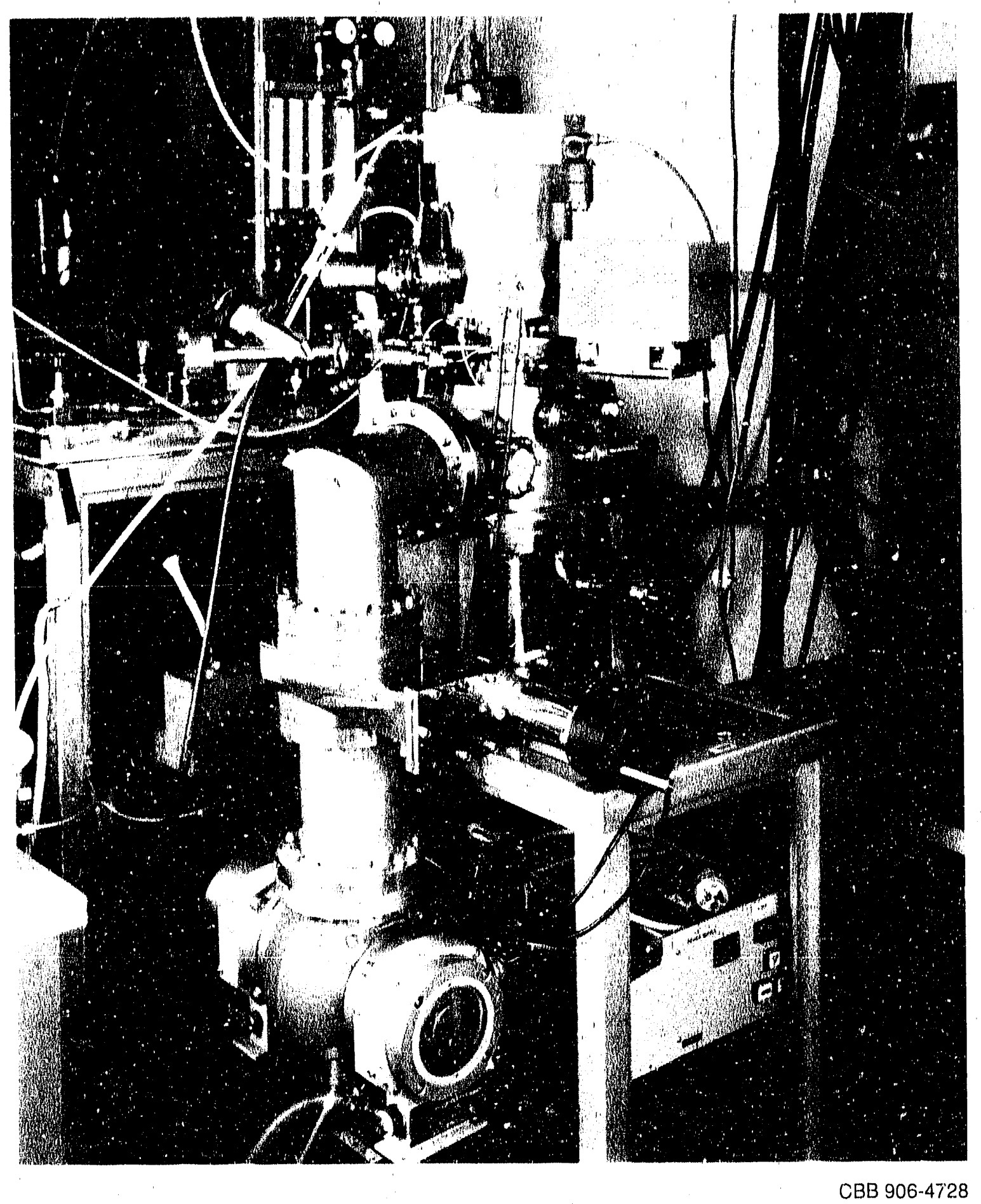

Figure 2.2: Photograph of the plasma deposition and mass analysis system. 


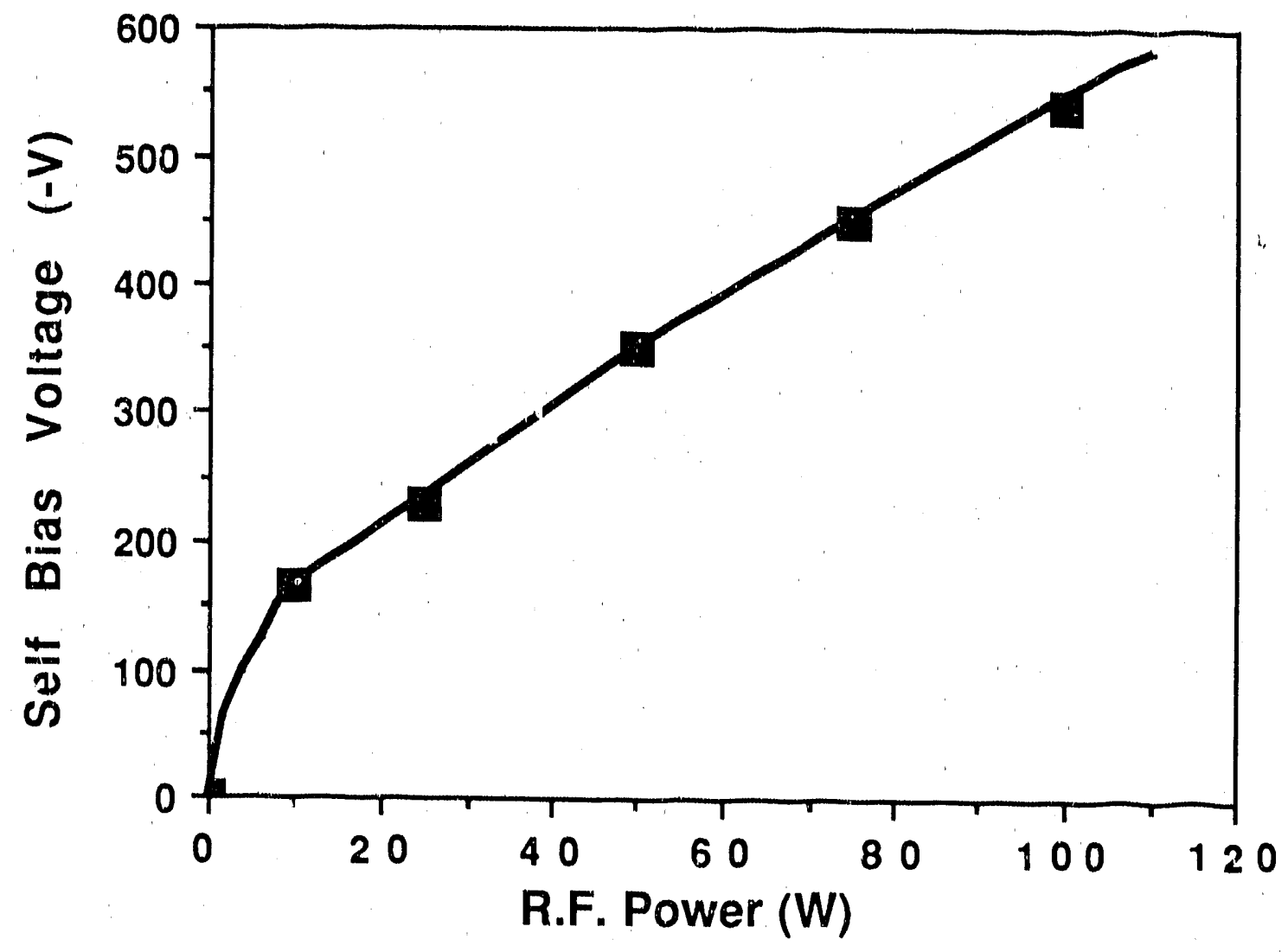

XBL 896-2521

Figure 2.3: Self-bias voltage vs if power for the rf deposition system at a methane pressure of 65 mTorr. 
When the plasma is ignited, a steady state potential distribution is established between the electrodes. Fig. 2.4 shows how the plasma potential is expected to vary across the inter-electrode gap for an $\mathrm{rf}$ power of $50 \mathrm{~W}$. The sheath width in this figure is based on a Debye shielding length (about $2 \mathrm{~mm}$ ), as estimated from an exponential curve fitting of the floating potential profile as measured with a Langmuir probe[4]. This is only a first approximation to the real sheath width, but Fig. 2.4 correlates well with the width of the cathode dark space $(\sim 1 \mathrm{~cm})$ which could be observed visually through a window in the plasma chamber. The plasma potential in the glow space in Fig. 2.4 is based on the average energy of ions incident on the grounded electrode, presented later in Fig. 4.7.

It is evident from Fig. 2.4 that positive ions created in the plasma region are accelerated towards both electrodes. However, the situation is very asymmetric, as positive ions striking the powered electrode can have as much as $400 \mathrm{eV}$ of energy, while those striking the grounded electrode will have an energy close to $15 \mathrm{eV}$.

\subsubsection{Plasma Analysis}

\section{Mass Spectroscopy}

In order to gain an understanding of the growth mechanism of a-C:H films, precursor species must be identified. Identification of reactive species present in a plasma is difficult. Theoretical modelling has beer used to predict the concentrations of plasma species[5]. However, reliable data for the reaction rates is not available for many of the possible reactions present in the plasma, so the accuracy of the theoretical predictions is not known. Analysis of the optical emission from the plasma can also be used to identify the species present[6], but only a select few of all the possible precursor species are detectable. Mass spectroscopy, on the other hand, is a sensitive and informative method for identifying species in the gas phase[7]. It can measure ions separately from the neutrals, and can detect the ionized parent and fragment ions of virtually any neutral species. Since most ionization cross sections are known or measurable, mass spectroscopy can give quantitative data on the concentrations of neutrals as well as ions.

Mass spectroscopy is a reliable and well known technique. However its application 


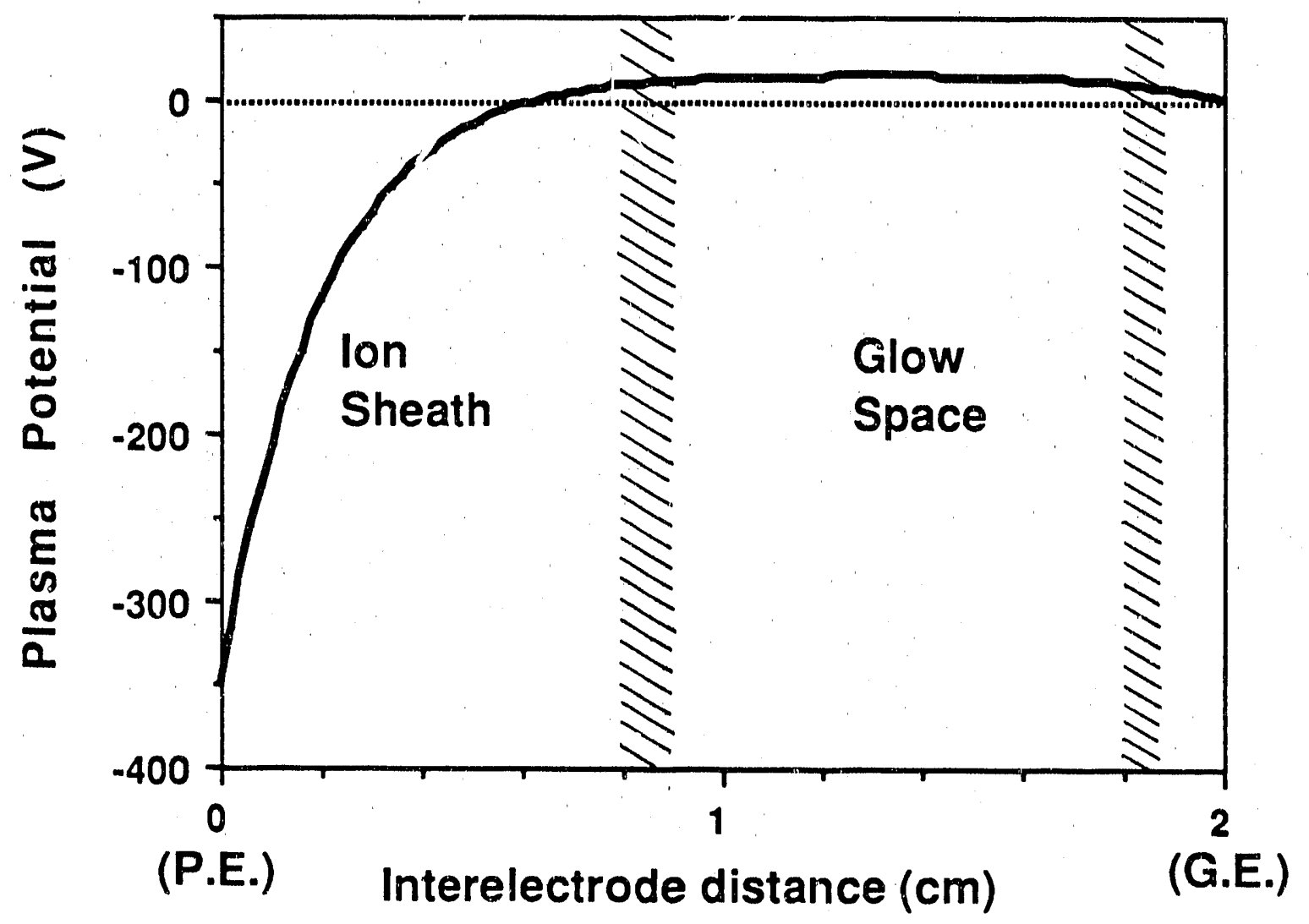

XBL $896-2522$

Figure 2.4: Plasma potential as it varies beiween the two electrodes. The powered electrode is at $0 \mathrm{~cm}$ and the grounded electrode is at $2 \mathrm{~cm}$. Potentials are based on Langmuir probe measurements and ion energy distributions. Boundaries between the glow region and the ion sheaths or dark spaces are based on visual inspection. 
for measuring plasma species is quite novel. The species of interest in this work include all chemical species which are incident on the growing sample surface during deposition. A small orifice at the sample location allows all the species normally impinging on the sample to be extracted from the plasma. The loss of plasma species through the orifice is assumed to ive a negligible effect on the plasma concentrations because of the small size of the orifice. The plasma species extracted through the orifice must travel to the mass spectrometer without collisions so that no reactions can occur between the time of extraction and the time of ionization or detection. Differential pumping was employed in order to keep pressures sufficiently low in the extraction system so that this criterion was met.

While the plasma ions are detected directly by the mass spectrometer, the plasma neutrals must first be ionized. This is achieved by electron impact ionization. Unfortunately, neutral molecules can not be exclusively and quantitatively ionized to a +1 charge. Molecular species undergo cracking reactions as a result of the electron impact process, and the ionic species actually detected include the various products of the numerous reaction pathways. This cracking phenomenon makes interpretation of the mass spectra obtained more difficult. However, since most cracking reaction probabilities are known or measurable, a reliable subtraction of signal resulting from secondary cracking species is possible. Thus the concentrations of the neutral species initially extracted from the plasma can be determined.

The mass spectrometric plasma analysis systern used in this work was designed and built in this laboratory and is schematically illustrated in Fig. 2.1 and Fig. 2.2. The plasma chamber was connected through a differentially pumped middle stage to a high vacuum chamber in which was installed a UTI model $100 \mathrm{C}$ mass analyzer along with ion deflection lenses used to measure the ion energy distributions. The first orifice through the lower electrode in the plasma chamber was approximately $100 \mu \mathrm{m}$ in diameter, while the second orifice, between the middle stage and the lower high vacuum chamber, was about $1 \mathrm{~mm}$ in diameter. Pressure was maintained on the order of $10^{-8}$ Torr in the lower chamber. The ion deflection lenses were made of two metal plates, separated by $5 \mathrm{~mm}$, with the center holes $3 \mathrm{~mm}$ in diameter. To minimize undesirable off-axis deflection of ions, the upper plate was grounded and 
the retardation voltage was applied to the lower plate so that the ion retarding field was confined between the plates. For the same reason, these lenses were placed as close as possible to the mass analyzer.

The mass spectra for the neutral species were taken under the normal ionizer-on operation, with electron energy of $75 \mathrm{eV}$ and ion energy of $19 \mathrm{eV}$. While taking ion spectra, every component in the ionization chamber was grounded, except for an ion focusing lens (ionizer-off operation).

\section{Ion Flux Measurement}

The experimental setup used to measure the plasma ion flux incident on the lower electrode is shown in Fig. 2.5. A small hollowed cylindrical electrode was screwed into a glass tube, which held it about $5 \mathrm{~mm}$ below an orifice $(0.35 \mathrm{~mm}$ in diameter) located in the lower electrode. The pressure below the electrode orifice was maintained on the order of $10^{-6}$ torr. In order to attract the positive ions incident on the orifice area, and to repel plasma electrons, a bias voltage of -200 to $-800 \mathrm{~V}$ was applied to the ion collector. The area of acceptance of the ion collector was large enough to collect the ions effectively, however, the geometry is not optimal for minimizing the loss of secondary electrons, which could contribute to the measured current. Thus, the current measured using this system is, in effect, an upper limit on the real ion current.

The steady state current was measured using a digital D.C. ammeter. An A.C. measurement was also performed, where the potential drop across a standard resistance was monitored with an oscilloscope. By covering the orifice with a shield to remove the direct pathway of ions from the plasma to the collector, the background current was first measured, and then subtracted from the current measured without the shield in place. The net current divided by the area of the orifice thus reprisents an apparent ion flux incident on the lower electrode.

In order to calculate the time averaged ion energy flux from the ion current, we must also take into account possible temporal variations in the ion current. This is especially important for the ion flux measurements during pulsed biasing conditions. The ion current during the high voltage pulse is not necessarily equal to the current 


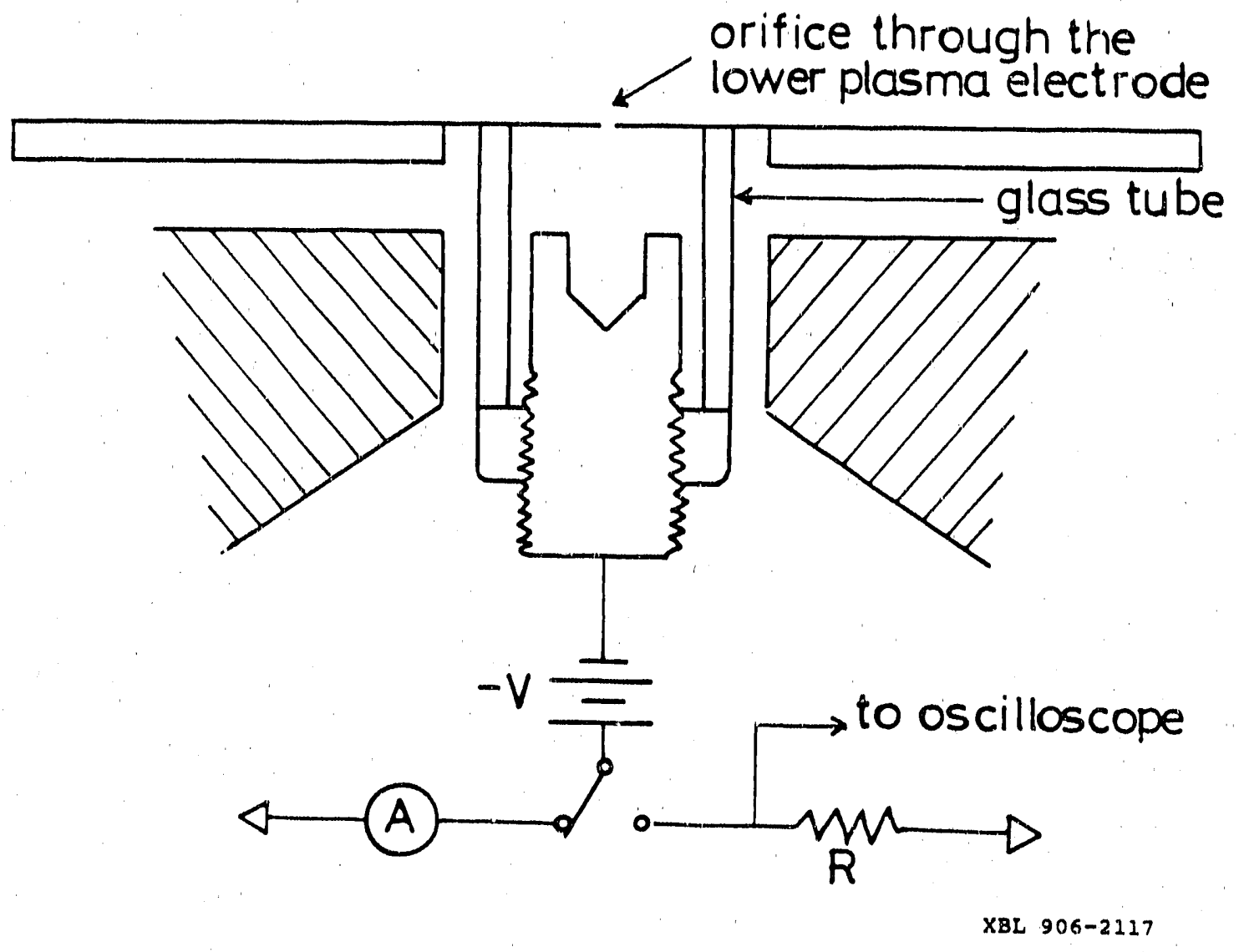

Figure 2.5: Schematic diagram of the faraday cup system used to measure the total ion flux. 
between pulses. If there were changes in ion current with time, the steady state D.C. current could not be used to calculate the average energy flux. To observe possible temporal variations in ion current, the aforementioned A.C. current measurement technique was employed.

Values measured for ion current increased with increasing Faraday cup bias voltage in the voltage range from 0 to $-200 \mathrm{~V}$. This result is not surprising as the collection efficiency of positive ions improves and stray electrons are repelled more effectively as small negative voltages are placed on a Faraday cup. The measured values reached a plateau at Faraday cup bias voltages of -200 to $-400 \mathrm{~V}$. The values measured at this plateau for ion flux agreed vithin $20 \%$ with measurements made in the AC mode. Overall measured ion current values were reproducible to $\pm 10 \%$. A decrease in the measured values was noticed at bias voltages greater than $-600 \mathrm{~V}$. The origin of this decrease was not clear, possibly due to a variation in the recollection efficiency of secondary electrons. The values measured at a Faraday cup bias voltage of $-400 \mathrm{~V}$ were used exclusively for all the ion energy flux values presented in this paper. Since ion flux measurements are discussed as being an upper limit on the true values, using the values measured at $-400 \mathrm{~V}$, in the plateau region, seems most appropriate.

Fig. 2.6 shows typical oscilloscope traces of the current-induced voltage signal across a $1 \mathrm{M} \Omega$ resistance, connected between the ion collector and ground. The lower trace in Fig. 2.6 shows the signal which was obtained under no direct ion flow to the collector. Due to capacitive coupling between the lower electrode and the ion collector, the signal exhibits the same pulse pattern as the applied voltage. When the direct pathway of ions to the collector is opened, the increase of the observed current due to the impinging ions results in an upward shift of the oscilloscope trace as shown in Fig. 2.6. It can be seen that the two traces in Fig. 2.6. are virtually identical in shape, but simply shifted in the vertical direction. This fact indicates that there is no observable temporal variation of ion flux, even at the pulsed biased electrode. As a result, the calculation of the average ion energy flux is simplified. It is given by the product of the steady state D.C. current density and the time-averaged potential drop between the lower electrode and the plasma bulk. 


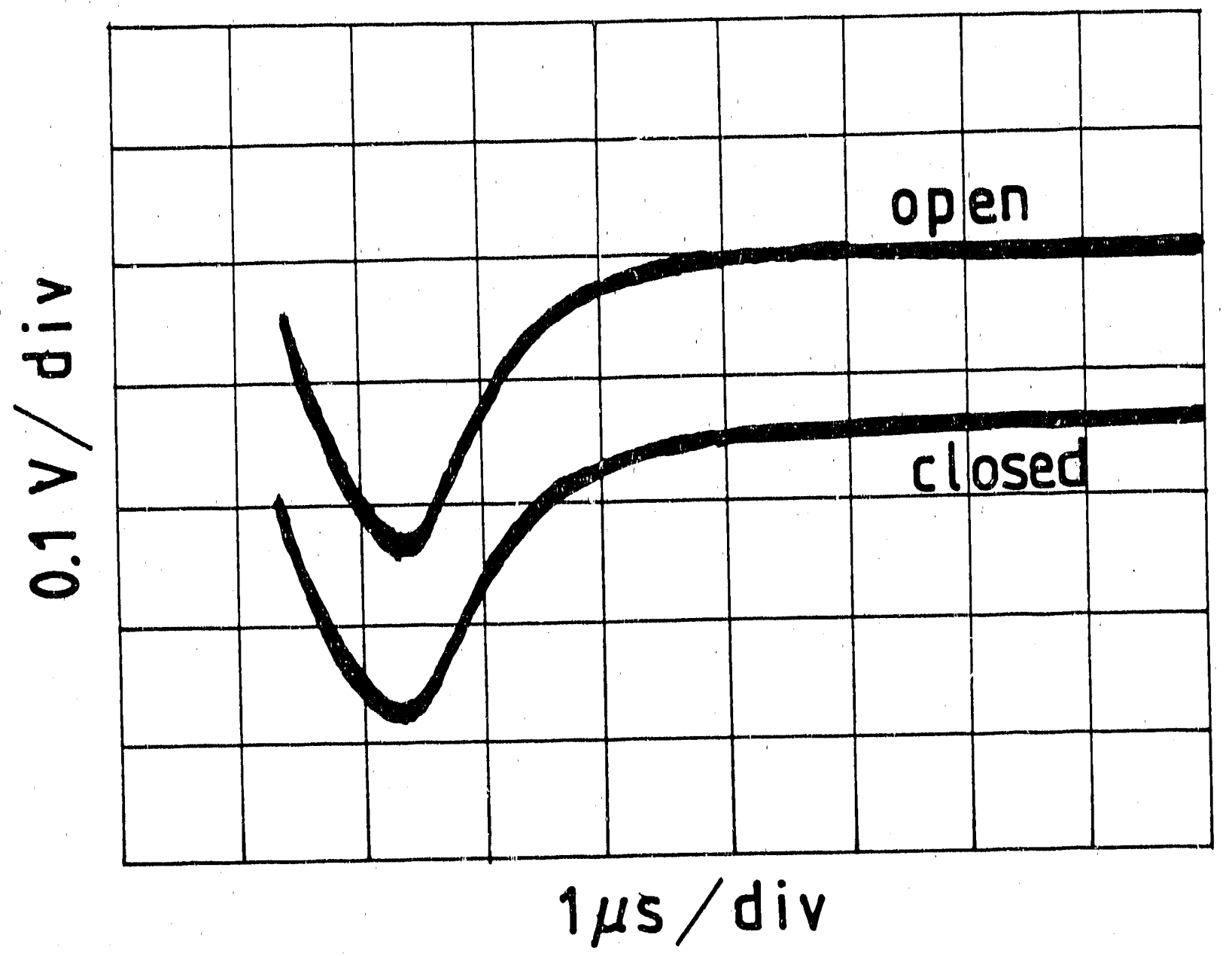

XEL $906-2118$

Figure 2.6: Oscilloscope trace of the measured current during the pulsed bias conditio. The equivalent shape of the background level and the total current level, measured with the ion orifice open, indicates that no measurable temporal variation in ion flux exists. 


\subsubsection{Pulsed Biasing}

A method to control the in energy flux separately from the neutral radical flux was required so that studies of the variations in film properties caused by independent variations in ion energy flux could be carried out. Pulses of large negative voltages $(-20$ to $-1100 \mathrm{~V})$ were applied to the otherwise grounded electrode in order to extract positive ions from the plasma. Ion energy was controlled through variation of pulse height. The flux of the high energy ions was regulated by means of varying the pulse frequency. The pulse width was small (ca. $2 \mu \mathrm{s}$ ), so that the duty cycle of the method was only a few percent. The energies and trajectories of radicals are not affected by potentials applied. With such a small duty cycle, the radical densities should also remain relatively constant.

Pulsed biasing is a variation on the method of plasma source ion implantation developed by Conrad and coworkers[8] and modeled by Lieberman[9]. In the present work, we are using much smaller pulse heights than are typically employed in plasma source ion implantation, where pulse heights of tens of $\mathrm{kV}$ are common. Thus we are bombarding the surface with ions of much lower energy and not implanting them in the traditional sense.

The high voltage pulse generator system used for the pulsed biasing of the nonpowered electrode was constructed in this laboratory, and is capable of producing negative pulses of more than $1 \mathrm{kV}$ with a pulse width of about $2 \mu \mathrm{s}$, at frequencies less than $30 \mathrm{kHz}$. The frequency limit was increased up to $100 \mathrm{kHz}$ for negative voltages less than $80 \mathrm{~V}$, by making use of only the trigger pulse generator in this systern.

\subsection{Mechanical Properties}

\subsubsection{Knoop Hardness}

The hardness of a material is a measure of its resistance to deformation. It is, therefore, one of the most important properties of a material to be used as a wear resistant coating. The relative hardness of two materials can be easily determined. The harder material will more readily sciatch the softer one. This is the basis of the 
oldest hardness measurement, where Mohs[10] ranked materials on a relative hardness scale ranging from one (scratch hardness of talc) to ten (scratch hardness of diamond).

More quantitative tests of hardness have been developed, based on measurements of the extent of deformation which occurs when a very hard (usually diamond) indenter of a known geometry is pressed with a known load into the material in question. The extent of deformation is determined from the projected area of the indentation. One such test is the Knoop hardness test. Developed by the National Bureau of Standards in 1939, the Knoop hardness test is most useful when determining the hardness of films with thicknesses of $1 \mu \mathrm{m}$ or greater.

The Knoop indenter (Fig. 2.7) is a highly polished rhombic based pyramidal diamond with an included transverse angle of $130^{\circ} 0^{\prime}$ and an included longitudinal angle of $170^{\circ} 30^{\prime}$. The advantage of this indenter geometry is that the depth of indentation is rnuch smaller than the length of the longest diagonal (ratio is about 1:30). This characteristic makes it especially suited for hardness measurements on thin films. The Knoop indenter is typically used with loads ranging from $1 \mathrm{gf}$ (gram force) to 1000 gf, and produces a diamond shaped indentation. The ratio of the length of the long diagonal of the indentation to the short one is about 7 to 1 . An optical microscope is generally used to measure the longer diagonal of the indentation, although electron microscopes can be used for very small indentation lengths where the diagonal length is less than $10 \mu \mathrm{m}$.

The Knoop hardness number $(H K)$ is calculated as the ratio of the applied load over the projected area of the indentation, using the relation:

$$
H K=\frac{P}{A}=\frac{P}{C L^{2}}
$$

where $P$ is the applied load in kgf; $A$ is the projected area of indentation in $\mathrm{mm}^{2} ; L$ is the measured length of the longer diagonal in $\mathrm{mm}$; and $C(0.07028)$ is a constant for the indenter relating the projected area to the square of the length of the longer diagonal[7].

The Knoop hardness of the films was measured with a Buehler Micromet microhardness tester with loads from 20 to 5 grams for films thicker than $1 \mu \mathrm{m}$. The indentation diagonal lengths were measured by optical microscopy. Indentation di- 


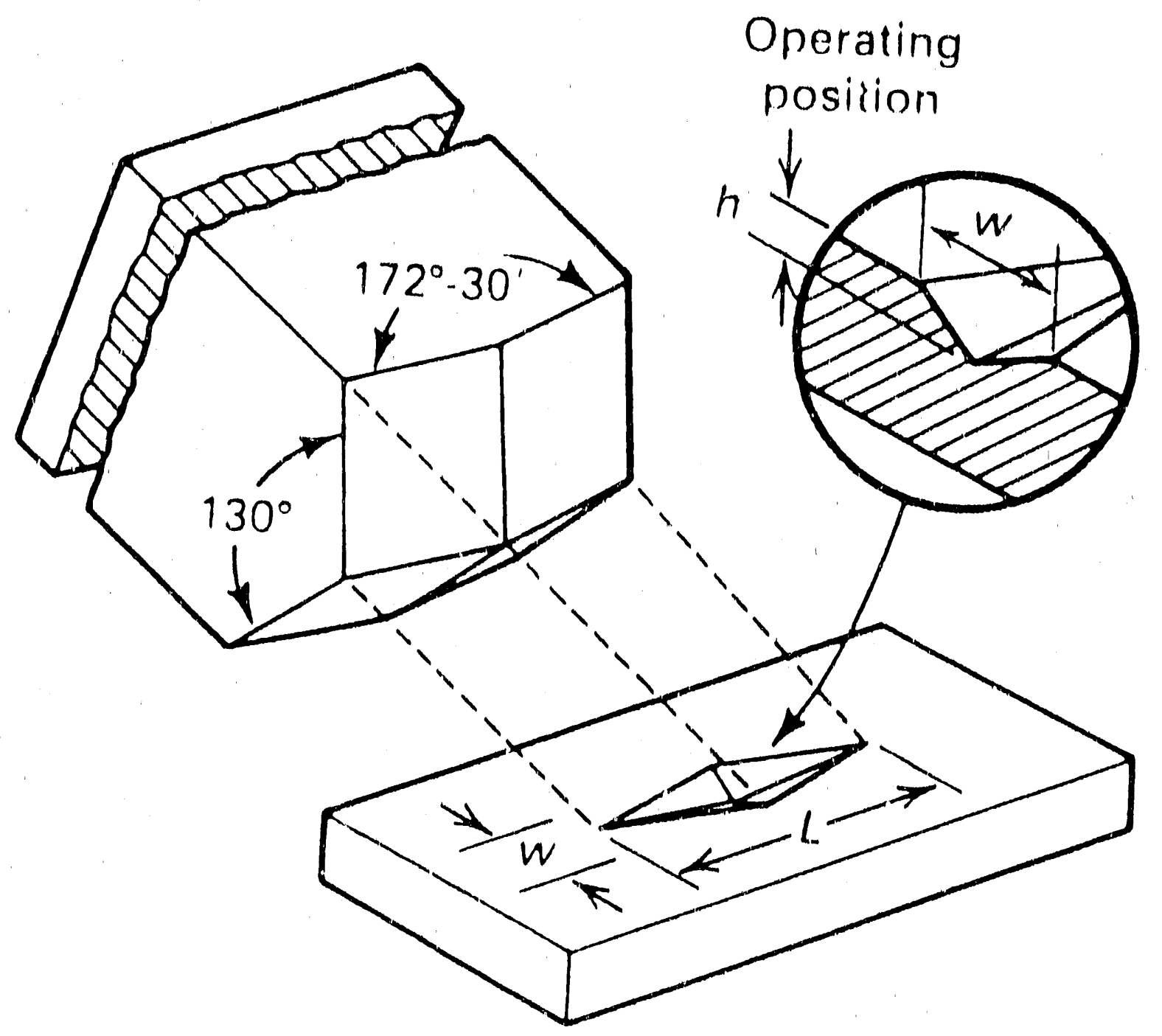

XBL 906-2119

Figure 2.7: Schematic of Knoop diamond indenter and the resultant indentation geometry. 
agonal lengths of less than $10 \mu \mathrm{m}$ were measured by Scanning Electron Microscopy (SEM).

\subsubsection{Thickness and Stress}

The thickness of deposited fllrns was measured using a Clevite Surfanalyser 150 profllometer, with a diamond tip scanning across the step between the film and an urea which had been masked during deposition. The profilometer was also used to measure the degree of bending of the silicon substrate, induced by the stress in the deposited films. The compressive stress within the deposited film was calculated using the conventional formula[11].

$$
S=\frac{4 E D^{2} \delta}{3 L^{2}(1-u) T}
$$

where $S$ is the stress; $\delta$ is the deflection of the bending; $E$ is the Young's modulus for the substrate; $D$ is the thickness of the film; $L$ is the length of scanned substrate segment; $\nu$ is the Poisson's coefficient of the substrate; and $T$ is the thickness of the film.

Film density was determined from the measured mass of the film and its volume as calculated from the measured thickness and area.

\subsubsection{Scratch Adhesion Test}

Adhesion can be defined as the bonding of two different materials at an interface. Adhesive strength is the amount of energy required to pull apart the two surfaces at the interface. Although widely varying magnitudes of adhesive strength are often and easily sensed in everyday life, a quantitative measurement of this property is extremely elusive. In order to quantitatively measure adhesion, the real area of contact of the interface musı be known. Also, the energy dissipated in the cleaving of the materials must be separable from all other avenues of energy dissipation such as dislocation formation, surface reconstruction, and surface chemical reactions. Neither of these two conditions have been met in current adhesion measuring tests. It seems unlikely that these conditions can be met, strictly. As a result, we are presently unable to measure the strength of adhesion of a film to its substrate quantitatively. 
However, there are tests which can evaluate the adhesive characteristics of a film to its substrate. These tests can provide a figure of merit for the 'scratch resistance' of a coating, a charactaristic which can reflect how well a coating might withstand delamination due to abrasion. The scratich adhesion test is such a test. As shown in Fig. 2.8, the test conslsts of intruducing stresses at the interface through the deformation which occurs in the coating when a diamond tip $(\mathrm{r}=200 \mu \mathrm{m}$, conical angle $\left.=120^{\circ}\right)$ is dragged along the surface of the coating with a known normal load. The normal load is increased until the deformation causes a flaking, cracking, or stripping off of the coating. The smallest load at which failure occurs, at the interface or in the coating, is called the critical load. The critical load is the figure of merit which can be used to compare the performances of various coatings.

In this work, scratch adhesion tests were performed on an apparatus described previously[12] as well as on a new instrument designed to perform the equivalent function in ultra high vacuum (UHV). This device, shown in Fig. 2.9, was designed and built in this laboratory. It consists of a bar onto which four tips are attached, including the Rockwell $\mathrm{C}$ tip for the scratch adhesion test, as well as Vicker's and Knoop tips for microhardness measurements. The bar is transferable into the UHV bell jar of a PHI 660 scanning auger system. Strain gauges, attached to the bar, measure the normal, irce as well as the perpendicular frictional force.

In conducting the scratch adhesion test measurement, the samples were raised up to contact an inverted diamond tip in the chamber. The samples were further raised up, against the force of the bending har, until the desired load was applied, as measured by the strain gauges. Then the sample was moved perpendicularly to the surface normal at a scratch velocity on the order of $10 \mathrm{~mm} / \mathrm{min}$. The resultant damage was observed by scanning electron microscopy. Scanning auger spectroscopy was used to observe whether or not substrate material was exposed during the process of scratching.

\subsection{Film Composition}

\subsubsection{Auger Electron Spectroscopy}

Auger electron spectroscopy (AES) is among the most widely used techniques 


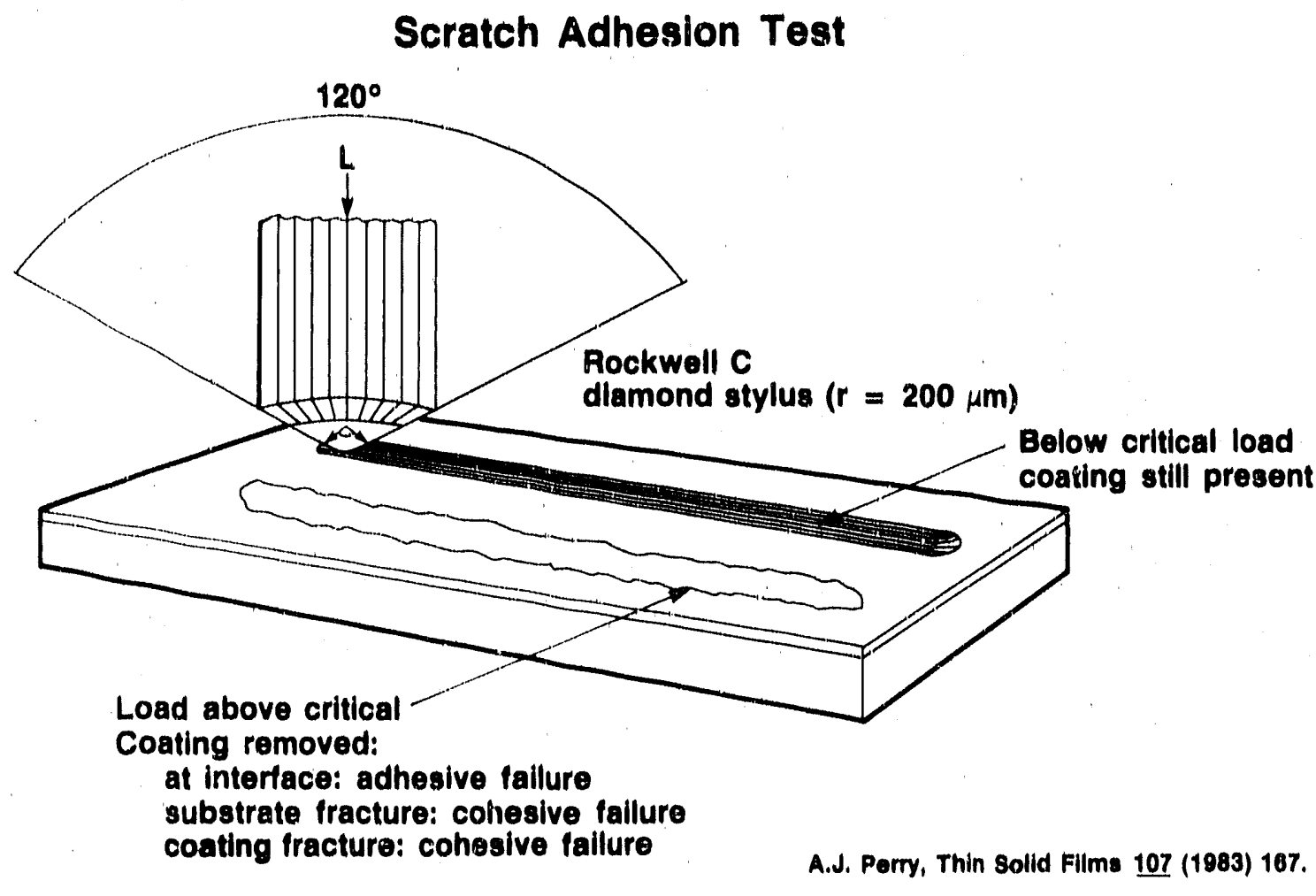

XBt. 869.8978

Figure 2.8: Schematic of the Scratch Adhesion 'Test. 


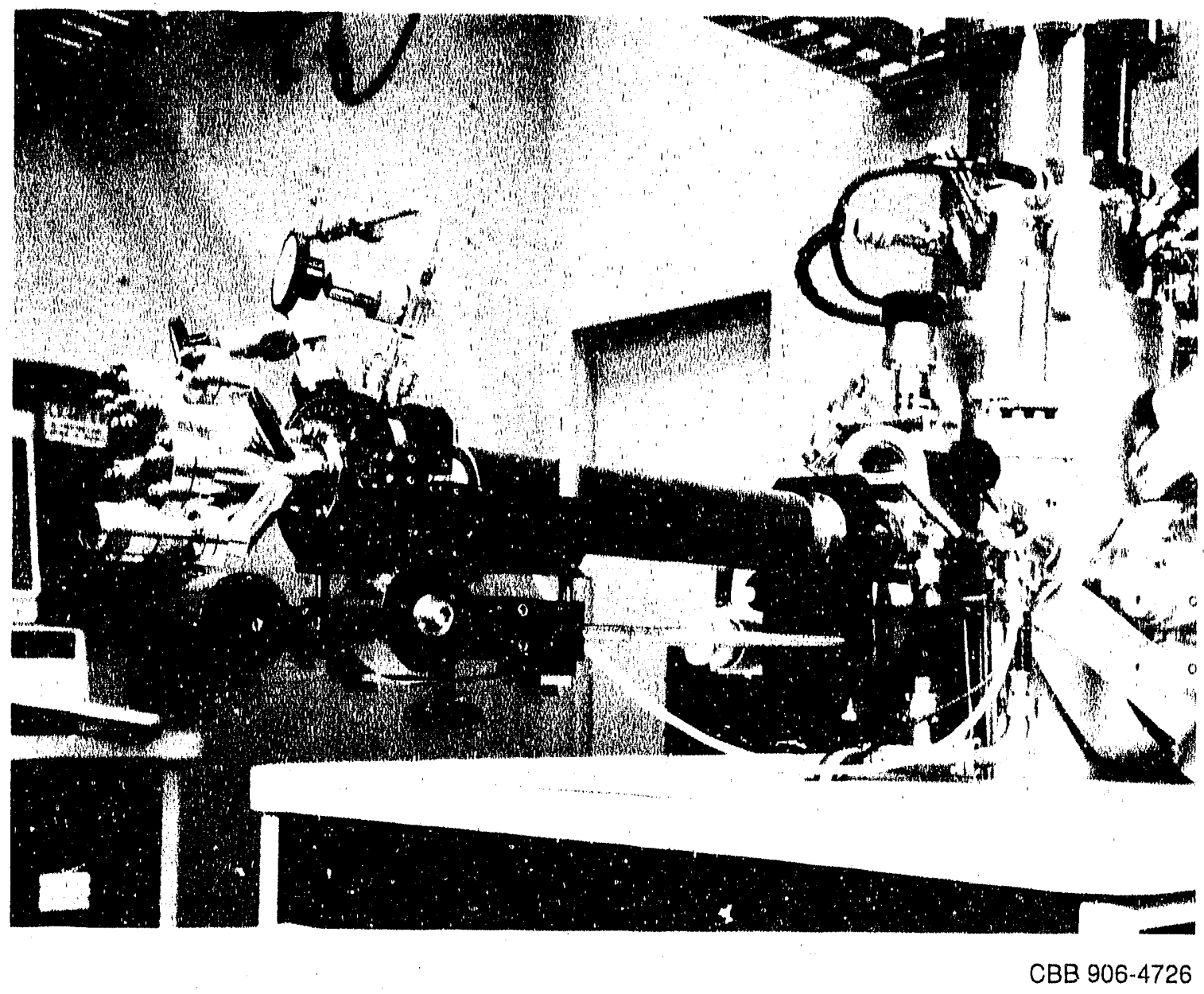

Figure 2.9: Photograph of UHV Scratch Adhesion Tester and tribometer. 
for probing surface chemistry[13]. Auger electrons are produced whenever a material is irradiated with electrons, ions, photons or neutral atoms of sufficient energy to cause ionization of the target atom through ejection of core level electrons $(\mathrm{K}, \mathrm{L}, \mathrm{M}, \ldots$ levels). The resultant ion is highly unstable and will spontaneously deexcite. This de-excitation can occur via two pathways as shown in Fig. 2.10. In one process, a higher level electron will drop down to fill the core hole, the excess energy being given off by $x$-ray radiation. If the second process occurs, namely the Auger process, the energy released by de-excitation is transferred to another electron in an outer level. This Auger electron is ejected with the characteristic kinetic energy, $E_{k e}$, where:

$$
E_{k e}=E_{K}-E_{L}-E_{L I I, L I I I}-\phi
$$

and $\phi$ is the work function of the electron analyser. The work function is the kinetic energy lost by the electron in escaping from the surface into the vacuum.

Since the energy with which an Auger electron is emitted from the surface is characteristic of the element from which it was ejected, Auger electron spectroscopy is a tool which measures surface composition. The reason that the technique is surface sensitive is because Auger electrons have a finite escape depth from the solid. Generally the energy of Auger electrons falls in the range from 20 to $2500 \mathrm{eV}$. The corresponding electron escape depths range from 2 to 10 monolayers.

Another important advantage of Auger electron spectroscopy is the lateral resolution which can be obtained. When a highly focussed electron beam is used as the excitation source, elemental composition can be obtained for sample areas as small as $0.1 \mu \mathrm{m}$ in diameter. With the advantages of surface sensitivity and good lateral resolution, Auger electron spectroscopy has proven to be a very useful tool in the study of thin films. AES is also well suited for the observation of damage which may have occurred in the process of a scratch adhesion test. Used in conjunction with ion sputtering, compositional depth profiles can be obtained, also exseedingly useful in the study of thin films.

In this study, Auger electron spectroscopy was performed using a Phi 660 Scanning Auger Spectrometer, in the same vacuum system where scratch adhesion tests could be carried out. Depth profiling was employed to monitor the resultant damage from 


\section{Excitation}
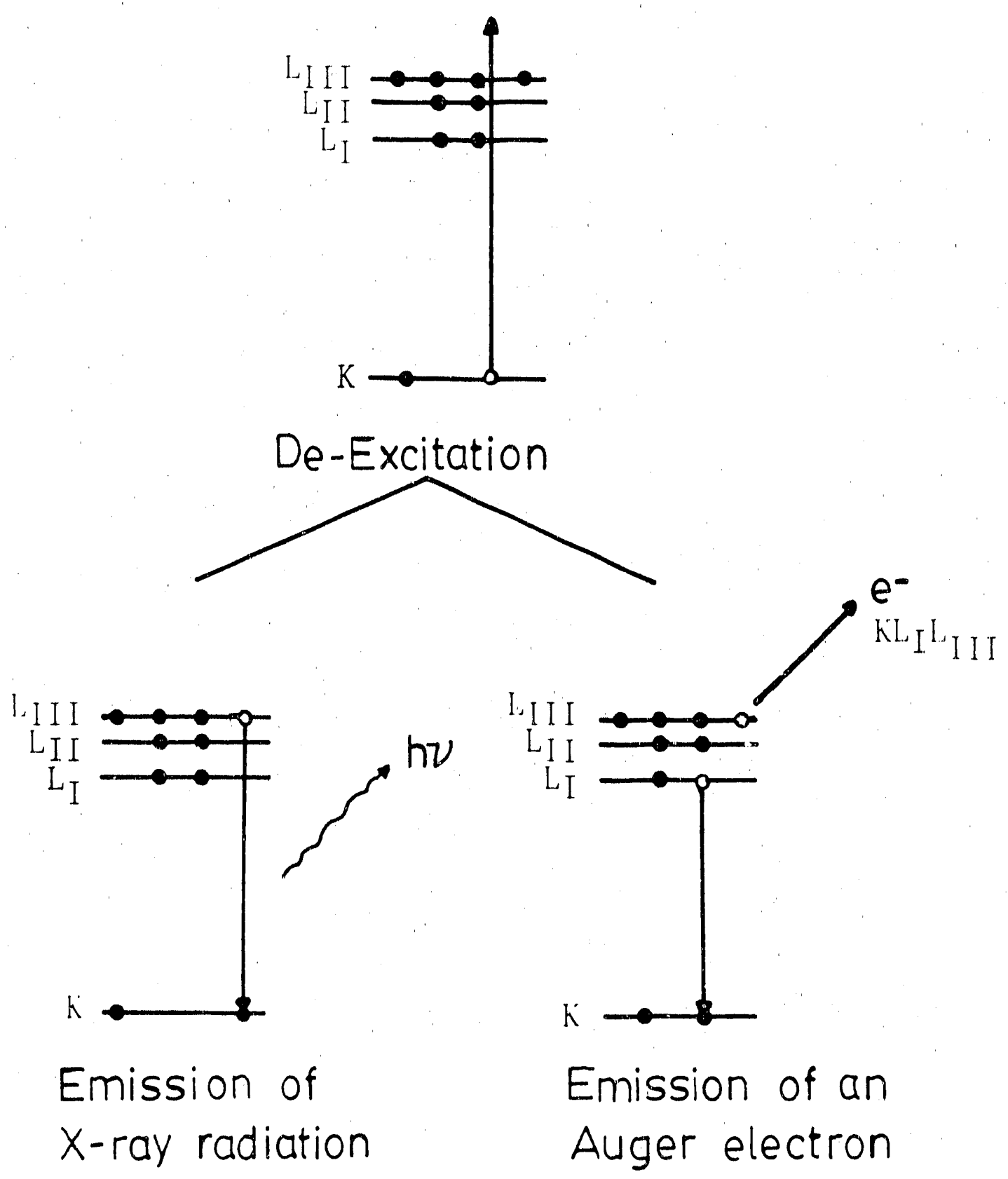

XBL 878-3631

Figure 2.10: Schematic diagram of the electronic transitions in the auger process. 
scratch adhesion tests.

\subsubsection{X-ray Photoelectron Spectroscopy}

$\mathrm{X}$-ray photoelectron spectroscopy (XPS) is another widely used surface analytical technique. While, like AES, XPS can determine elemental composition, XPS can go a step further, also determining the chemical oxidation state of the elements present.

XPS is based on the photoelectric effect, which was described in 1905[14]. A core electron is ejected from the material by an incident $\mathrm{x}$-ray photon. The electron leaves the surface with a kinetic energy $E_{k e}$ :

$$
E_{k e}=h \nu-B E+\phi
$$

where $h \nu$ is the energy of the incident photon, $B E$ is the binding energy of the ejected electron in the solid, and $\phi$ is the spectrometer work function.

The final kinetic energies of the emitted photoelectrons fall in the same range as do Auger electrons, and thus have an escape depth in the range of $2-10$ monolayers. Thus XPS is also a surface sensitive analytical technique. As mentioned, one of the important advantages of XPS is its ability to identify the chemical oxidation state of detected elements. Shifts in the binding energy of core electrons occur as a function of nearest neighbor composition, oxidation state, crystal structure, or compound type. These shifts are measurable in XPS and are called chemical shifts.

Chemical shifts result from a change in the valence shell electron density. A change in valence shell electron density will affect the nuclear attractive force on the core electrons, as the valence electrons exert a repulsive force on the core electrons. The removal of valence electron density will reduce the repulsion and result in an increased nuclear attractive force, that is, an increased binding energy. The addition of valence electron density acts in the opposite direction, reducing the binding energy. Covalent bonds can act to alter the valence electron density around an atom and lead to a chemical shift of the measured binding energy. If the chemical shift is large enough, the relative amounts of the element in question, bonded in different chemical states, can be determined by deconvolution of the spectra. Computer deconvolution of XPS data can be quite useful in many cases but must be used with caution[15]. 
Broad peaks separated by small shifts in binding energy can often not be deconvoluted uniquely. It is often useful to obtain a 'fingerprint' spectrum for at least one of the components being separated so that its binding energy is known and can be fixed in the deconvolution procedure. Interpretation of computer generated deconvolutions must be done carefully, always considering how unique and meaningful the deconvolution is.

$\mathrm{X}$-ray photoelectron spectroscopy is especially useful in the study of thin films, as the surface sensitivity of the technique can be changed. XPS surface sensitivity can be controlled by varying the takeoff angle of detection of the escaping photoelectrons. Let $\theta$ be the angle between the the surface tangent and the direction of the ejected electrons. As $\theta$ approaches zero, the distance that photoelectrons must traverse through the solid before escaping from the sample increases as $z / \sin \theta$, where $z$ is the depth below the surface of the point where the electron was emitted. If $\lambda$ is the mean free path of the electron in the material, the probability of escape decreases as $\exp (-z / \lambda \sin \theta)$. By decreasing the takeoff angle, therefore, one can make an XPS measurement increasingly surface sensitive. In this way, one can separate the surface composition of a thin film from the composition of the substrate. Variations in intensity with takeoff angle can also be used to determine the thickness of thin films on substrates of a different chemical composition.

The XPS spectra in this work were taken with $\mathrm{MgK} \alpha$ radiation of $1253.6 \mathrm{eV}$, on a Phi 5500 photoelectron spectrometer. Rotation of the specimen holder allowed the electron takeoff angle to be varied from $60^{\circ}$ to $15^{\circ}$ with respect to the surface tangent. The angle between the $x$-ray source and the emitted electrons collected by the energy analyser was fixed at $45^{\circ}$, so that the angular dependance of the photoelectric emission cross-section did not affect the angle resolved XPS spectra.

\subsubsection{Fourier Transform Infrared Spectroscopy}

Infrared spectroscopy provides valuable information about the molecular structure of a material[16, 17]. It differs from the techniques described previously in that it is a bulk technique. The frequencies of molecular vibrations generally overlap the frequencies of photons in the infrared region of the spectrum. By irradiating a 
sample with infrared light, molecular vibrations can be excited by the absorption of photons. The amount of absorption can be measured as a function of energy, and the resultant infrared spectrum of a material gives information useful in determining which functional groups are present and in what concentration. Shifts in the frequency of molecular vibrations can reveal subtle structural details. For a molecular vibration to be infrared active, the vibration must cause a change in the dipole moment of the molecule[16].

Commercial instruments are available which can detect changes in infrared intensity as a function of energy or wavelength. This can be accomplished using a dispersive instrument, or using a Fourier transform spectrometer. Dispersive instruments contain a monochromator which separates light as a function of wavelength. A mechanical slit is then used to select out the wavelength of interest which is focussed on a detector. By moving the slit with respect to the dispersed light, a range of wavelengths can be scanned in order to generate an IR absorption spectrum. In Fourier transform infrared spectroscopy, an interferometer is used to modulate the intensity of each wavelength of light at a different frequency[7]. Constructive and destructive interference between a beam passing through the sample and a reference beam, is employed in the collection of an interferogram. The interferogram must be Fourier transformed in order to generate the single beam infrared spectrum. A complete description of the details of FTIR spectroscopy is beyond the scope of this text and can be found elsewhere[17].

FTIR spectroscopy offers a number of advantages over dispersive IR spectroscopy. Spectral information from the entire wavelength range of interest is collected in a single interferogram. Such an interferogram can be collected in about one second. One can collect a number of scans and thus take advantage of signal averaging to improve upon the signal to noise ratio of the data. An additional advantage of using FTIR is that the throughput of an FTIR instrument is much greater than that of a dispersive instrument since no slits are necessary. This results in a further improvement in signal to noise characteristics.

In the case of amorphous hydrogenated carbon films, IR spectroscopy can be used to probe the various types and amounts of $\mathrm{C}-\mathrm{H}$ bonding present $[2,19]$. The 
concentrations of methyl $\left(-\mathrm{CH}_{3}\right)$ and methylene $\left(-\mathrm{CH}_{2}-\right)$ groups can be determined using Beer's law and known values for the molar extinction coefficients[20]. In the present work an FTIR spectrometer was used in its transmission mode to obtain infrared spectra of a-C:H films grown on silicon substrates.

\subsubsection{Ultraviolet/Visible Absorption Spectroscopy}

Ultraviolet/visible (UV/VIS) absorption spectroscopy can be used to determine the optical gap of materials. Thin films of a-C:H carbon are often used as protec tive coatings over optical films, thus their optical transmission characteristics are important. In this technique, the visible and ultraviolet absorption of a material is measured as a function of energy. The absorption spectrum can be used to calculate an optical gap, $E_{g}$, for a material from the intercept of a Tauc[21] plot of:

$$
(\alpha E)^{1 / 2}=\beta\left(E-E_{g}\right)
$$

where $\alpha$ is the absorption coefficient and $\beta$ is a constant for the material.

The optical gap of a-C:H films deposited under various plasma conditions has been measured previously. Hard films often demonstrate a small gap as low as 0.5

$\mathrm{eV}$ and soft films a gap as large as $4.0 \mathrm{eV}[22,23]$. It has been proposed[24] that the decrease in optical gap may originate from the presence of small $\mathrm{sp}^{2}$ bonded clusters (30- $40 \AA$ ), which may be linked together by $\mathrm{sp}^{3}$ bonded carbon to form a rigid three dimensional structure. Thus measurements of the optical gap of a-C:H films not only provide useful information for those interested in optical applications of the films, but changes in the optical gap may alșo reflect changes in the type of bonding present in the films.

In this work UV/VIS spectra were taken of a-C:H films deposited on glass slides using a Perkin Elmer Lambda 9 spectrophotometer. The optical gap was determined from the intercept of the Tauc plot[21].

\subsubsection{Secondary Ion Mass Spectroscopy}

In secondary ion mass spectroscopy (SIMS) a beam of energetic ions (1 to 20 $\mathrm{keV}$ ) is focussed onto the surface of the sample[25]. As a result of the ion-surface 
collisions, neutral and charged species are ejected from the sample in a process called sputtering. The charged particles ejected, narnely the secondary ions, can be collected and separated according to their charge to mass ratio. Using this method, a variety of information can be gained about the surface, the near surface, or the bulk of the sample. In static SIMS, the sputtering rate is maintained at a very low level, so that a complete mass spectrum can be obtained before a significant portion of the surface has been bombarded. A much greater sputtering rate can be used to determine how intensity varies as a function of depth for one or more peaks of interest. This approach is used more commonly and is referred to as dynamic SIMS.

The advantages of SIMS include very high sensitivity as well as superior lateral resolution capabilities. Sometimes structural information can also be inferred from the identity of cluster species ejected from the surface. The major disadvantage of SIMS is the presence of so-called matrix effects. The sputtering cross-section is the number of ions, of a given type, which are ejected per incident primary ion. Sputtering cross-sections are strongly matrix dependant and can vary by orders of magnitude from one ionic species to the next. As a result, preferential sputtering occurs in multicomponent systems, and surface composition can be drastically changed during the measurement. A detailed treatment of the many variables which affect secondary ion emission cross-sections can be found elsewhere[25].

ivevertheless, SIMS can be an informative tool in the area of trace analysis, and can also be used relatively easily in order to determine the relative concentrations of elements in similar matrices. Another important advantage of SIMS over AES and XPS is that it can detect hydrogen.

In this work SIMS was performed using a Phi 660 spectrometer equipped with a quadrapole mass analyser. Attempts were made to monitor the relative hydrogen concentrations of a-C:H samples synthesized under varying plasma conditions. SIMS was also employed to investigate the composition and thickness of the a- $\mathrm{C}: \mathrm{H} /$ silicon interface. 


\subsection{Film Structure}

\subsubsection{Scanning Tunneling Microscopy}

Scanning tunneling microscopy is a newly developed technique[26] which allows one to investigate the structure and morphology of a surface with atomic resolution. In this technique, conceptually depicted in Fig. 2.11, a sharp metallic tip is electrically biased with respect to the sample. The tip is scanned above the sample surface at small enough distances so that tunneling current is measurable. A feedback loop is used to adjust the tip to sample distance, $z$, in such a way as to keep the current constant. A piezoelectric manipulator is used to control the movements of the tip. As the tip is rastered across the surface the $z$ position of the manipulator is recorded as a function of rastering position. The data generated gives, to a first approximation, a topograph of the surface, called a constant current image. Unlike topographs obtained with more traditional techniques, however, an STM topograph can be recorded with atomic resolution. Thus the atomic structure at the surface can be imaged.

A second type of image can also be generated, called a constant height image. In this type of imaging, the tip position is held constant in space in the $z$ direction as the tip is rastered across the sample surface. The tunneling current is allowed to vary, as the tip to sample distance varies from position to position across the sample surface. Tunneling current is very sensitive to variations in the distance between the tip and the sample. Tunneling current, $I_{t}$ varies with separation distance, $s$, as :

$$
I_{t} \propto e^{-\phi^{1 / 2} s}
$$

where $\phi$ is the local barrier height. The local barrier height is a property clc ely related to the work function of a material; however, it is measured locally. Consuant height images will also give images with atomic resolution. They are most useful for measuring the atomic structure of very flat surfaces.

In this work, STM imaging was performed in air at room temperature using a "double-tube" type STM similar to that built by Lyding [27], and Zeglinski [28]. The STM was controlled using electronics developed at Lawrence Berkeley Laboratory, and the data were acquired using a PC compatible computer with a 12 -bit $150 \mathrm{kHz}$ 


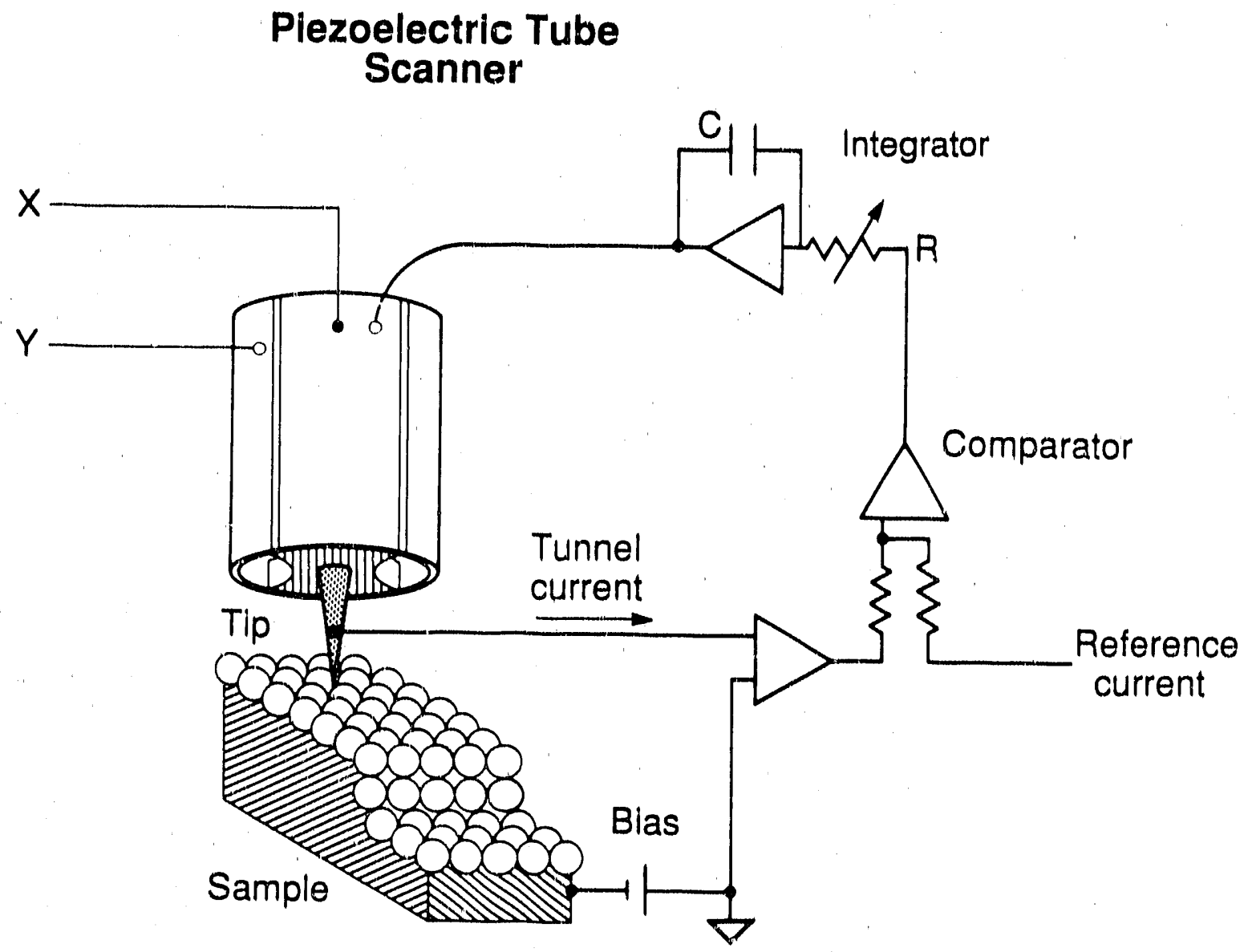

XBL 892-6157a

Figure 2.11: Conceptual depiction of the STM scanning system. 
analog to digital converter. All the images were obtained with the STM in the to. pographic (constant current) mode using tips mechanically cut from 1 mm diameter Pt-40\%Rh alloy wire. The images of films on graphite substrates were obtained using a sample bias of $-100 \mathrm{mV}$ and tunneling current of $1.0 \mathrm{nA}$. These images showed very l'ttle bias dependence between $-200 \mathrm{mV}$ and $+200 \mathrm{mV}$. Images of flims formed on the $\operatorname{Si}(100)$ substrates were obtained using a bias of $-3.0 \mathrm{~V}$ and tunneling current of 20 to $50 \mathrm{pA}$. With a-C:H on silicon substrates, the tunneling gap was unstable at higher tunneling currents, with positive sample biasing, or with negative sample biasing of less than $2.7 \mathrm{~V}$. Scan velocities from 200 to $5000 \AA / \mathrm{sec}$ were used and images were recorded with 256 points per line and 128 lines per image.

The graphite substrates were received from A. Moore of Union Carbide and were exfoliated using adhesive tape immediately before being placed on either electrode in the plasma chamber. Single crystal $\operatorname{Si}(100)$ wafers (n type, $\mathrm{P}$ doped $4-7 \Omega \mathrm{cm}$ ) were obtained from the U.C. Berkeley Microfabrication Laboratory. They were first etched in an $\mathrm{H}_{2} \mathrm{SO}_{4}: \mathrm{H}_{2} \mathrm{O}_{2}(7: 3)$ bath and then in a $10 \% \mathrm{HF}$ bath. Finally, the back sides of the wafers were coated with $0.5 \mu \mathrm{m}$ of $\mathrm{Au}$ to provide a good electrical contact. The wafers were then cleaved into $1 \mathrm{~cm}$ by $1 \mathrm{~cm}$ fragments which were then placed on the electrodes and used as substrates. The plasma chamber was mechanically pumped down to a pressure of less than $10 \mathrm{mTorr}$, then methane was admitted at $8 \mathrm{~cm}^{3} / \mathrm{min}$ to reach a preignition pressure of $65 \mathrm{~m}$ Torr. The plasma was ignited for short $(5 * 30$ sec.) deposition tines to complete the initial stages of growth. The methane flow was then stopped and the chamber pressure increased to atmospheric with Ar gas. The samples were then removed from the electrodes and transferred through air to the STM, where they were finally imaged in air.

\subsubsection{High Resolution Electron Energy Juss Spectroscopy}

High resolution electron energy loss spectroscopy (HREELS) is a useful technique for determining the structure of molecules at the surface. Like infrared spectroscopy, it is a vibrational spectroscopy. However, it has the advantage of being surface sensitive. In this technique, a monoenergetic beam of electrons is focussed on the sample surface. The electrons interact with the molecules on the surface, and can lose energy 
by vibrationally exciting the surface molecules. The scattered electron intensity is detected as a function of energy, and the resultant HREELS spectrum gives peaks at electron energy losses corresponding to the vibrational modes of the molecules at the surface. The technique was used only sparingly in this work so the reader is referred to several excellent books which have been written on the subject for further descriptions and explanations $[29,30]$.

In this work high resolution electron energy loss spectroscopy was carried out with a view towards chemically distinguishing the low coverage a- $\mathrm{C}: \mathrm{H}$ film from the graphite blank and perhaps gaining information concerning the type of C-H bonding present in the films. The sample of a- $\mathrm{C}: \mathrm{H}$ on graphite was prepared identically to those prepared for use in the STM studies, with a deposition time of 10 seconds. The graphite blank received identical treatment excluding plasma ignition. The samples were then transferred through air to an ion pumped UHV chamber which attained a base pressure of $7.5 \times 10^{-11}$ Torr. A HREELS spectrometer similar to that of Froitzheim et al [31] was used with an incident electron energy of $5 \mathrm{eV}$. The scattering intensities for specular reflection were $1.2 \times 10^{4}$ counts/second for the graphite blank and $0.8 \times 10^{3}$ counts $/$ second for the a-C:H film. The energy resolution (FWHM) for the graphite blank was $75 \mathrm{~cm}^{-1}$ and $90 \mathrm{~cm}^{-1}$ for the a-C:H film.

\subsubsection{Raman Spectroscopy}

Similarly to the way knowledge can be gained about solids by measuring the energy losses of scattered electrons using HREELS, the energy losses of photons scattered off solids can also be extremely informative, as measured in Raman spectroscopy. When a beam of light passes through a nonabsorbing medium, the intensity of the transmitted beam decreases measurably. Most of this intensity or energy is not lost into the heating of the sample, but rather is lost by the scattering of light into the space surrounding the sample[7].

Photons can be scattered in a number of ways. First of all, they can be scattered elastically, as is the case in Raleigh scattering. Here, the photon is absorbed by the solid, leaving the solid in an excited virtual state, at an energy of $h \nu_{o}$ above the ground state. Relaxation of the solid into the original state results in the emission 
of a photon of the same energy as the incident photon, hwo. Occurring with a much smaller probability are inclastic scattering events, of which there are two types. In the first type of inelastic scattering, namely Stokes scattering, the solid relaxes from the virtual state at $h \nu_{0}$ into a vibrationally excited state, at energy $h \nu_{n}$, emitting a photon with the energy $h\left(\nu_{0}-\nu_{n}\right)$. The normal method of Raman spectroscopy Involves the measurement of the intensity of this type of scattered radiation. If the incident photon is absorbed by a molecule already in an excited state, at $h \nu_{n}$, and relaxation subsequently ocrurs down to the ground state, the scattered photon is emitted with the energy $h\left(\nu_{0}+\nu_{n}\right)$. This type of scattering event is called anti-Stokes scattering and occurs with a probability even smaller than that of the Stokes process.

By collecting and measuring the energy and intensity of the scattered radiation, one can gain information concerning the concentrations of molecular species with a Raman active mode. For a vibrational mode to be Raman active, it must alter the polarizability of the molecule. This selection rule is different from the selection rule which determines which modes are infrared active, so that the techniques of Raman and FTIR spectroscopy often give complimentary information. Another reason why Raman spectroscopy can be very informative is that the energy and intensity of the Raman signal is extremely sensitive to the molecular environment of the active species of interest.

Rarnan spectroscopy is one of the few techniques sensitive to changes in the type of bonding present in carbon solids. XPS and AES, for example, show only subtle changes in the signal obtained from diamond, as compared to that from graphite. Using Raman spectroscopy, however, the two materials are clearly distinguishable. The first order Raman spectrum of diamond consists of a single line at $1332 \mathrm{~cm}^{-1}$. The corresponding spectrum of large single crystal graphite also consists of a single intense line, the $G$ line, at about $1580 \mathrm{~cm}^{-1}$ [32].

Raman spectroscopy is also very sensitive to changes in the translational symmetry and crystallinity of carbon compounds. The Raman spectrum of small graphitic. crystals (basal dimensions $\leq 200 \AA$ ) is different from that of a single crystal, as a new line appears in the spectrum of polycrystalline graphite at about $1355 \mathrm{~cm}^{-1}[32]$, called the $D$ line or the disorder line. Changes also occur in the appearance of the $G$ 
line, which have been explained by a frequency shift[33] and by the appearance of a new line[34]. These new features are Raman inactive in the single crystal graphite. In microcrystalline graphite, however, the Raman selection rule breaks down due to the loss of translational symmetry (long range order), and then otherwise forbidden transitions can contribute to the Raman spectrum[35].

The Raman spectrum of a-C:H has also been investigated and, at first glance, appears similar to that from polycrystalline graphite. Careful studies of the resonant Raman spectra of a-C:H were made by Ramsteiner and Wagner[36], who concluded that the behaviour observed was consistent with a structure for hard $\mathrm{a}-\mathrm{C}: \mathrm{H}$ of $\mathrm{sp}^{2}$ clusters interconnected by $\mathrm{sp}^{3}$ sites.

In this work, Raman spectra were measured using a single-morlochromator spectrometer equipped with an imaging photomultiplier. The system has been described previously[37]. 


\section{Bibliography}

[1] H.S. Butler and G.S. Kino, Phys. Fl. 61346 (1963).

[2] M.A. Lieberman, IEE Trans. Plasma Sci. 16, 638 (1988).

[3] R. Avni, U. Carmi, A. Inspector, and R. Rosenthal, Thin Solid Films 118, 231 (1984).

[4] B.E. Cherrington, Plasma Chem. Proc. 2, 113 (1982).

[5] K. Tachibana, M. Nishida, H. Harima, and Y. Urano, J. Phys. D 17, 1727 (1984).

[6] K. Kobayashi, N. Mutsukura, and Y. Machi, J. Appl. Phys. 59, 910 (1986).

[7] Metals Handbook, 9th edition, American Society for Metals, 1986.

[8] J.R. Conrad, J.L. Radke, R.A. Dodd, F.J. Worzala, and N.G. Tran, J. Appl. Phys. 62, 4591 (1987).

[9] M.A. Lieberman, J. Appl, Phys. 66, 2926 (1989).

[10] F. Mohs, Grundries der Mineralogie. Dresden, (1822).

[11] A. Brenner and S. Senderoff, J. Res. Natl. Bur. Stand. 42, 105 (1949).

[12] M.R. Hilton, G.J. Vandentop, M. Salmeron, and G.A. Somorjai, Thin Solid Films $154,377(1987)$.

[13] D. Briggs and M.P. Seah, Ed, Practical Surface Analysis By Auger and X-Ray Photoelectron Spectroscopy, John Wiley and Sons, 1983.

[14] A. Einstein, Ann. Physik 17, 132 (1905). 
[15] C.J. Powell and M.P. Seah, J. Vac. Sci. Tech. A 8, 735 (1990).

[16] P.C. Painter, M.M. Coleman, and J.L. Koenig, The Theory of Vibrational Spectroscopy and Its Application to Polymeric Materials, John Wiley and Sons, 1982.

[17] P.R. Griffiths, Chemical Infrared Fourier Transform Spectro:copy, John Wiley and Sons, 1975.

[18] P. Couderc and Y. Catherine, Thin Solid Films 146, 93 (1987).

[19] B. Dischler, A. Bubenzer, and P. Koidl, Solid State Commun. 48, 105 (1983).

[20] J.M. Tibbitt, M. Shen, and A.T. Bell, J. Macromol. Sci. Chem. A 10, 1623 (1984).

[21] U.J. Ta'sc, R. Grigonovici, and A. Vancu, Phys. Status Solidi 15, 627 (1966).

[22] J. Robertson, Adv. Phys. 35, 317 (1986).

[23] J. Mort and F. Jansen in Plasma Deposited Thin Films, (CRC, Baco Raton, 1986).

[24] M.A. Tamor and C.H. Wu, J. Appl. Phys. 67, 1007 (1990).

[25] A. Benninghoven et al., Ed., Secondary Ion Mass Spectrometry - SIMS II, Springer-Verlag, 1979.

[26] G. Binnig, H. Rohrer, Ch. Gerber, and E. Weibel, Phys. Rev. Lett. 49, 57 (1982).

[27] J.W. Lyding, S. Skala, R. Brockenbrough, J.S. Hubacek, and G. Gammie, J. Microscopy 152, 371 (1988).

[28] D.M. Zeglinski, D.F. Ogletree, T.P. Beebe, Jr., R.Q. Hwang, G.A. Somorjai, and M.B. Salmeron, submitted to Rev. Sci. Instrum.

[29] H. Ibach and D.L. Mills, Electron Energy Loss Spectroscopy and Surface Vibrations, Academic, New York, 1982.

[30] R.F. Willis, Ed.; Vibrational Spectroscopy of Adsorbates, Springer-Verlag, New York, 1980. 
[31] H. Froitzheim, H. Ibach, and S. Lewald, Rev. Sci. Instrum. 46, 1325 (1975).

[32] R.O. Dillon, J.A. Woolam, and V. Katkanant, Phys. Rev. B. 29, 3482 (1984).

[33] F. Tuinstra and J.L. Koenig, J. Chem. Phys. 53, 1126 (1970).

[34] H. Maeta and Y. Sato, Solid State Commun. 23, 23 (1977).

[35] R. Al-Jishi and G. Dresselhaus, Phys. Rev. B. 26, 4514 (1982).

[36] M. Ramsteiner and J. Wagner, Appl. Phys. Lett. 51, 1355 (1987).

[37] D.K. Veirs, V.K.F. Chia, and G.M. Rosenblatt, Applied Optics 26, 3530 (1987). 


\section{Chapter 3}

\section{a-C:H Film Properties}

\subsection{Introduction}

a-C:H films were deposited from methane using an rf plasma system under the deposition conditions described in section 2.1.2. Many investigators have studied the properties of a-C:H films and how they change with deposition conditions[1-6]. The deposition conditions for the initial characterization work described in this chapter were chosen to match those used in the past to synthesize a-C:H films with desirable properties[1]. The deposition system used was a parallel plate electrode system. With such a system, substrates can be placed either at the rf powered electrode, or the grounded electrode. It was immediately obvious that the properties of the films depended significantly on the choice of electrode at which they were grown. Films deposited at the grounded electrode were light in color and could be easily removed from the silicon substrates by rubbing them with a tissue. In contrast, films formed at the powered electrode were much darker in color, and could not be scratched off, even with a steel scribe.

Most of the previous work on a-C: $\mathrm{H}$ films has concentrated on the properties of films grown at the powered electrode, and ignored the softer films formed at the grounded electrode. In this work, the dependence of film properties on electrode position was investigated more carefully in hopes of gaining a better understanding of the origin of these differences.

This chapter gives the results of studies investigating the differences in the properties of films formed at the powered electrode as compared to films formed at the grounded electrode. 


\subsection{Growth Rate}

Deposition rate in terms of the increase in thickness per unit deposition time is given in Fig. 3.1 as a function of $\mathrm{rf}$ power. For our standard deposition conditions, with an rf power of $50 \mathrm{~W}$, deposition rates were measured as $140 \AA / \mathrm{min}$ on the powered electrode and $95 \AA /$ min on the grounded electrode. As will be shown in the next section, the density of the films produced on the powered electrode is greater than the density of films formed on the grounded electrode by about $30 \%$, which makes the mass deposition rate at the powered electrode twice as large as that at the grounded electrode. As can be seen in Fig. 3.1, the deposition rate is not a linear function of rf power and tends to level off after $50 \mathrm{~W}$. It should be noted that the measured deposition rate did not depend on whether the upper or lower electrode was powered. The difference in deposition rates between the powered and grounded electrodes, as shown in Fig. 3.1, is therefore not due to a gas flow effect, even though the methane was flowing out of the upper electrode, making the system somewhat asymmetric.

\subsection{Mechanical Properties}

The Knoop hardness values for the films formed on the powered electrode (PE, 34 $\mathrm{GPa}=3468 \mathrm{~kg} / \mathrm{mm}^{2}$ ) are about fifteen times that of films formed on the grounded electrode $\left(\mathrm{GE}, 2 \mathrm{GPa}=0.24 \mathrm{~kg} / \mathrm{mm}^{2}\right)$, and nearly one third that of diamond, as shown in Table 3.2. SEM images showed that films formed on the grounded electrode were much more susceptible to damage by scratch adhesion testing than were films formed on the powered electrode. Powered electrode films were not noticeably damaged by an $830 \mathrm{~g}$ scratch adhesion test, as observed by SEM (Fig. 3.3). Films formed on the grounded electrode were severely damaged by a scratch adhesion test with a load of $830 \mathrm{~g}$, and damage was noticeable by SEM at load values as low as 32 g. Relatively large areas ( $1 \mathrm{~mm}$ diameter), centered on the scratch tracks, were depth profiled and AES was performed both in the track and away from the track. Fig. 3.4 shows the $\mathrm{Si}$ and $\mathrm{C}$ AFS signal intensities as a function of depth, demonstrating the relative intensities of the silicon and carbon auger intensity in and away from the 


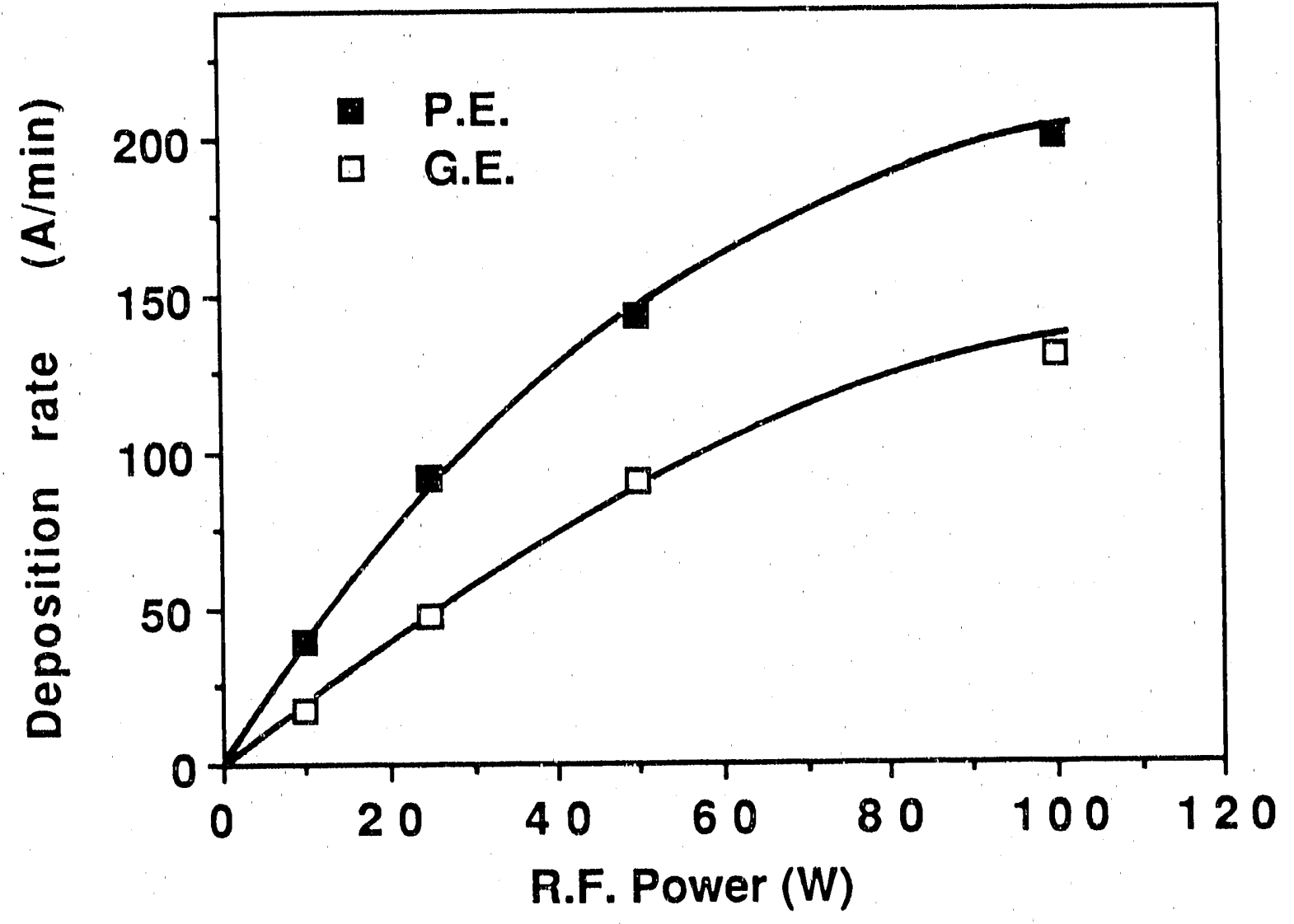

XBL $896-2523$

Figure 3.1: Deposition rate vs if power for the powered and grounded electrodes. Data points were taken for the powered and grounded electrodes in both upper and lower positions in the deposition apparatus to demonstrate the symmetry of the deposition rate with respect to gas flow. 


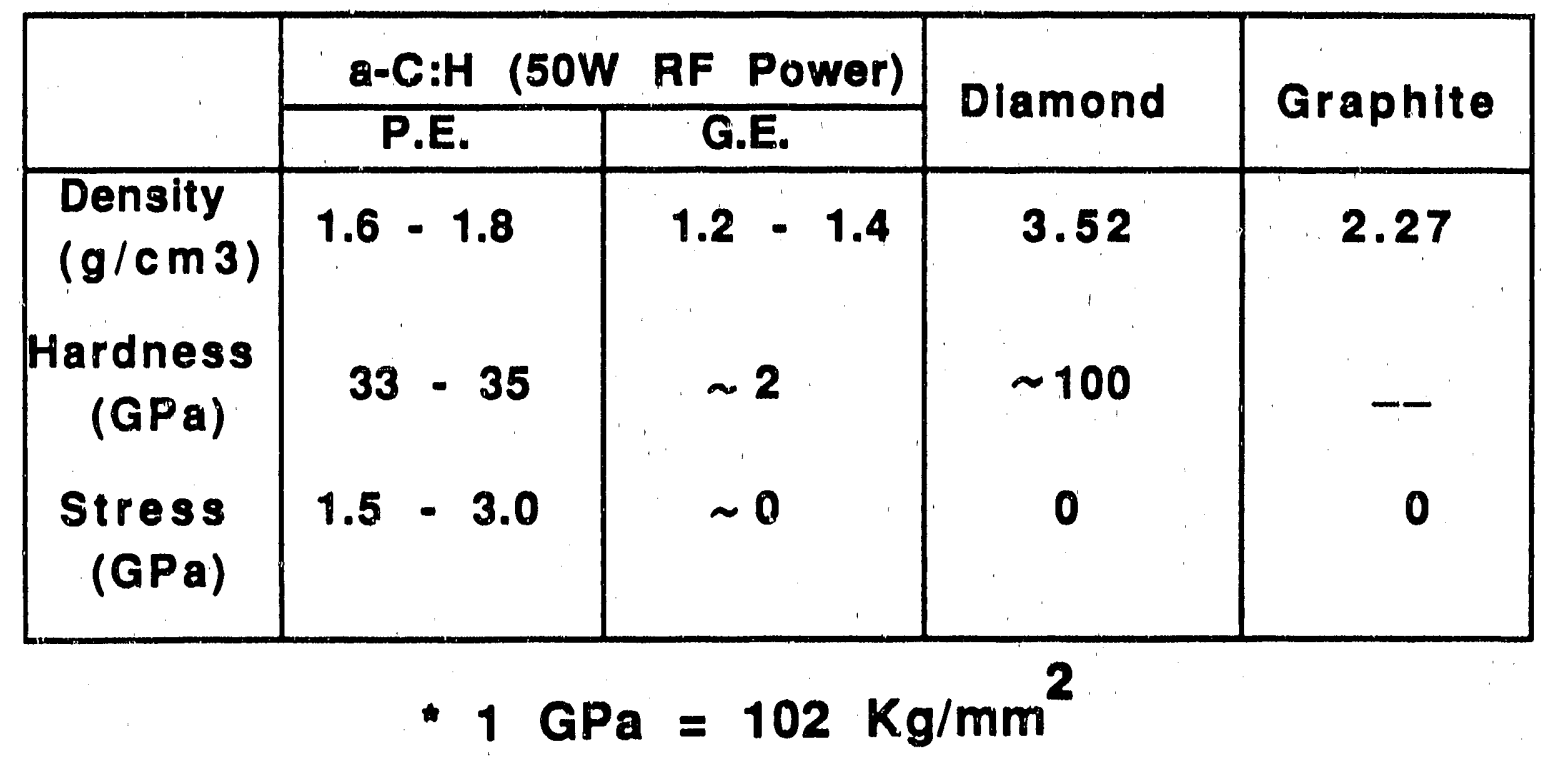

XBL 896-2531

Figure 3.2: Summary of the mechanical properties of a-C:H films, diamond, and graphite, where $1 \mathrm{GPa}=102 \mathrm{~kg} / \mathrm{mm}^{2}$. 


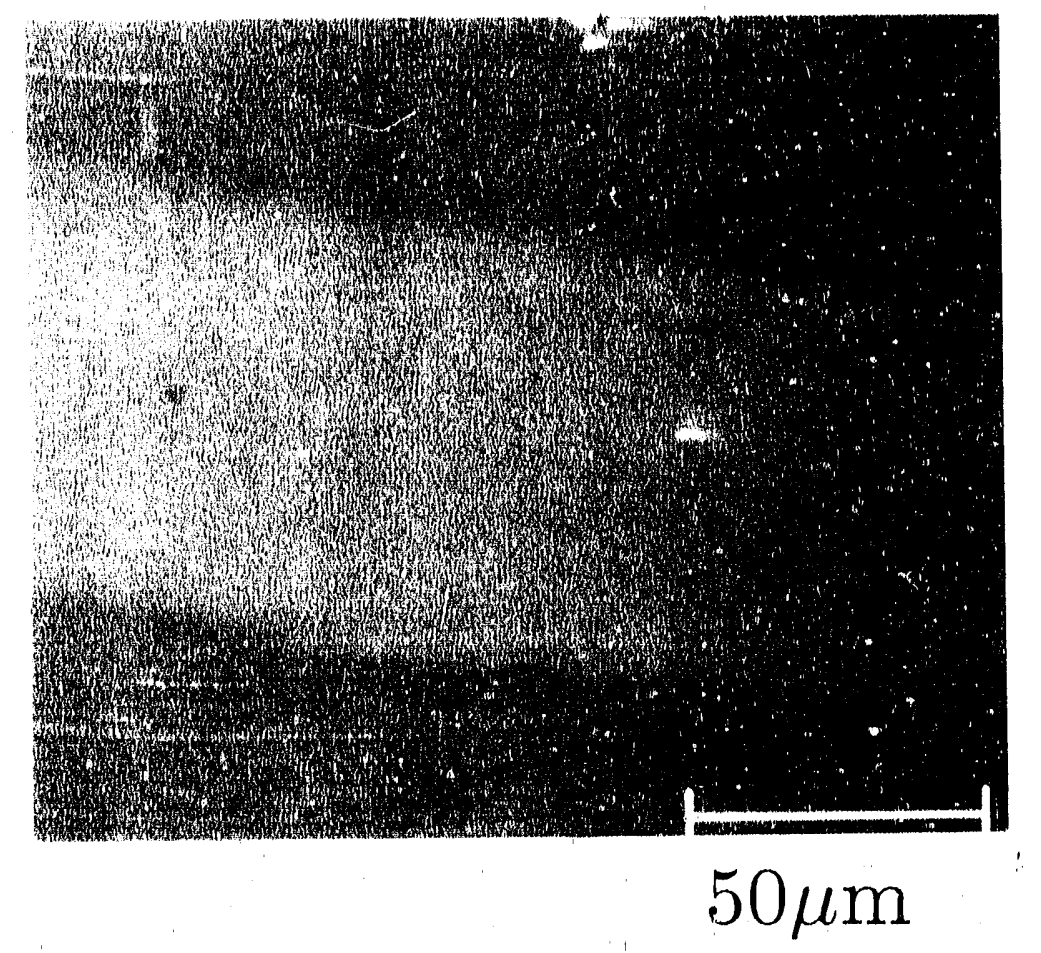

\section{powered \\ electrode}

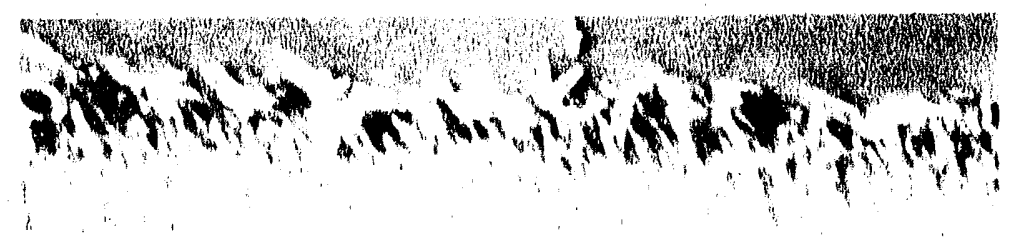

\section{grounded}

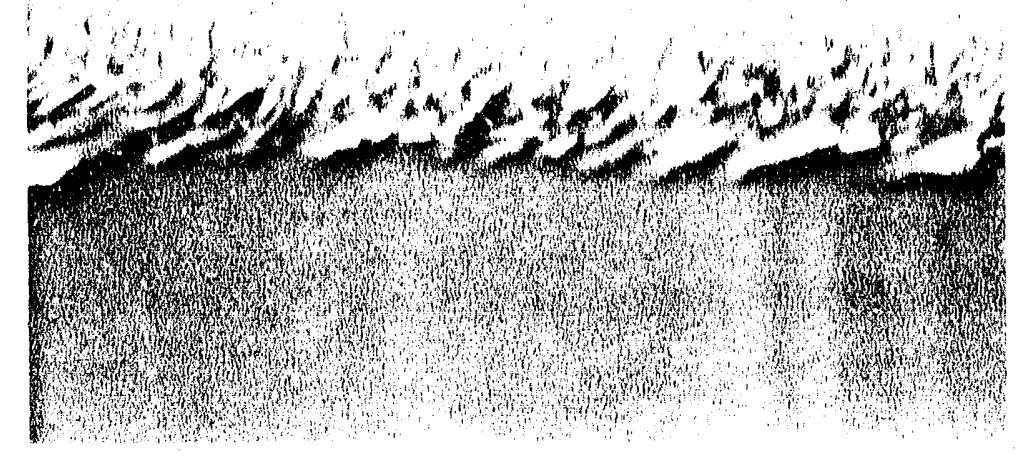

electrode

XBB 897-5454

Figure 3.3: Secondary clectron microscope images of scratch tracks made with a Rockwell ( diamond tip at a load of $830 \mathrm{~g}$, for films formed on the powered and the grounded dectrodes. Little damage is apparent on the hard films formed on the powered electrode, while the soft film formed at the grounded electrode has suffered noticeable damage. 


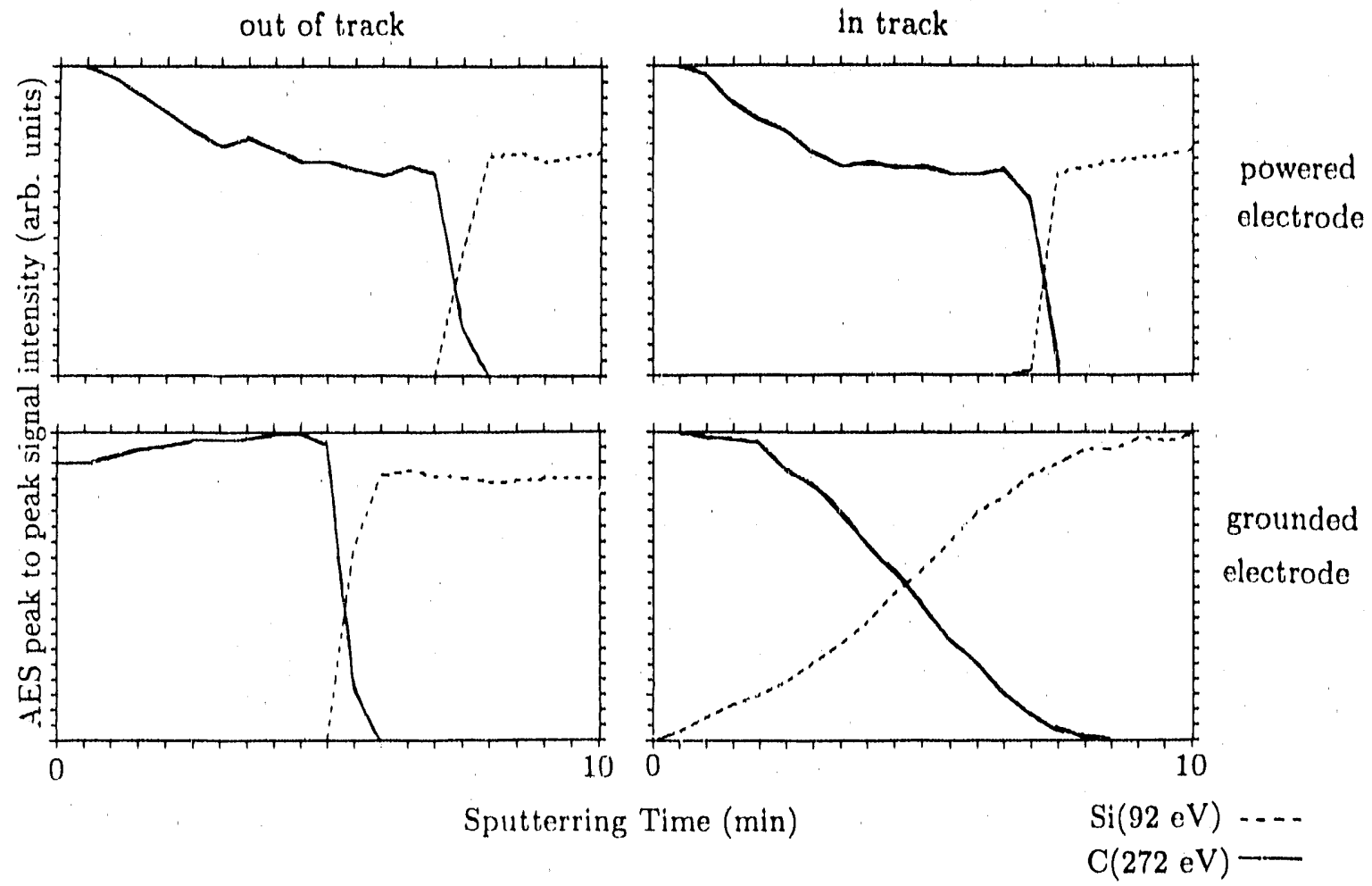

$\mathrm{XBi}, 896-2530$

Figure 3.4: Peak to peak auger intensities for carbon and silicon as a function of argon ion sputter time for areas in the scratch tracks and away from the tracks of the areas shown in the previous figure. A large amount of intermixing is evident in the scratch track on the soft film formed on the grounded electrode. 
scratch. The film formed on the powered electrode was not measurably damaged at this load level, demonstrating its hardness and strong adhesion. The interface appears equally sharp both in the scratch track and away from the track. The film formed on the grounded electrode, however, shows a large amount of damage and a strong intermixing of carbon and silicon in the scratch track, demonstrating the poor mechanical properties of this film.

A markedly high compressive stress of 1.5-3.0 GPa was measured for films formed on the powereci electrode ( $\Gamma$ ig. 3.2). In order for the films to withstand such a high stress and remain attached to the silicon substrate, there must be good adhesion, probably due to strong $\mathrm{Si}-\mathrm{C}$ chemical bonds which are expected to be present at the interface. Films formed at the grounded electrode did not exhibit a measurable stress.

Density is also significantly different between the films produced on the two types of electrodes. The value for the carbon film produced on the powered electrode; 1.7 $\mathrm{g} / \mathrm{cm}^{3}$, is in close agreement with those previously reported[7]. Although this value is much lower than that of diamond and closer to that of graphite (3.52 and 2.27 $\mathrm{g} / \mathrm{cm}^{3}$, respectively), this does not imply that the film has a sparse three dimensional structure. In view of the considerable hydrogen content expected in these films, the number density (number of atoms per unit volume) could be close to that of diamond, and hard a-C:H films must essentially consist of a dense hydrocarbon phase[8]. Films formed at the grounded electrode were less dense $\left(1.3 \mathrm{~g} / \mathrm{cm}^{3}\right)$ than powered electrode films.

\subsection{FTIR Spectra and Optical Gap}

Fourier transform infrared spectroscopy of a- $\mathrm{C}: \mathrm{H}$ films was carried out in order to study the hydrogen content and bonding within the films. Fig. 3.5 compares the FTIR spectra of carbon films formed on the powered and on the grounded electrodes, in two frequency regions associated with $\mathrm{C}-\mathrm{H}$ stretching and $\mathrm{C}-\mathrm{H}$ bending vibrations. The soft carbon film formed at the grounded electrode shows absorption bands characteristic of methyl groups $\left(1375,2878\right.$, and $\left.2958 \mathrm{~cm}^{-1}\right)$ as well as methylene groups $\left(1460,2930 \mathrm{~cm}^{-1}\right)[9]$. The frequencies of the above bands are close to those reported for alkanes [9], implying that the carbon films formed on the grounded electrode are 


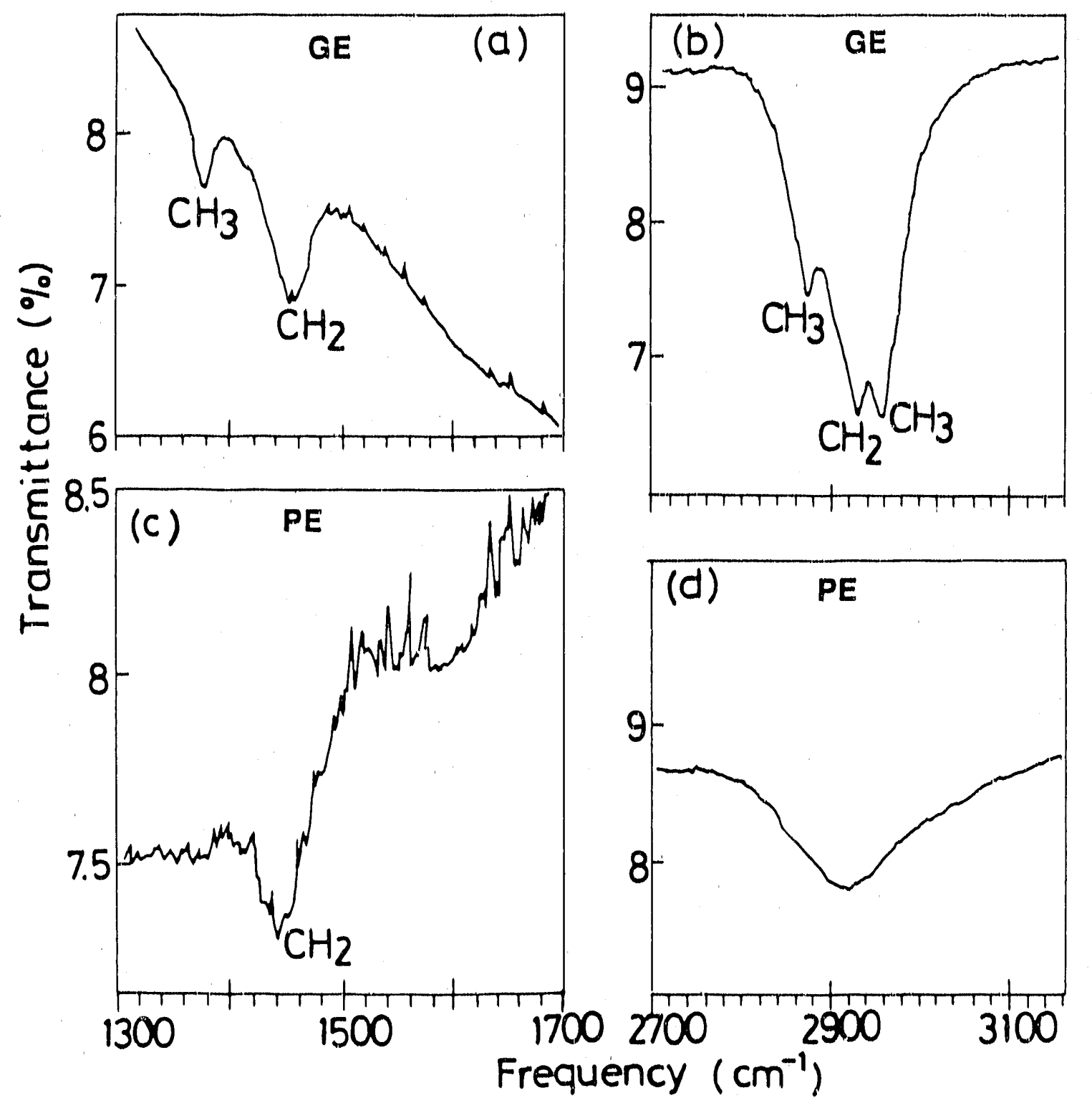

Figure 3.5: FTIR spectra of a- $\mathrm{C}: \mathrm{H}$ films formed on the grounded[(a) and (c)] and powered [(b) and (d)] electrodes. Film thicknesses for the grounded and powered electrode films were 1.27 and $2.15 \mu \mathrm{m}$, respectively. The spectrum for the film formed on the grounded electrode shows absorptions due to $\mathrm{CH}_{3}$ which are not apparent in the powered electrode film spectrum. 
made up of a structure which is much less saturated than even the polyacetylene materials. It is also likely that they are composed of grains rich in methyl group chain branches, due to the large intensity of the methyl group bands.

In comparison, the carbon films formed on the powered electrode show no such distinct $\mathrm{CH}_{3}$ bands, indicating more extensive carbon - carbon linkages, or more $\pi$ bonding, or both. The broad $\mathrm{C}-\mathrm{H}$ stretching band at around $2920 \mathrm{~cm}^{-1}$ is similar to that reported by Dischler et al[10] for an a-C:H fllm produced from benzene by PACVD. In that study, the band was deconvoluted into four overlapping bands attributed to $\mathrm{sp}^{2} \mathrm{CH}$ and $\mathrm{sp}^{3} \mathrm{CH}_{2}$ stretching vibrations. The band assigned to the $\mathrm{C}-\mathrm{H}$ bending of $\mathrm{CH}_{2}$ is centered at $1445 \mathrm{~cm}^{-1}$, red shifted with respect to the same band for the grounded electrode, at $1460 \mathrm{~cm}^{-1}$. This red shift is suggestive of cyclization of the carbon skeleton[10]. Cyclization will promote extension of the three dimensional network and could cause stress inside the films. Moreover, the appearance of a weak band near $1600 \mathrm{~cm}^{-1}$ could be associated with graphite-like or conjugated $\mathrm{sp}^{2}$ clusters, which must also be responsible for the strong absorption of visible light by the carbon films produced on the powered electrode.

The IR spectra also suggest a large difference in hydrogen content between the two films. Based on the molar extinction coefficients for the methylene and methyl bending vibrations (14.9 and $15.0 \mathrm{l} / \mathrm{mole}-\mathrm{cm}$ according to the work of Tibbitt et al[11]), the concentration of methylene and methyl groups in the carbon produced on the grounded electrode are estimated to be 20 and 12 mmole per gram of film (mmole/ $\mathrm{g}_{f}$ ), respectively. Thus, in the grounder electrode deposit more than one in three of the carbon atoms is either bonded as $-\mathrm{CH}_{2-}$, or $-\mathrm{CH}_{3}$. For the carbon film formed on the powered electrode, on the other hand, the methylene concentration is estimated to be about $5 \mathrm{mmole} / \mathrm{g}_{f}$, lower by a factor of four compared to that in the grounded electrode carbon, while the methyl concentration is below the limit of detection. The lack of information for tertiary $\mathrm{C}$ - $\mathrm{H}$ groups does not allow evaluation of total hydrogen content, but, from the values given, a much lower hydrogen content is evident for the carbon films formed on the powered electrode.

An optical gap of $3.1 \mathrm{eV}$ was determined from the Tauc plot for a film formed on glass at the grounded electrode. The dark, hard films formed at the powered electrode 
exhibited a much smaller optical gap of $1.2 \mathrm{eV}$. The lower optical gap present in films formed at the powered electrode is likely a result of an increased concentration of $\mathrm{sp}^{2}$ clusters in this material[6].

Raman and SIMS investigations of films formed on the powered and grounded electrodes were also carried out. While the aforementioned techniques demonstrate how the films differ significantly in both their mechanical and chemical properties, Raman spectroscopy and SIMS failed to differentiate between the coatings. Typical Raman and SIMS spectra ior the a-C:H films are shown in Fig. 3.6. and Fig. 3.7, respectively. The Raman spectra for all the films was typical of those previously presented in the literature for amorphous carbon films[13]. No systematic variations in the Raman spectra were observed as a function of deposition conditions. SIMS spectra also did not exhibit any systematic variations with deposition conditions. Although SIMS is sensitive to hydrogen, the signal due to the background pressure of hydrogen in the chamber, apparently obscures any variations in $\mathrm{H}^{+}$intensity between films. It is also possible that the ion sputtering, used to generate secondary ions in SIMS, alters the structure and composition of the $\mathrm{a}-\mathrm{C}: \mathrm{H}$ films in such a way as to make their secondary ion mass spectra indistinguishable.

\subsection{Discussion}

The results in this chapter demonstrate the significant differences in properties between films formed on the powered electrode and those formed on the grounded electrode. The mechanical properties of films formed on the powered electrode are notably superior. The FTIR spectra indicated that the powered electrode films contain less hydrogen. Powered electrode films have a relatively small methyl group concentration. Conversely, the large methyl group concentration in the grounded electrode films indicates that a lack of crosslinking exists in these soft films. The large optical gap of the soft films is typical of a highly hydrogenated polymeric material. The decreased optical gap of the powered electrode films is thought to result from the presence of conjugated clusters of graphitelike or $\mathrm{sp}^{2}$ carbon. These graphitelike clusters are likely responsible for a feature in the FTIR spectrum, a.t $1600 \mathrm{~cm}^{-1}$, which appears only for the powered electrode films. 


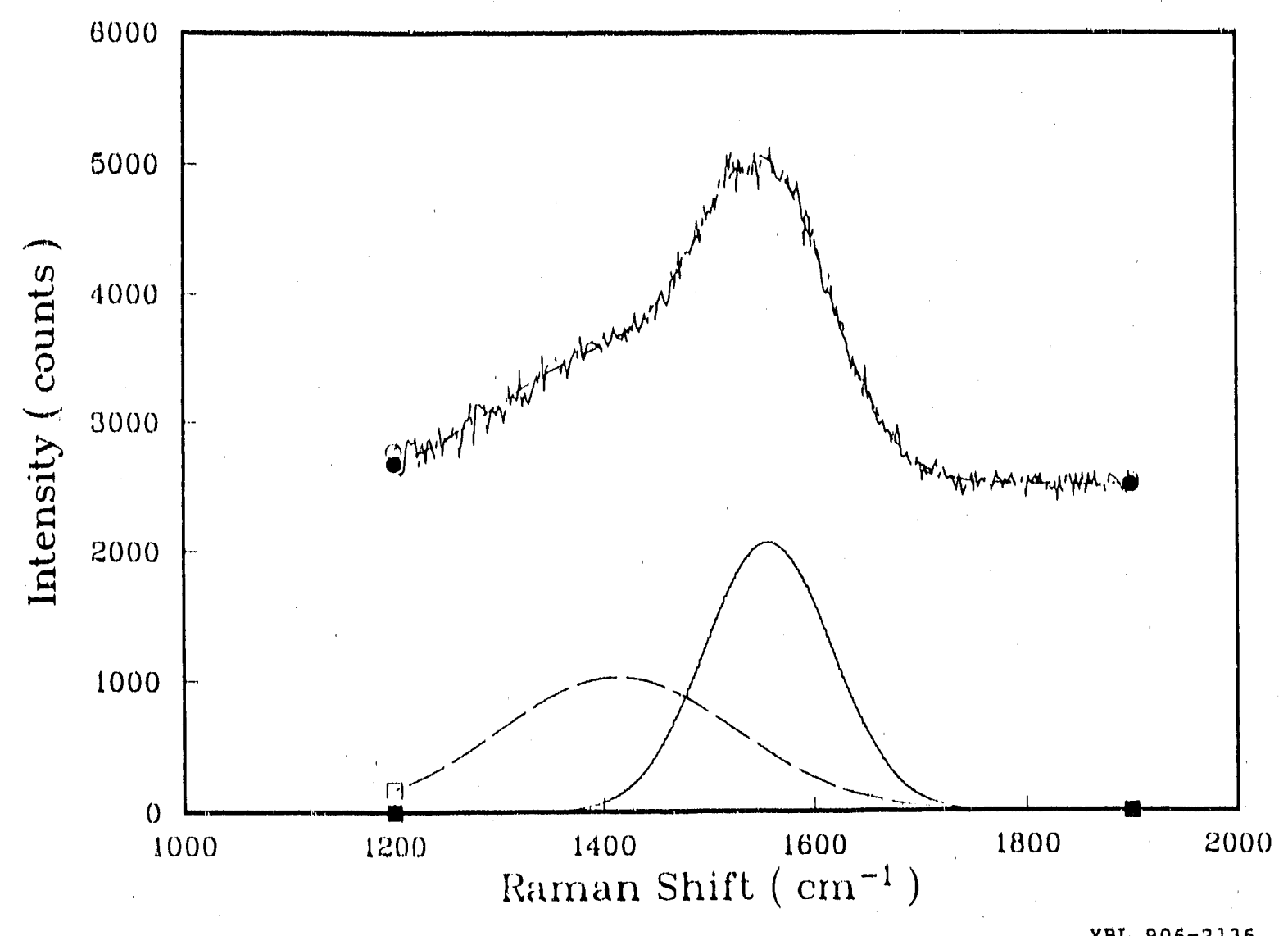

Figure 3.6: Typical Raman spectrum for an a-C:H film. 

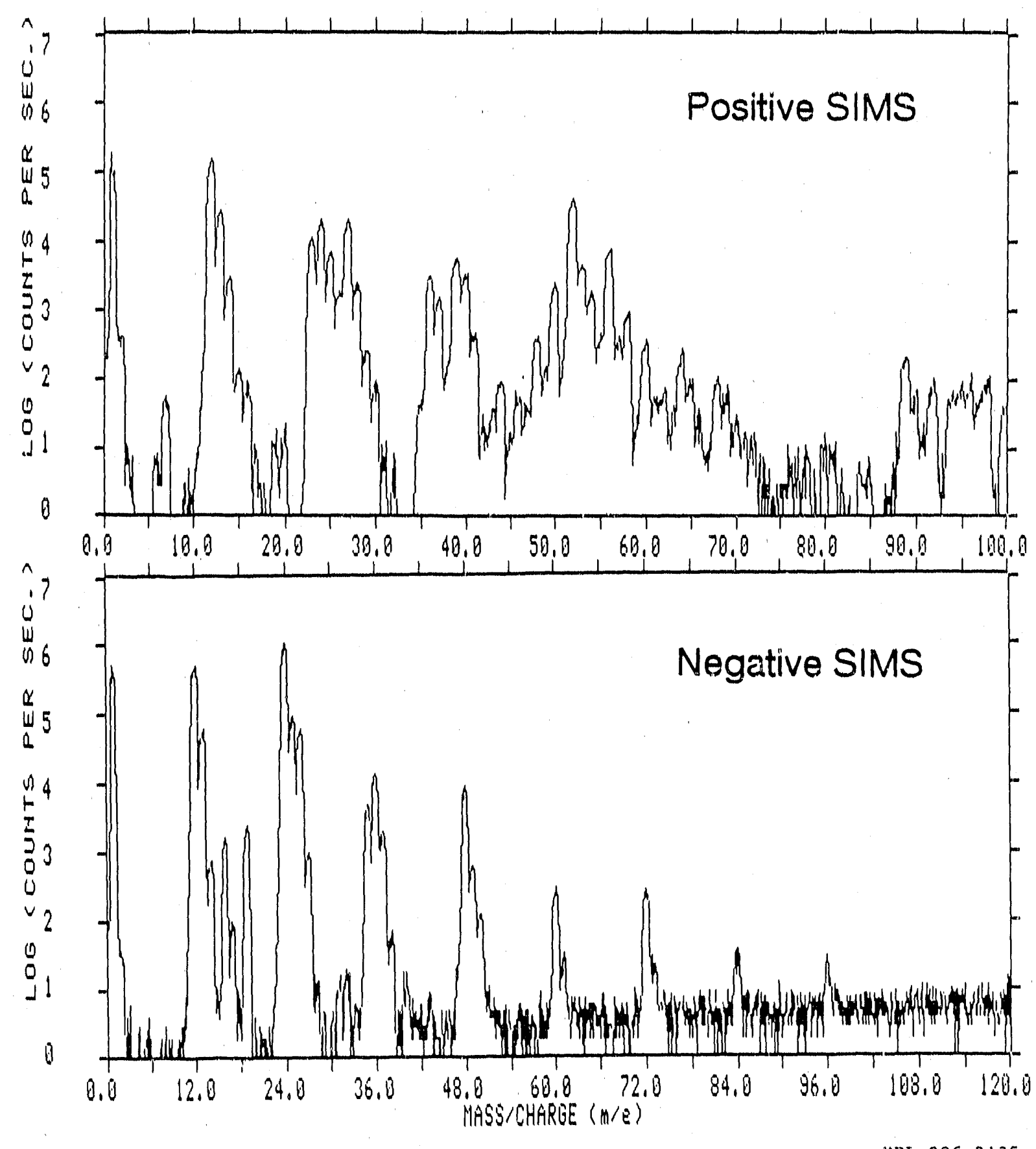

Figure 3.7: Typical SIMS spectra for an a-C:H film. 
While the large differences in the properties of the films are clear, the origin of these differences is not. The most obvious difference in the growth conditions between the two electrodes is the large self-bias voltage present at the powered electrode. Positive ions created in the plasma which diffuse into either of the electrode sheaths are accelerated towards the sample surfaces at their respective electrodes. However, ions will strike the surface of powered electrode samples with a much greater energy than they will strike the surface of grounded electrode samples. This ion energy difference is the most convenient and perhaps the most likely explanation for the noted differences in mechanical properties. However, the plasma, species actually responsible for the deposition have not been identified. They may be plasma ions or they may be free radicals or both. The flux of reactive ions and radicals is also not known. To understand the origin of the differences in film properties, the growth mechanism of a-C:H films must be better understood. Therefore, more knowledge must be gained regarding the plasma species present and their respective roles in the deposition process. 


\section{Bibliography}

[1] Y. Catherine and P. Couderc, Thin Solid Filins 144, 265 (1986).

[2] P. Couderc and Y. Catherine, Thin Solid Films 146, 93 (1987).

[3] K. Yamamoto, Y. Ichikawa, T. Nakayama, and Y. Tawada, Jap. J. Appl. Phys. 27, 1415 (1988).

[4] J.W. Zou, K. Reichelt, K. Schmidt, and B. Dischler, J. Appl. Phys. 65, 3914 (1989).

[5] B. Meyerson and F.W. Smith, J. Non-Cryst. Solids 35/36, 435 (1980).

[6] E. Staryga, A. Lipinski, S. Mitura, and Z. Has, Thin Solid Films 145, 17 (1986).

[7] J. Robertson, Adv. Phys. 35, 317 (1986).

[8] J.C. Angus, Thin Solid Films 142, 145 (1986).

[9] R.M. Silverstein, C.T.C. Bassler, and T.C. Morrill, Spectrometric Identification of Organic Compounds, 4th ed. (Wiley, New York, 1981).

[10] B. Dischler, A. Bubenzer, and P. Koidl, Solid State Commun. 48, 105 (1983).

[11] J.M. Tibbitt, M. Shen, and A.T. Bell, J. Macromol. Sci. Chem. A 10, 1623 (1984).

[12] M.A. Tamor, J.A. Haire, C.H. Wu, and K.C. Haas. Apply. Phys Lett. 54, 123 (1989).

[13] M. Ramsteiner, J. Wagner, Ch. Wild, and P. Koidl, J. Appl. Phys. 62, 729 (1987). 


\section{Chapter 4}

\section{Plasma Characterization}

\subsection{Introduction}

Characterization of the species impinging on the sample surface is necessary if the growth mechanism of a-C:H films is to be understood. This chapter explains the results obtained by mass analysis of the ionic and neutral species extracted through an orifice located in the electrode where the sample would otherwise be. Mass analysis was performed at both the powered electrode and at the grounded electrode. The ionic species impinging on the sample surfaces at both electrodes were identified. The relative total ion flux at the electrodes was measured and an upper limit on the absolute ion flux was determined. The impinging neutral species were also identified. The flux of methyl radicals onto the sample surface was determined, and was found to be sufficiently high so as to be able to account for the a-C:H deposition rate.

\subsection{Neutrals}

In order to understand the contribution of radical species to a-C:H film growth, mass spectra of the neutral species incident on the powered and grounded electrodes were collected, as shown in Fig. 4.1. The signal in the 12 to 15 amu range contains minor contributions from plasma radicals, as will be deduced later. The methane peaks decreased to about half of that observed in mass spectra taken without the ignition of the plasma discharge. In place of this intensity, a strong hydrogen peak and weak features due to $\mathrm{C}_{2} \mathrm{H}_{x}$ species emerged as a result of plasma reactions.

In order to determine the signal intensity due to plasma radical species, all inten- 


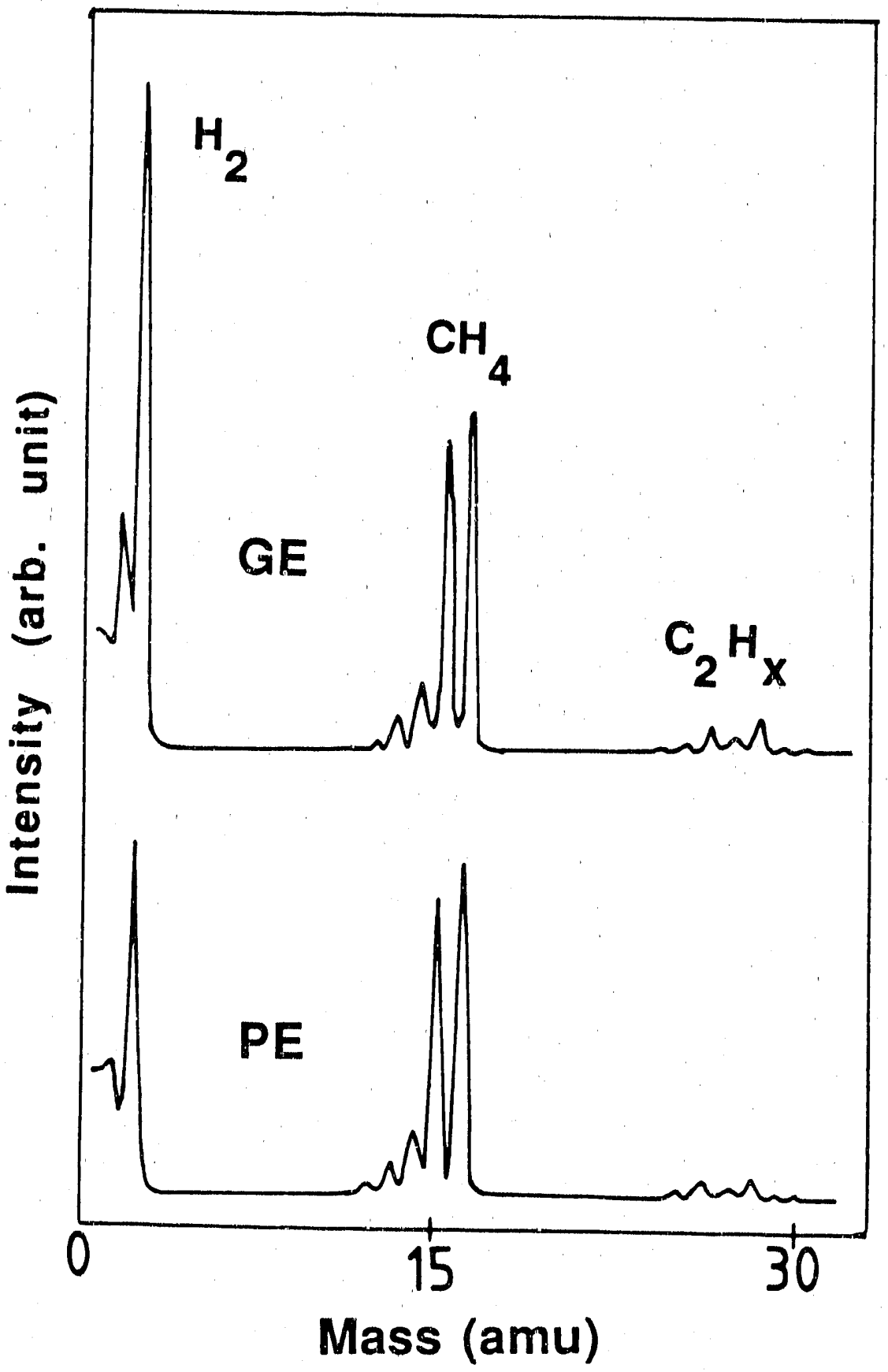

Figure 4.1: Mass spectra of neutrals incident on the grounded electrode (GE) and the powered electrode (PE). 
sity in the measured mass spectra, originating from the nonradical component of the plasma species, must be subtracted. This includes the parent peak for each nonradical component, as well as the intensity generated in various other mass channels, as a result of electron impact dissociation reactions occurring in the ionizer region of the mass spectrometer. Cracking patterns spectra and sensitivities of the mass analyzer for all the relevant hydrocarbon species were measured in this system in advance, by leaking through the pure gases to measured pressures in the plasma chamber. According to these calibrations, the weak features from $\mathrm{C}_{2}$ species can be deconvoluted into the contributions from ethane, ethylene, and acetylene as shown in Fig. 4.2, which is calculated for the spectrum obtained through the grounded electrode. A deconvolution of the features for the powered electrode resulted in a similar ratio. The main production channels of these stable $\mathrm{C}_{2}$ species in a methane plasma have been discussed by Tachibana et al[1].

The sum of the partial pressures of the measured plasma species must coincide with the measured pressure in the system. Once the intensities resulting from the specific species are known, their respective partial pressures can be obtained from the analyser sensitivities as measured for the pure compounds. The result is shown in Fig. 4.3. This figure represents the partial pressures within a meari free path of the sampling orifice in the lower electrode. As can be seen in Fig. 4.3, there is a measurable difference between the powered and grounded electrodes in terms of the individual partial pressures. However, summation of the partial pressures evaluated in this way results in an almost identical total pressure for both electrodes (ca. $65 \mathrm{mTorr}$ ), which is only about 3 mTorr below that measured with a capacitance manometer at a point away from the plasma region. This indicates a homogeneous total gas pressure within the plasma chamber, and provides an independent assessment of the mass analyzer system calibration.

Of great interest is the contribution made by the radicals, to the neutral signal intensity. This contribution, neglected in Fig. 4.3, can partly account for the slightly higher total pressure measured with the pressure gauge, as compared to the calculated one. The mass signals due to radical species in the 12 to 15 amu range can be deduced by careful subtraction of nonradical components, namely the signal intensities due 


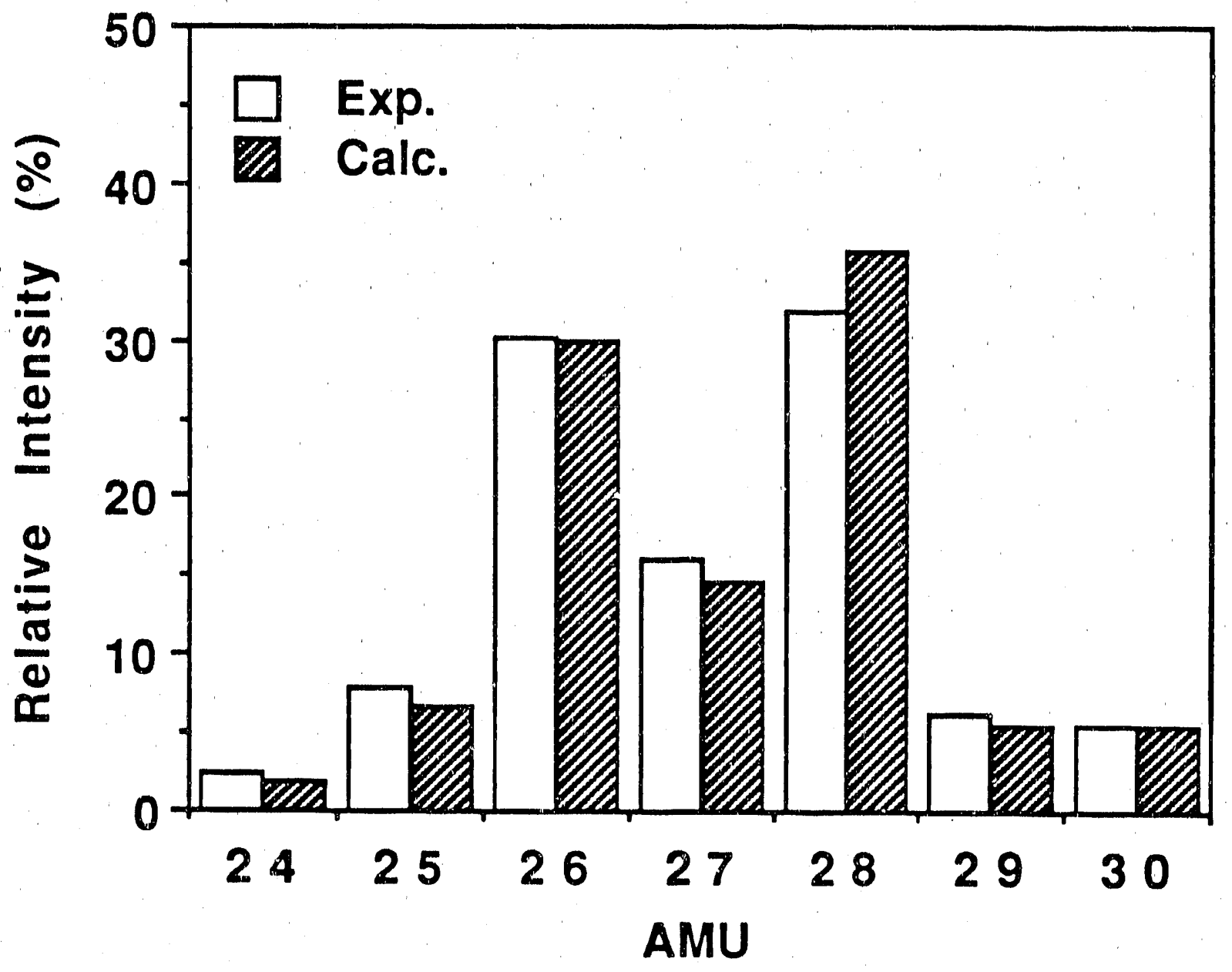

XBL $896-2524$

Figure 4.2: Experimental and calculated intensities of the mass spectrometric intensities in the cracking of various $\mathrm{C}_{2} \mathrm{H}_{x}$ species. Intensities are calculated based on the optirnum concentration ratios (calculated as $\left[\mathrm{C}_{2} \mathrm{H}_{6}\right]:\left[\mathrm{C}_{2} \mathrm{H}_{4}\right]:\left[\mathrm{C}_{2} \mathrm{H}_{2}\right]=0.45$ : $0.27: 0.28$ ) and the cracking pattern spectra of the pure gases taken with the same apparatus. 


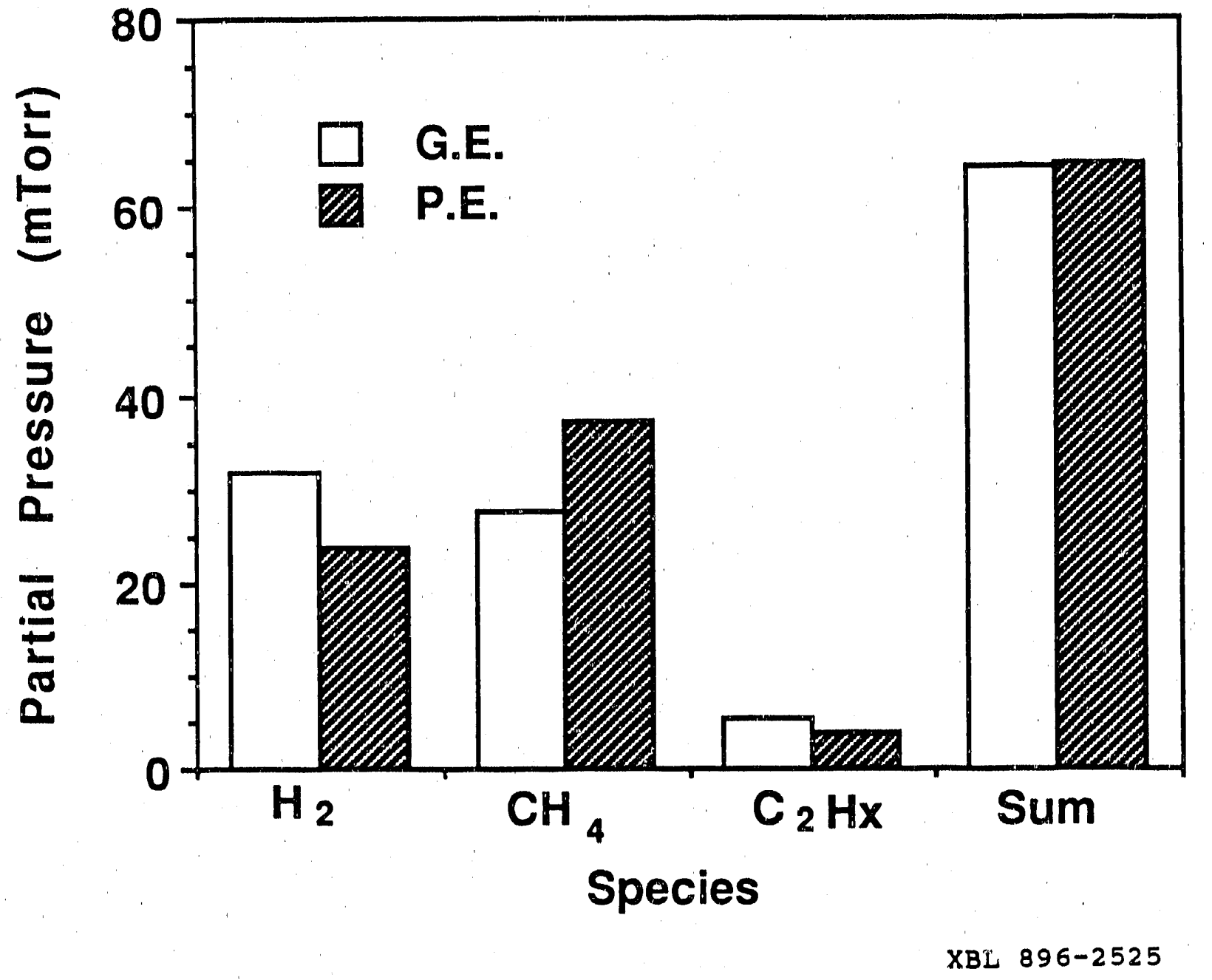

Figure 4.3: Partial pressures of neutral species above the substrate at the GE and the PE. 
to the fragments of methane and $\mathrm{C}_{2}$ species, produced in the mass spectrometer ionization chamber. The result is shown in Fig. 4.4. The only signal observed at a level significantly greater than the experimental variation is that of $15 \mathrm{amu}$, showing that the methyl radical is dominarit. This is in agreement with the predictions of Tachibana et al[1] and Kline et al[2]. Fig. 4.4 also indicates that there is no significant difference in radical flux between the powered and grounded electrodes. From these data we can conclude that the maximum possible contribution to film growth of radicals produced in the plasma is not significantly different between the powered and grounded electrodes.

In order for the result shown in Fig. 4.4 to be used for an estimation of the methyl radical density in our methane plasma, some information about the sticking coefficient of methyl radicals is required. The signal intensity measured with the mass analyzer depends on a steady state concentration of the relevant species in the high vacuum chamber for the mass analysis. This concentration is not solely determined by the flux of the species coming in through the orifice, but depends as well on the sticking probability onto the chamber walls. The pressure inside the chamber is maintained at $10^{-8}$ Torr, making collisions with walls the predominant scattering mechanism.

By blocking the direct pathway between the lower orifice and the mass spectrometer ionization chamber, the signal intensity of any species with a high sticking probability would be significantly attenuated. Fig. 4.5 compares the relative radical intensities with and without such a shield. Only a slight decrease in the $\mathrm{CH}_{3}$ signal intensity is observed when such a shield is in place. The difference is almost within the experimental variation. This indicates a fairly small sticking coefficient for the methyl radicals onto the stainless steel chamber walls, which in turn allows an estimation of methyl radical density in the plasma. The ionization cross section of the methyl radical is the only other important parameter to be taken into account. This will be explained in more detail in the discussion section, in relation to the deposition rate.

\subsection{Ions}

The mass spectra of positive ions incident on the powered and grounded electrodes 


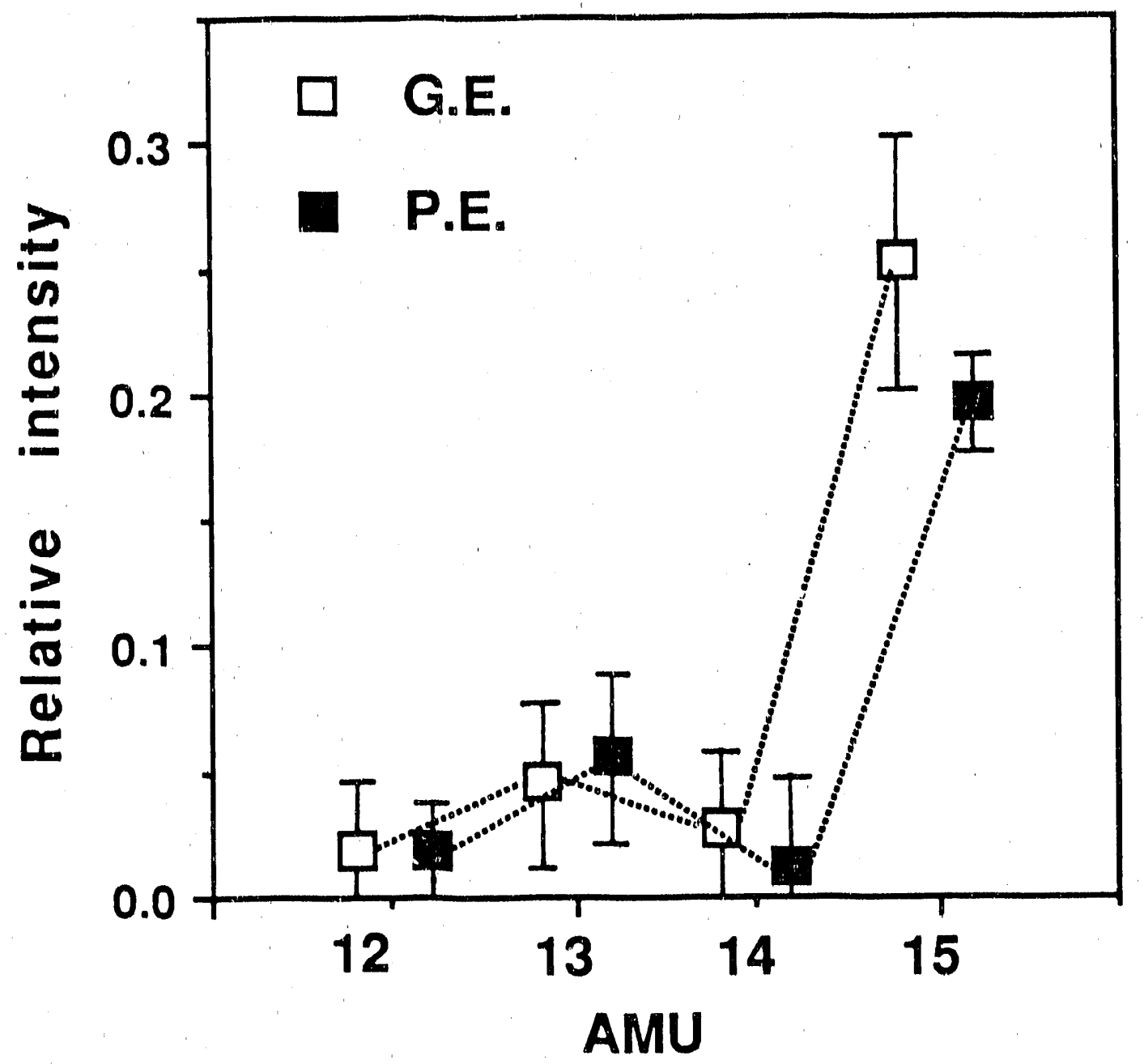

XBL $896-2526$

Figure 4.4: Relative intensities of the mass $12-15$ signals due to neutral radical species, for the $G E$ and the $\mathrm{PE}$. The $\mathrm{CH}_{3}$ radical is the predominant radical species in the plasma. 


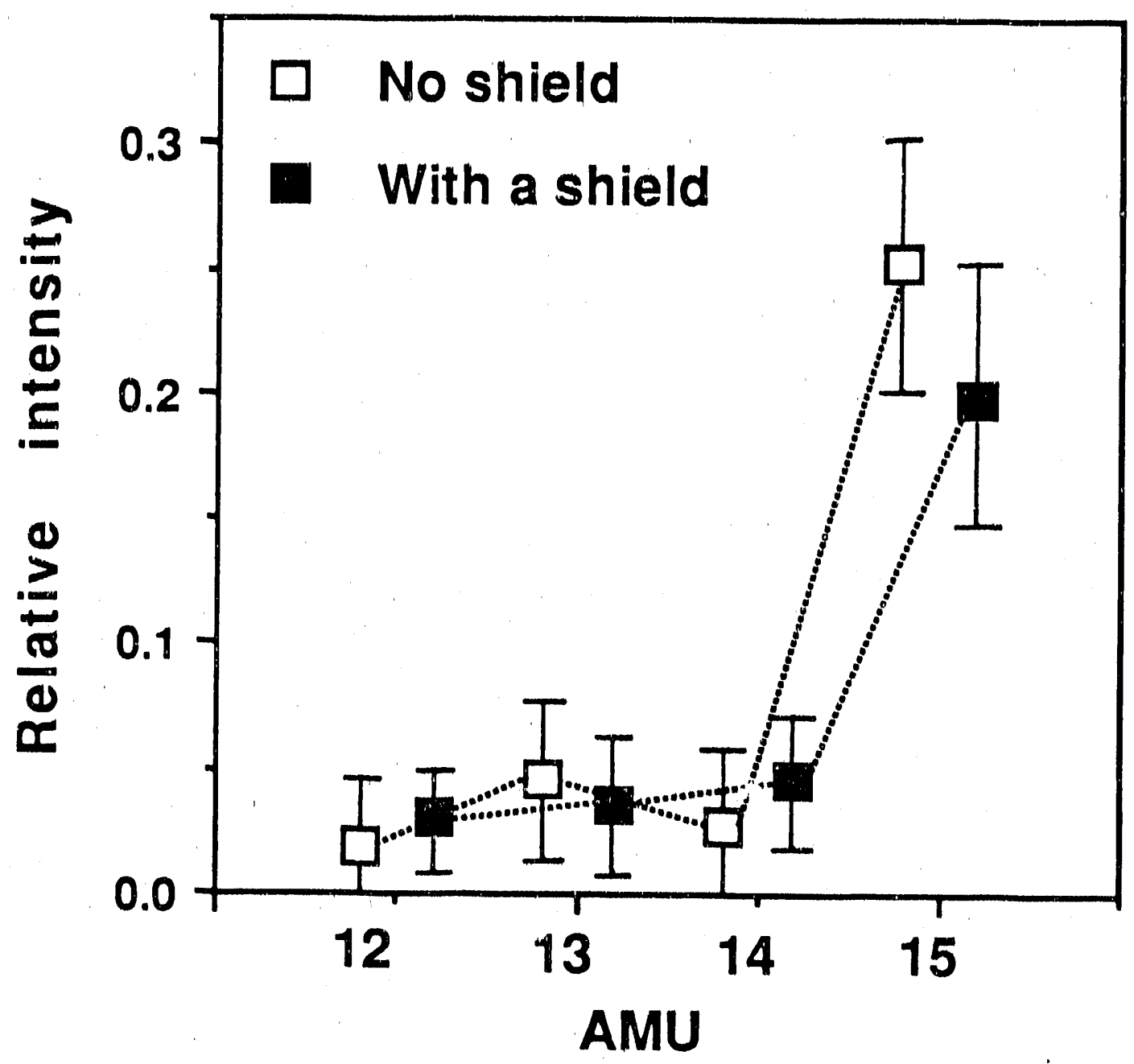

XBL $896-2527$

Figure 4.5: Effect of direct path shielding on the detested neutral radical intensities. Intensities with and without a shield are shown. The lack of a measurable change in the 15 amu signal intensity indicates a small sticking coefficient for the methyl radicals with the chamber walls. 
were measured by turning off the ionizer in the mass spectrometer. The spectra are shown in Fig. 4.6. In addition to the the primary ions $\mathrm{CH}_{3}{ }^{+}$and $\mathrm{CH}_{4}{ }^{+}$which are species produced by electron impact lonization of methane, secondary ions are also observed in relatively high ratios at the grounded electrode. These secondary ions, like $\mathrm{CH}_{5}{ }^{+}$and $\mathrm{C}_{2} \mathrm{H}_{5}{ }^{+}$, are produced through ion molecule reactions. On the other hand, at the powered electrode, $\mathrm{CH}_{3}^{+}$is the most dominant and $\mathrm{CH}_{2}{ }^{+}$and $\mathrm{CH}^{+}$ions can also be seen, together with minor peaks due to secondary ions as mentioned above.

The large difference in spectral features, as seen in Fig, 4.6 can be explained in terms of the larger potential drop near the powered electrode, as compared to the grounded electrode. Because of the large potential drop at the powered electrode, secondary electrons emitted there are accelerated into the plasma at much greater energies than those emitted at the grounded electrode. As a result, electron impact ionization of methane occurs at a much higher electron energy near the powered electrode. This is more likely to produce less-hydrogenated fragment ions. Also, ions produced throngh electron impact near the powered electrode are much more rapidly accelerated towards the electrode by the large potential drop than are ions produced near the grounded electrode. The probability that these ions react with methane molecules is thus decreased and the relative flux of the secondary ions onto the powered electrode is reduced.

By measuring and differentiating the ion signal intensity as a function of retarding voltage, the ion energy distributions with respect to the ground potential of the mass spectrometer were obtained. Fig. 4.7 shows the ion energy distributions measured in this way for $\mathrm{CH}_{2}^{+}, \mathrm{H}^{+}$, as well as for the total ions. In the case of the grounded electrode, the energy peaks are found, irrespective of the species, in the 15 to 20 $\mathrm{eV}$ range. This corresponds to the plasma potential in the glow space as shown in Fig. 2.4.

Since the mass spectrometer and all other chamber elements located below the orifice in the lower electrode are at ground potential, ions incident on the lower electrode with energies from 350 to $430 \mathrm{eV}$ decelerate to energies from 0 to $80 \mathrm{eV}$ before being detected. Therefore, the energies with which ions strike the powered electrode 


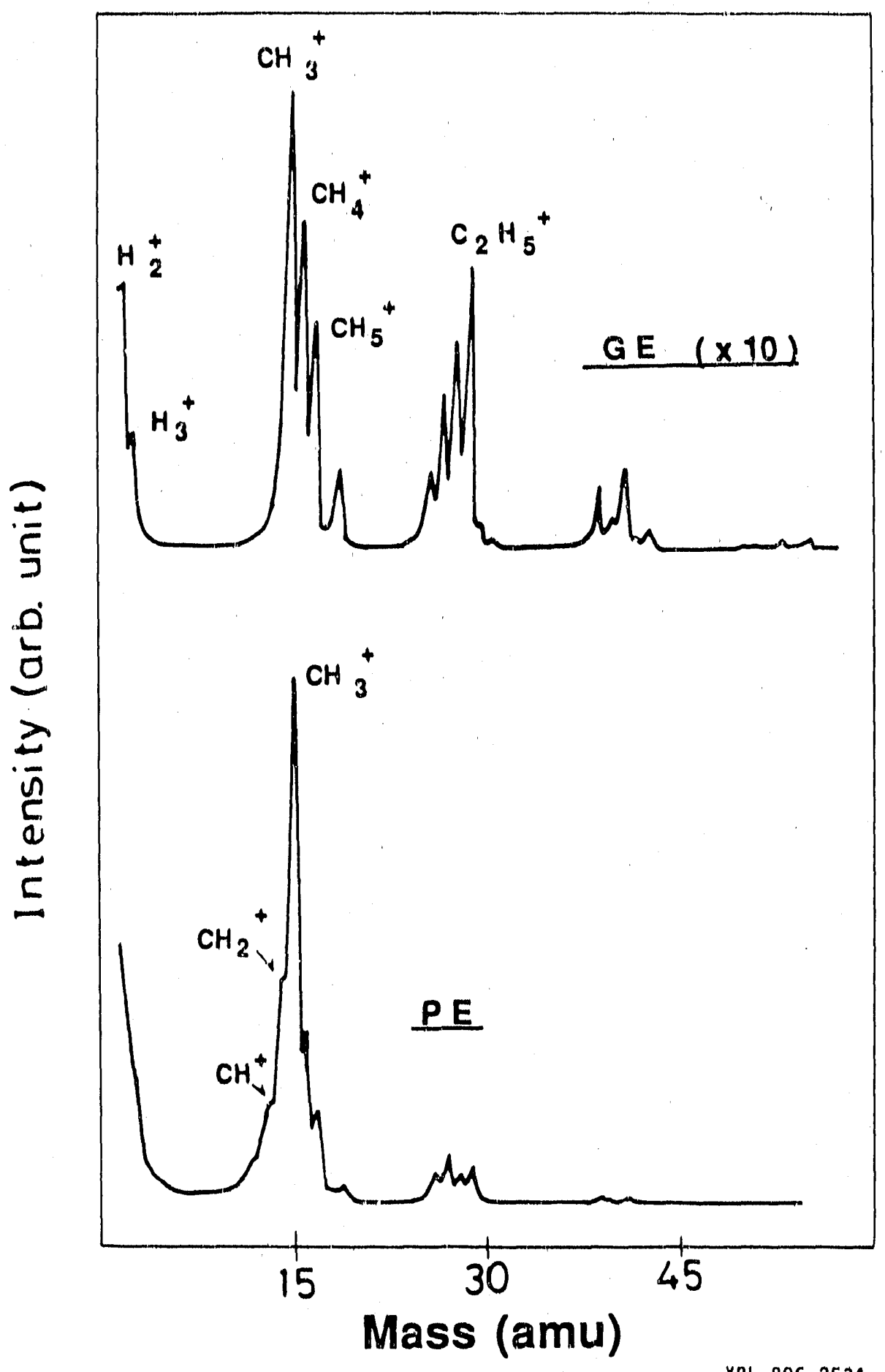

XBL $896-2534$

Figure 4.6: Mass spectra of ions incident on the GE and the PE. The intensity for the grounded electrode spectrum has been magnified by a factor of 10 . 


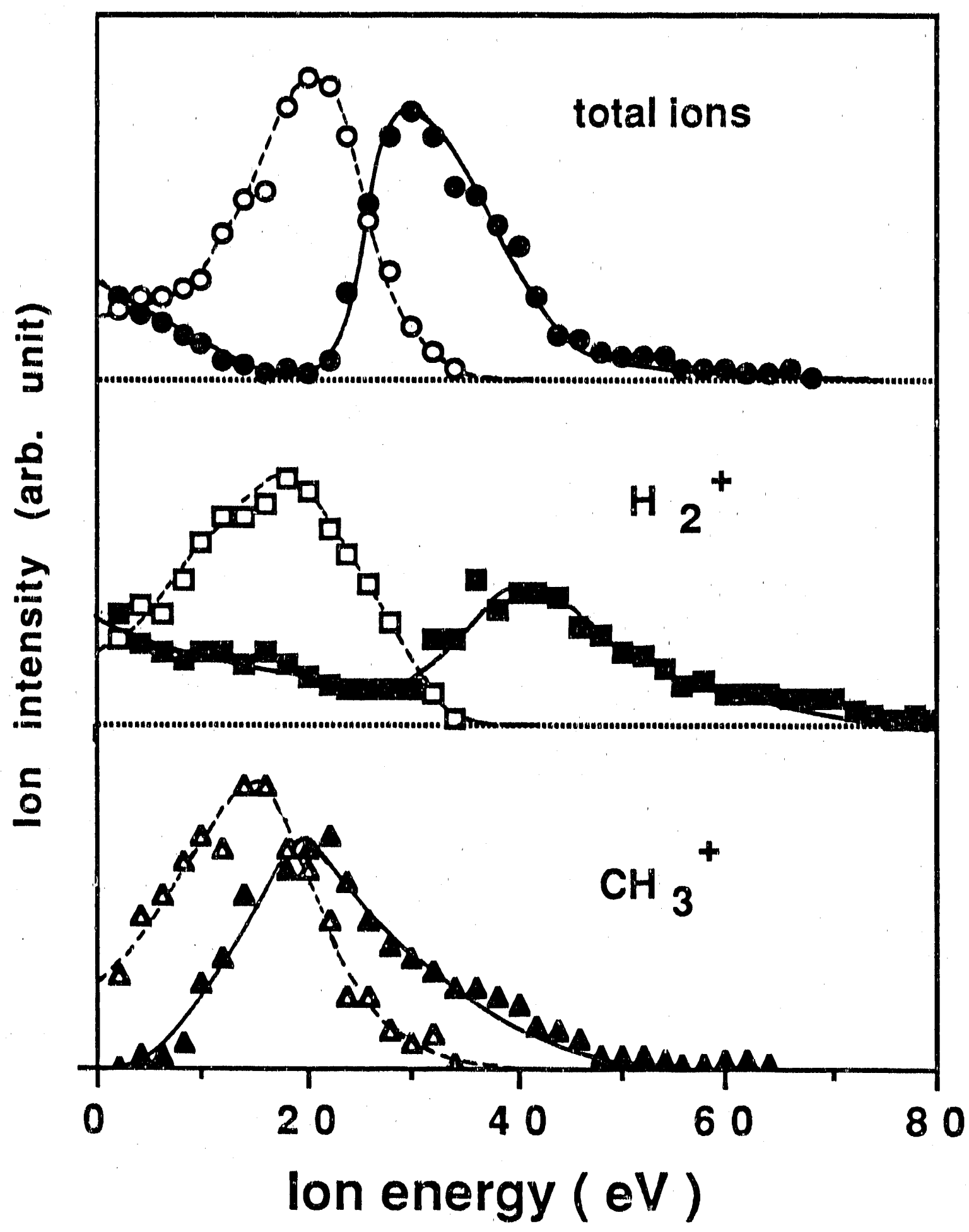

Figure 4.7: Ion signal intensity vs ion energy for the total ions, $\mathrm{H}_{2}^{+}$, and $\mathrm{CH}_{3}^{+}$ions incident on the grounded electrode (open shapes) and the powered electrode (solid shapes). The energies with which the ions strike the powered electrode are $350 \mathrm{eV}$ greater than those measured at the mass spectrometer and listed here. 
are $350 \mathrm{eV}$ higher than the energies shown in Fig. 4.7. The ion energy distributions for the powered electrode are also more complex than that for the grounded electrode. The distribution for hydrogen ions, for example, shows a high energy peak together with a secondary peak on the low energy side. This is likely to be related to an if modulation of ion energy, which takes place when the time required for an ion to traverse the cathode sheath is comparable to or shorter than the rf cycle. In fact, a much heavier ion, $\mathrm{CH}_{3}{ }^{+}$, shows only a single energy peak close to the plasma potential. This last point implies that ions which have entered the sheath edge impinge on the powered electrode with a kinetic energy almost equivalent to the potential difference between the electrode and the plasma bulk, without any significant loss of energy due to collision with gas phase species. Thus, ions impinging on the grounded electrode have less than $20 \mathrm{eV}$ of kinetic energy on average, but ions incident on the powered electrode exceed $350 \mathrm{eV}$ in kinetic energy,

The total ion current density was measured at both the powered and grounded electrodes using the Faraday cup arrangement described in the experimental section. With the Faraday cup biased at $-400 \mathrm{~V}$, the current density at the powered electrode was measured as $1.7 \times 10^{15} \mathrm{~cm}^{-2} \mathrm{~s}$. The current density measured in this way at the grounded electrode was $0.4 \times 10^{15} \mathrm{~cm}^{-2} \mathrm{~s}$. These current density measurements are upper limits to the real ion current density measurements, as explained in the experimental section. The relative current density measurements should be accurate however, as current doubling effects should occur at the same magnitude for both electrode conditions. It is thus concluded that, under the standard deposition conditions, the positive ion current flux is about 4 times greater at the powered electrode as compared to the grounded electrode.

\subsection{Discussion}

\section{Radicals}

The most dominant radical is the methyl radical (Fig. 4.4), which possesses a rather small sticking coefficient, as noted by the lack of an intensity decrease with a shield in place (Fig. 4.5). The dominance of methyl radicals is expected from the individual cross section data provided by Melton and Rudolph[3], which favors the 
formation of methyl radicals over other fragments by electron impact dissociation of methane. The high concentration of methyl radicals is also consistent with the low reactivity of methyl radicals with methane which results in a long methyl radical lifetime[4]. If the main role of the methyl radical is to provide the bulk mass of the films by polymerization, the methyl radical density must be high enough to account for the observed mass depositio? rate. In order to evaluate this methyl radical density based on the result shown in Fig. 4.4, the ionization cross section of methyl radical in our mass analyzer must first be estimated. To the author's best knowledge, the only available cross seciion data for methyl radical related species are for the deuterated species, $\mathrm{CD}_{3}$, reported by Baiocchi et al[5]. For an electron energy of $75 \mathrm{eV}$, like that used in our mass spectrometer system, the total ionization cross section of $\mathrm{CD}_{3}$ was measured at $2.8 \times 10^{-16} \mathrm{~cm}^{2}$. In this work, an equal cross section was assumed for $\mathrm{CH}_{3}$. On the other hand, the total ionization cross section of methane, which was used as a reference species for the present estimation, varies significantly as determined hy different workers. In this work, the value of $3.8 \times 10^{-16} \mathrm{~cm}^{2}$, as reported by Melton et al[3] was used. Using the same intensity units as used in Fig. 4.4, $1 \mathrm{mTorr}$ of methane in the plasma chamber produces an integrated intensity over the total ionization fragments of about 2.0, while according to Fig. 4.4, the signal intensity due to methyl radical is approximately 0.2 .

Due to the small sticking probability of the methyl radical with the chamber wall, its average residence time in the high vacuum chamber where mass analysis occurs, is expected to be similar to that of the stable methane molecule. Therefore the numerical values given above are sufficient for estimating the methyl radical densit:" in the methane plasma. The ratio of ionization cross sections multiplied by the signal intensity ratio, gives the dinısty ratio of methyl radicals to methane molecules. The methane pressure and thus density is known, so the radical density is easily calculated. From the signal inter ity ratios and the ionization cross sections used, the partial pressure of methyl radical should be about $0.14 \mathrm{~m}$ Torr, which is equ to a radical density of $4.5 \times 10^{12} \mathrm{~cm}^{-3}$ at $300 \mathrm{~K}$. For this density, the radical flux to the substrate is calculated to be about $7 \times 10^{16} \mathrm{~cm}^{-2} \mathrm{~s}^{-1}$.

The mass deposition rate, as measured on the powered electrode, for example, 
was $4.1 \times 10^{-8} \mathrm{~g} \mathrm{~cm}^{-2} \mathrm{~s}^{-1}$. By assuming an atomic ratio of hydrogen to carbon in the film to be $1: 1$, the above growth rate requires deposition of $C_{1}$ species at the rate of $1.9 \times 10^{15} \mathrm{~cm}^{-2} \mathrm{~s}^{-1}$. Due to the much lower atomic mass of hydrogen as compared to carbon, this value is not very sensitive to the assumed value of the atomic atio of hydrogen to carbon. A similar calculation for the grounded electrode leads to a smaller rate of $1.0 \times 10^{15} \mathrm{~cm}^{-2} \mathrm{~s}^{-1}$.

By comparing the estimated radical flux with these growth rates, it can be seen that methyl radicals alone could adequately account for the deposited mass. If methyl radicals were the exclusive precursor species, their sticking probability, onto the growing film surface, could be determined. A methyl radical sticking probability consistent with this picture is 0.02 to 0.03 , calculated as the ratio of the deposition rate, in terms of the number of carbon atoms, to the methyl radical flux.

The methyl radical density estimated here can be compared to that recently reported by Toyoda et al[6], i.e., $4.7 \times 10^{11} \mathrm{~cm}^{-3}$ for an $\mathrm{rf}$ power of $25 \mathrm{~W}$ at $10 \mathrm{mTorr}$. In view of the lower pressure and rf power used by these authors, the value is in relatively good agreement with that estimated here. They also predicted that the sticking probability of the methyl radicals with the stainless steel walls of their system would be less than $10^{-3}$. This is also consistent with our results, as the sticking coefficient of methyl radicals with the energetic film surface may be higher than that for collisions with the chamber walls.

\section{Ions}

The total ion current measurement is of great significance with regard to possible growth mechanisms of a-C:H films. As discussed earlier, the measurement of total ion current made in this work is an upper limit to the real ion current. By comparing the measured total ion current to the calculated deposition rate of $\mathrm{C}_{1}$ from the previous section, it can be seen that the ion current is insufficient to account for this deposition rate. The total measured ion current at the powered electrode was $1.7 \times 10^{15} \mathrm{~cm}^{-2} \mathrm{~s}$. The calculated $\mathrm{C}_{1}$ deposition rate was $1.9 \times 10^{15} \mathrm{~cm}^{-2} \mathrm{~s}$. At the grounded electrode the measured ion current was $0.4 \times 10^{15} \mathrm{~cm}^{-2} \mathrm{~s}$, as compared to the calculated $\mathrm{C}_{1}$ species deposition rate of $1.0 \times 10^{15} \mathrm{~cm}^{-2} \mathrm{~s}$. In both cases the ion current is less than 
the deposition rate. Although the values are quite close, almost within experimental variation, the evidence still points quite strongly against ions being the predominant deposition species. A large fraction of the true ion current results from $\mathrm{H}^{+}$and $\mathrm{H}_{2}^{+}$ species, which do not contribute to the deposition rate. Also, the current doubling effect of secondary electrons makes the measurement of ion current an upper limit on the true ion current. Thirdly, the sticking coefficient of the deposition species may be equal to one only at the maximum theoretical limit. Realistically, it must be some value less than one. If ions were the predominant deposition species, the measured value for ion current would be expected to be significantly higher than the calculated $\mathrm{C}_{1}$ deposition rate.

The methyl radical flux is at least 40 times greater than the total ion flux. The total ion flux is insufficient for ions to be the predominant deposition species. Some contribution of ions to the total deposition rate has not been ruled out, but the results described in this chapter suggest that plasma radicals are responsible for the majority of the bulk mass deposition of the films. More studies are necessary, however, if the respective roles of ions and radicals in the growth mechanism of a-C:H films are to be elucidated. 


\section{Bibliography}

[1] K. Tachibana, M. Nishida, H. Harima, and Y. Urano, J. Phys. D 17, 1727 (1984).

[2] L.E. Kline, W.D. Partlow, and W.E. Bies. J. Appl. Phys. 65, 70 (1989).

[3] C.E. Melton and P.S. Rudolph, J. Chem. Phys. 47, 1771 (1967).

[4] Y. Catherine and P. Couderc, Thin Solid Films 144, 265 (1986).

[5] F.A. Baiocchi, R.C. Wetzel, and R.S. Freund, Phys. Rev. Lett. 53, 771 (1989).

[6] H. Toyoda, H. Kojima, and H. Sugai, Appl: Phys. Lett. 54, 1507 (1989). 


\section{Chapter 5}

\section{Plasma Control}

\subsection{Introduction}

The results up to this point suggest that the significant difference in the growth conditions between the powered and grounded electrodes is the relatively high flux and energy of the ions impinging on the rf powered electrode. Since the films formed at the powered electrode are mechanically superior to those formed at the grounded electrode, ion impact processes are likely the origin of the hardness of these films. However, the respective roles of plasma ions and radicals in a- $\mathrm{C}: \mathrm{H}$ film formation have yet to be elucidated.

In order to understand the growth mechanism of a- $\mathrm{C}: \mathrm{H}$ films, the roles played by both the plasma ions and the radicals must be determined. Ideally, ions and radicals must be studied independently. Achieving independent control of the ions and the radicals, in the complex chemical system of a plasma, is exceedingly difficult. Most work done in the past has utilized the effects that gas pressure and rf power have on the self bias voltage, and thus the ion energy. While ion energy can be controlled in this way, variations in $\mathrm{rf}$ power and gas pressure also affect the plasma density and thus will alter the radical flux onto the growing film surface. In previous work the radical flux was not measured, thus an uncontrolled variable was at work to complicate interpretations of results.

Explained in this chapter, are experiments which were designed to control the effects of plasma ions and plasma radicals independently. The first series of experiments employ a novel technique, called pulsed biasing, in order to gain control of the ion energy flux to the surface without affecting the radical flux significantly. In order 
to control the radical flux, radical scavenging experiments were carried out which were designed to selectively reduce the flux of radicals, while maintaining most of the ion flux. The results from these experiments shed new light on the complimentary roles of ions and radicals in the growth of a-C:H films, and their effects on the final physical properties of the films.

\subsection{Pulsed Biasing Effect}

A pulsed biasing experiment was devised to enhance and control ion bombardment in order to illuminate the effect of the ions more clearly. By pulsing the nonpowered electrode to large negative voltages during the deposition, the flux of the high energy ions incident on the sample surface is controlled. Thus, conditions at the nonpowered electrode are altered to become more like powered electrode conditions. This control, unlike the of power control conventionally used to vary ion energy, has a relatively small effect on the radical flux, at long as the pulse width and frequency are varied so that the nonpowered electrode is near ground potential most of the time. A similar technique, but with pulse heights from $10-100 \mathrm{keV}$, has been employed by Conrad and Castagna $[1]$ for ion implantation. The technique used here employs much lower pulse voltages, thus bombarding the surface with ions of much lower energies, not implanting the ions in the traditional sense.

\subsubsection{Ion Current Density and Ion Energy Flux}

The ion current density was measured for each of the pulsed biasing conditions so that variations in film properties could be correlated with the apparent ion energy flux. As will be seen in the following sections, the properties of the $\mathrm{a}-\mathrm{C}: \mathrm{H}$ films varied smoothly with the apparent total ion energy flux but not with individual ion energy. The current density measurements were made as described in the chapter 2. The values for current density thus obtained are an upper limit on the real ion current density values, due to the possibility of current doubling effects from sec ndary electron emission. Since these effects only proportionally alter the measured values, relative changes in ion current density are realistic. The values are used to investigate the variations in film properties with changes in ion current density and ion energy. 
The values of apparent ion current density for various pulsed biasing conditions are given in Fig. 5.1. As noted earlier, a large difference in ion flux exists between powered electrode and grounded electrode conditions. It is greater by about a factor of four at the powered electrode. As the voltages and frequencies of the pulsed biasing conditions are increased, the average ion current also increases somewhat, as also visible in Fig. 5.1. The effect is subtle, as the most vigorous pulsed biasing condition yields an ion current greater by a factor of less than two over the value measured for the grounded electrode conditions.

Since no temporal variations were observed for the ion current under the pulsed biasing conditions, the calculation of the average ion energy flux is relatively simple. It is given by the product of the measured D.C. current and the time averaged potential drop between the lower electrode and the plasma bulk. The values calculated in this way were used in the plots showing the variation of film properties with ion energy flux, as given in this chapter. The properties of the a-C:H films examined in this study, were found to vary smoothly with the ion energy flux, not with individual ion energy, hence the motivation for plotting the measurements versus ion energy flux. The noted variation of film properties with ion energy flux is dealt with in more detail in the discussion section.

\subsubsection{Hardness and Deposition Rate}

The thickness deposition rate of a- $\mathrm{C}: \mathrm{H}$ films was found to decrease with ion energy flux as displayed in Fig. 5.2, howe'er, the mass deposition rate was found to remain approximately constant. Therefore, it appears that the ions act to densify the films. The variation of Knoop hardness and compressive stress of a-C:H films as a function of ion energy flux is also shown in Fig. 5.2. The hardness and compressive stress of $\mathrm{a}-\mathrm{C}: \mathrm{H}$ films produced at the pulsed biased electrode were found to increase monotonically with the ion energy flux. As shown in the inset of Fig. 5.2, the curves can be extrapolated far down to the high energy flux values of hardness and compressive stress which were measured for the films formed at the powered electrode. Thus it can be seen that the mechanical properties of a-C:H films are controlled by the energy flux supplied to the film surface by ions but not on the individual ion energy. Also, 


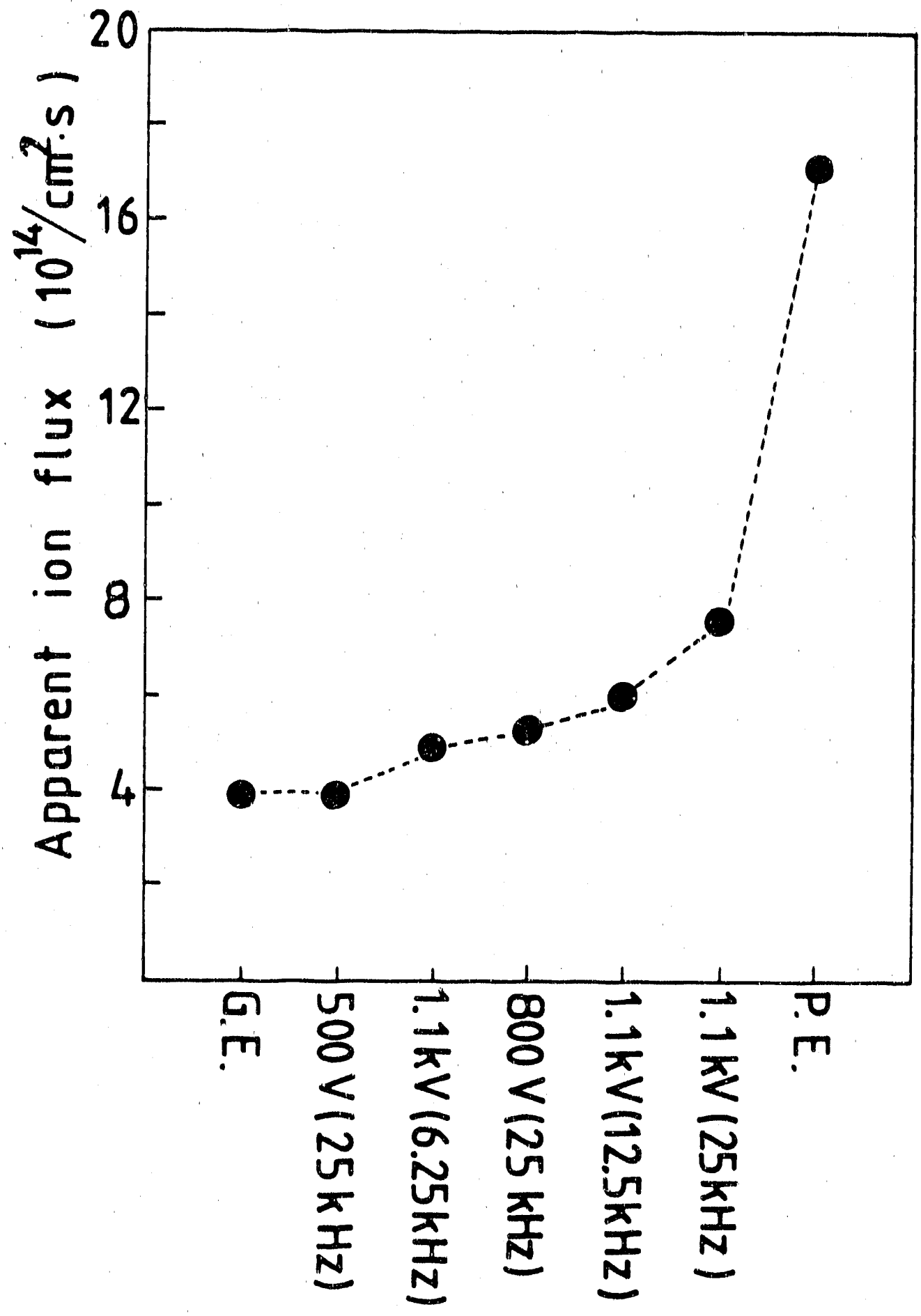

XBI 906-2127

Figure 5.1: Apparent ion flux on the substrate for various deposition conditions. 

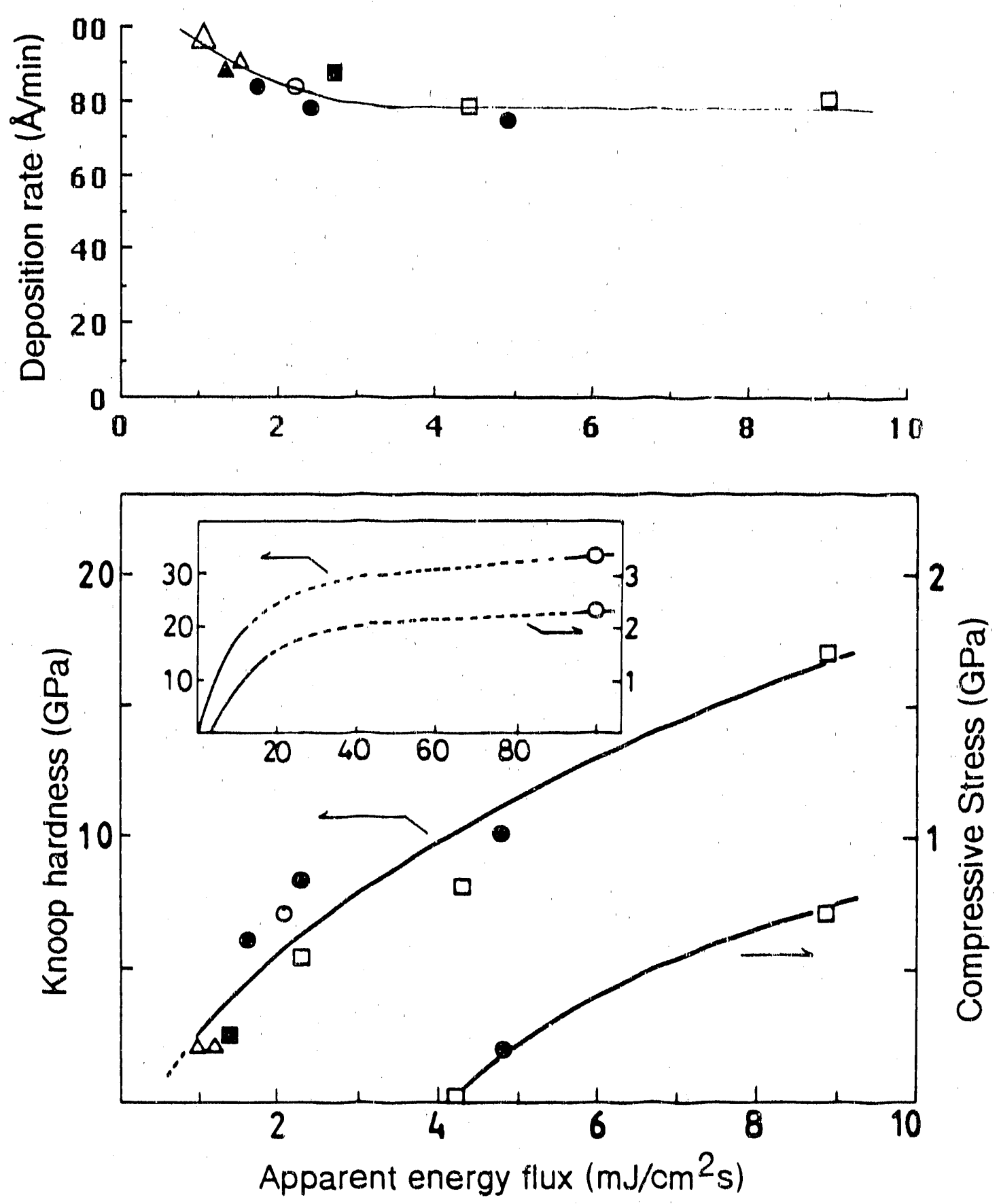

$X B I \quad 906-2134$

Figure 5.2: The upper plot shows the a-C:H deposition rate as a function of ion energy flux. In the lower plot, the hardness and compressive stress of the a-C:H films is plotted as a function of ion energy flux. 
the mass deposition rate of a-C:H films onto silicon substrates is not significantly affected by the ion energy flux.

\subsubsection{FTIR}

The FTIR spectra of the films were measured as a function of biasing conditions in order to investigate how the $\mathrm{C}-\mathrm{H}$ bonding in the films varied with energy flux. The concentrations of methylene $\left(-\mathrm{CH}_{2^{-}}\right)$and methyl $\left(-\mathrm{CH}_{3}\right)$ groups were determined from the FTIR spectra and are plotted versus energy flux in Fig. 5.3. The concentrations of both methylene and methyl groups in the films show a rapid decrease with the energy flux and level off at a relatively small value of energy flux of about $4 \mathrm{~mJ} \mathrm{~cm}^{-2} \mathrm{~s}^{-1}$ The methyl group concentration has decreased to near zero at this point, suggesting that most of the crosslinking, which acts to expand a three dimensional amorphous network, has been completed at this value of energy flux. It is interesting to note that it is at this same value of energy flux that the compressive stress of the films begins to increase, as can be seen in Fig. 5.2.

\subsubsection{Optical and Raman Spectra}

The optical gap of the a- $\mathrm{C}: \mathrm{H}$ films was measured as a function of biasing conditions. Fig. 5.4 shows the optical gap plotted as a function of ion energy flux. The optical gap was determined from the Tauc plot of the absorption coefficient using a linear extrapolation of the absorption edge[2]. The figure shows the optical gap decreasing rapidly and then leveling off, again at an energy flux of about $4 \mathrm{~mJ} \mathrm{~cm} \mathrm{~cm}^{-2} \mathrm{~s}^{-1}$.

Concurrent with the decrease of the optical gap, we also found that the Urback edge[3] (the optical absorption associated with localized states near the band edges) became less and less distinctive. The localized states are presumed to be at least partly associated with an incomplete three dimensional amorphous network, so the above trend is consistent with the rapid decrease of the $-\mathrm{CH}_{3}$ concentration as demonstrated in Fig. 5.3. The localized states can also serve as effective fluorescence centers in the a-C:H films[4]. In fact, a strong fluorescence background prevented the acquisition of Ram in spectra for films produced with an energy flux less than $2.3 \mathrm{~mJ} \mathrm{~cm}^{-2} \mathrm{~s}^{-1}$. Other films grown under higher energy flux, however, have shown Raman spectra 


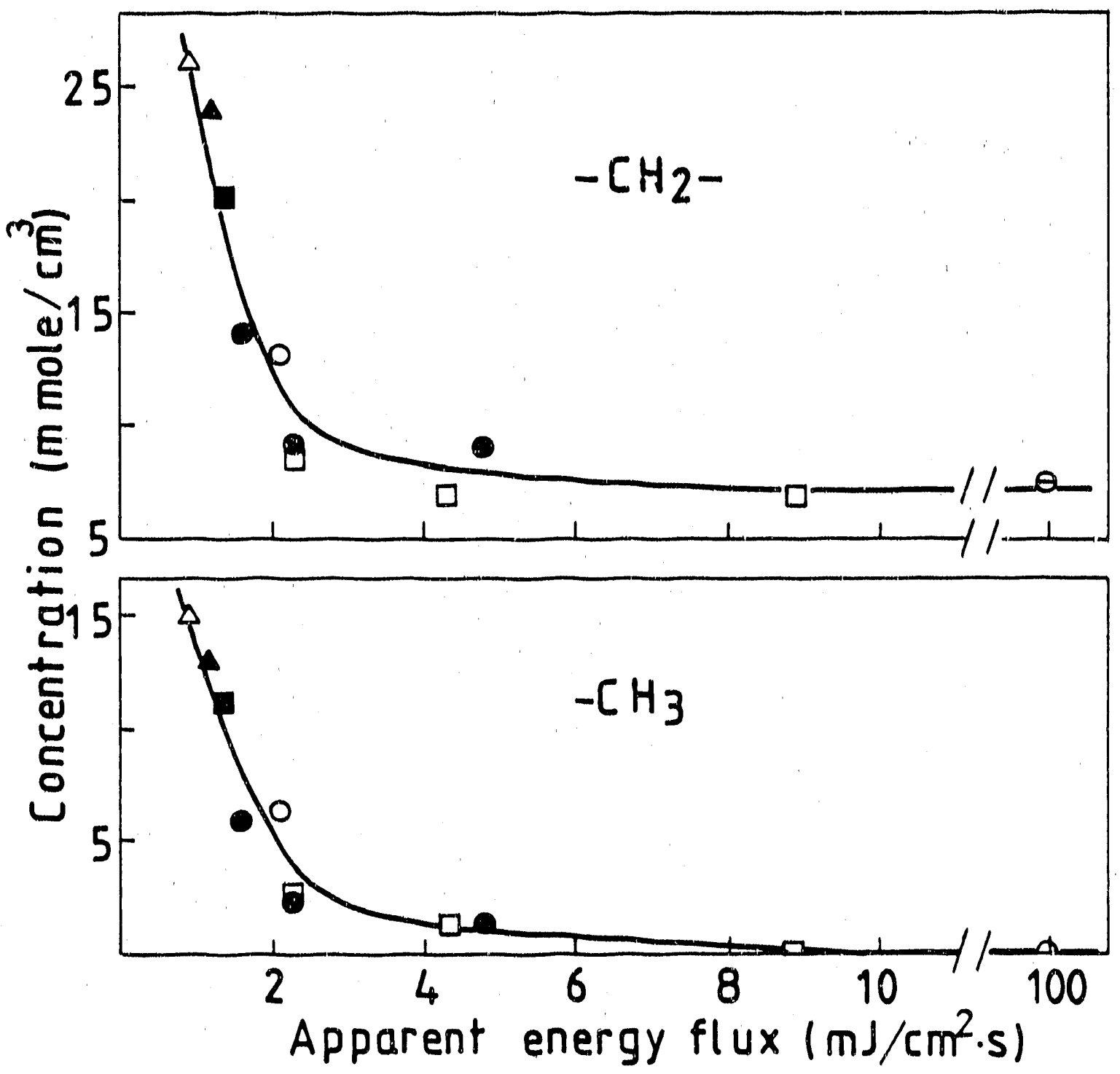

XBL $906-2126$

Figure 5.3: Concentrations of methylene $\left(-\mathrm{CH}_{2}-\right)$ and methyl $\left(-\mathrm{CH}_{3}\right)$ groups in a-C:H films as a function of ion energy flux. 


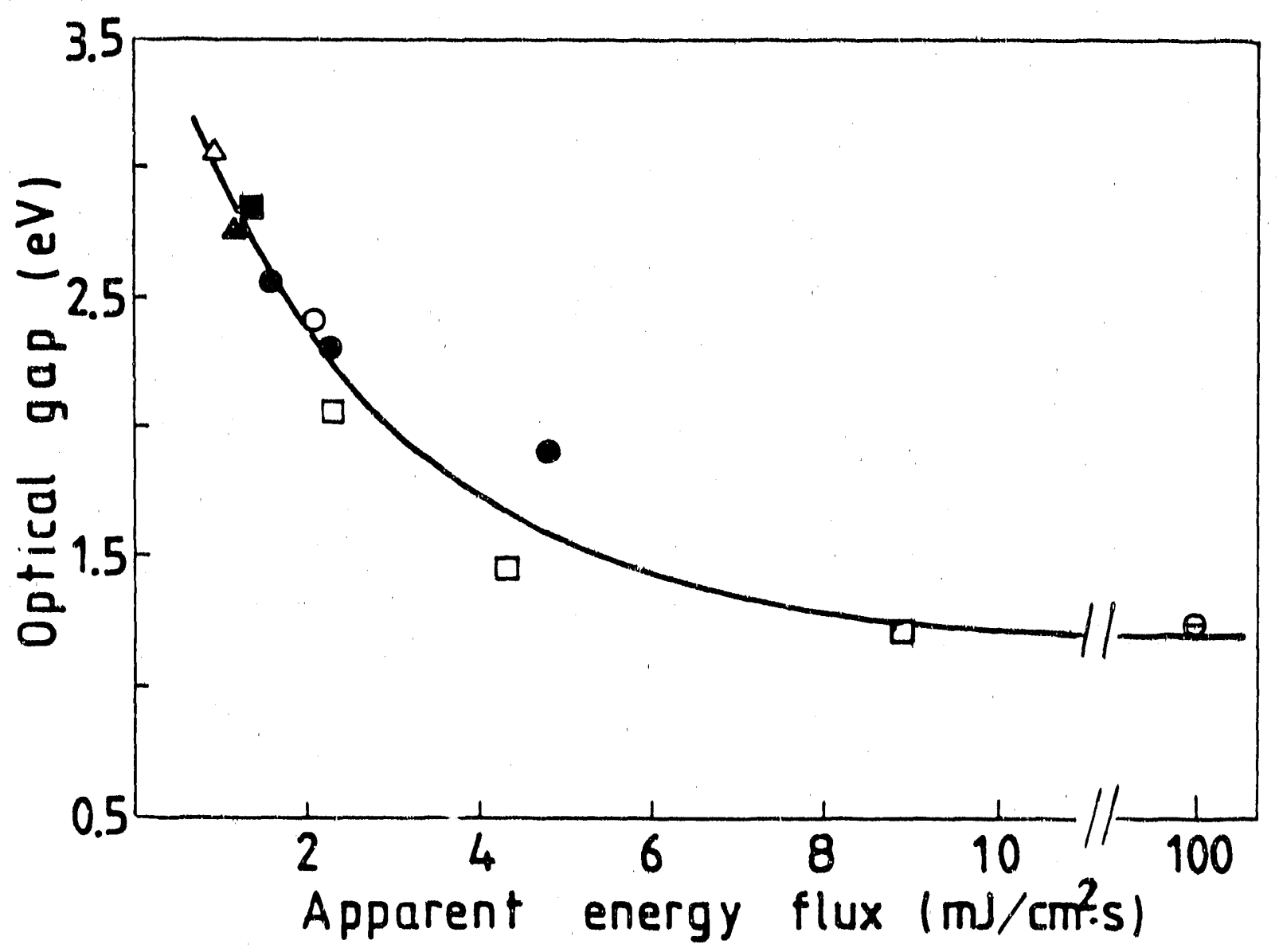

XBL $906-2125$

Figure 5.4: Changes in the optical gap of a-C:H films as a function of ion energy flux. 
characteristic of the amorphous carbon phase. The Raman spectra were not found to vary significant'y with ion energy flux or individual ion energy.

\subsection{Radical Scavenging}

The studies utilizing the pulsed biasing technique showed the hardening effect of ion energy flux, and the measurements of ion flux indicated that ions are not the primary deposition species. In order to determine the radical contribution to film growth, the effect of a radical scavenger on growth rates was studied. It has been demonstrated in the case of silicon growth by PACVD, that the addition of a small amount of a radical scavenger, such as NO, into the plasma causes a large decrease in the deposition rate[5]. Fig. 5.5 shows the effect of NO on the deposition rate of a$\mathrm{C}: \mathrm{H}$ films. Here the total gas pressure before plasma ignition was kept constant at 65 mTorr, as before, in order to avoid large changes of plasma parameters. Although this caused a decrease in methane pressure, it amounted to only $20 \%$ for the maximum NO content. Therefore this effect can account for only a moderate decrease in the deposition rate. In Fig. 5.5, a very strong decrease in growth rate is evident in the case of the powered electrode, where as little as $5 \%$ NO decreases the growth rate by more than a factor of two, and no deposition was observed in the presence of $20 \%$ NO. A decrease in growth rate is also measured for the film grown on the grounded electrode, however this decrease is much less rapid.

Auger electron spectroscopy was performed on a film formed at the grounded electrode with the addition of $10 \% \mathrm{NO}$ to the plasma. It was clear that a large amount of nitrogen and oxygen had been incorporated into the film formed on the grounded electrode. It is apparent that the product of radical scavenging, some sort of CNO polymer, was deposited at the grounded electrode. AES also demonstrated negligible NO incorporation into the film formed at the powered electrode. The additional presence of the CNO polymer at the grounded electrode accounts for the less rapid decrease in deposition rate there, as compared to the rate at the powered electrode. The CNO polymer is likely susceptible to ion bombardment, hence its absence at the powered electrode.

It is important to demonstrate that $\mathrm{NO}$ is not scavenging the ions as well as the 


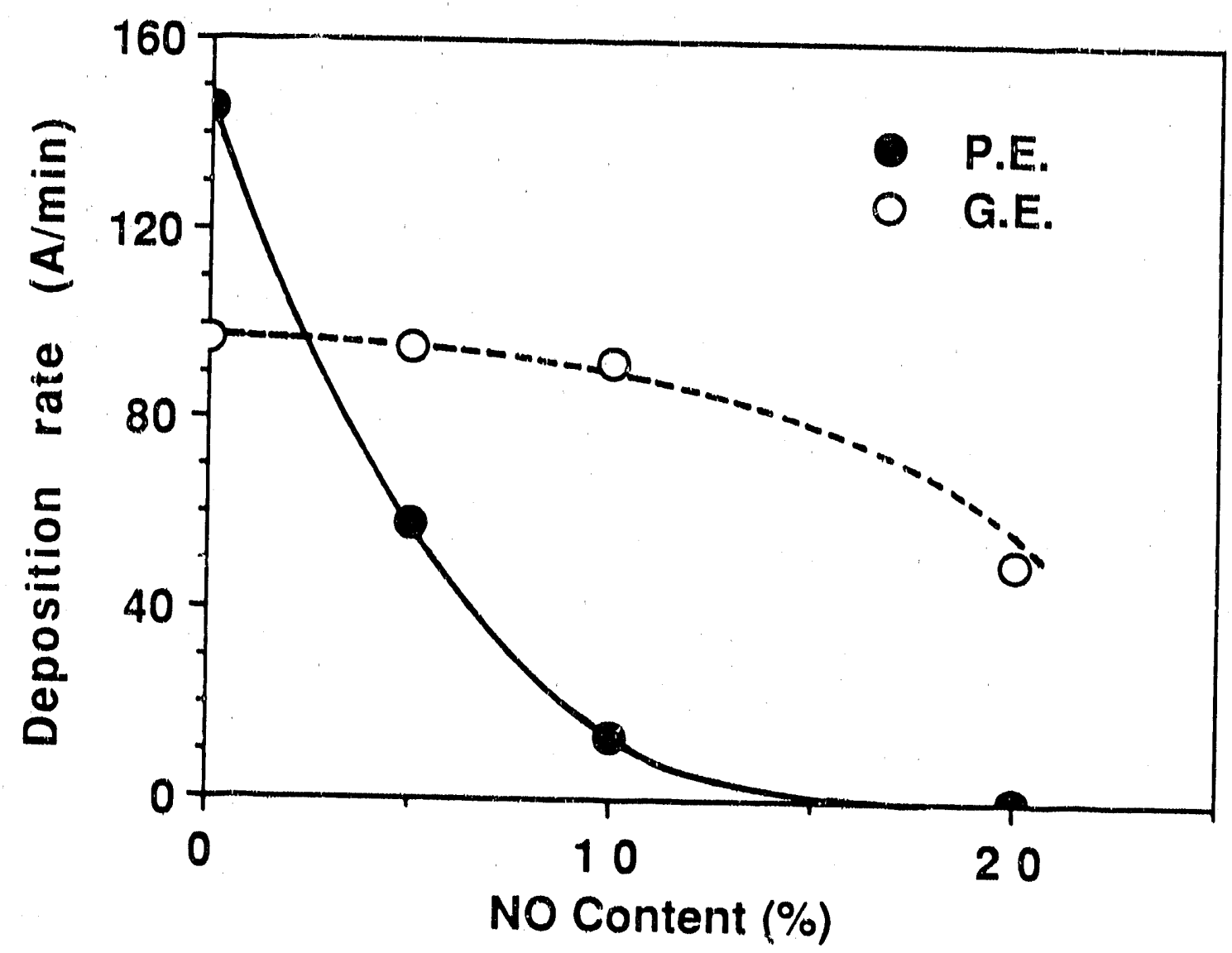

XBL $896-2528$

Figure 5.5: Effect of nitric oxide addition on the deposition rate at the PE and the GE. Radical scavenging by NO decreases the deposition rate dramatically. 
radicals. Fig. 5.6 shows the mass spectra of ions incident on the powered electrode with and without the addition of NO (20\%). The intensity of the ion signal has decreased by about a factor of two with the addition of $20 \%$ NO. However, as is seen in Fig. 5.6 the growth rate of the films has decreased to zero at this point. If the ions were the predominant precursor, we should still see a growth rate on the order of $80 \AA / \mathrm{min}$. Hence we can conclude that it is a radical scavenging effect, not an ion scavenging effect, that accounts for the decrease in growth rate by the addition of NO.

This radical scavenging study shows conclusively that radicals in the plasma must be the precursors responsible for the deposition of the majority of the film mass.'

\subsection{Discussion}

The experimental results described above demonstrate close correlations among various film properties. In the region of relatively low ion energy flux, the supplied energy seems to act to promote crosslinking between carbon chains to expand the three dimensional amorphous network. This is indicated by the rapid decrease in both methylene and methyl group concentrations as a function of ion energy flux in the region of relatively low flux. The structural network is not built up only with the $\mathrm{sp}^{3}$ bonding configuration, but is inevitably accompanied by the formation of $\mathrm{sp}^{2}$ clusters or $\pi$ bonded domains, which account for the decrease of the optical gap[6], as seen in Fig. 5.4. The presence of small domains of graphite-like structure at the surface of a-C:H films has also been observed by scanning tunneling microscopy[7](see chapter 7). The expansion of the three dimensional network also results in a significant increase in hardness. However, in this region of relatively low ion energy flux, and in association with the resultant higher hydrogen content, the films are probably more deformable, resulting in a relief of any compressive stress which might build up. As a result, the compressive stress remains below our limit of detection up to a hardness

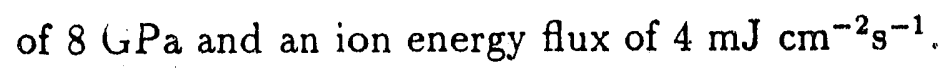

In the ion energy flux range greater than abcut $4 \mathrm{~mJ} \mathrm{~cm}^{-2} \mathrm{~s}^{-1}$, almost no change in methylene group concentration or optical gap is seen, and the methyl group concentration falls close to zero. Nevertheless, the hardness continues to increase considerably, 


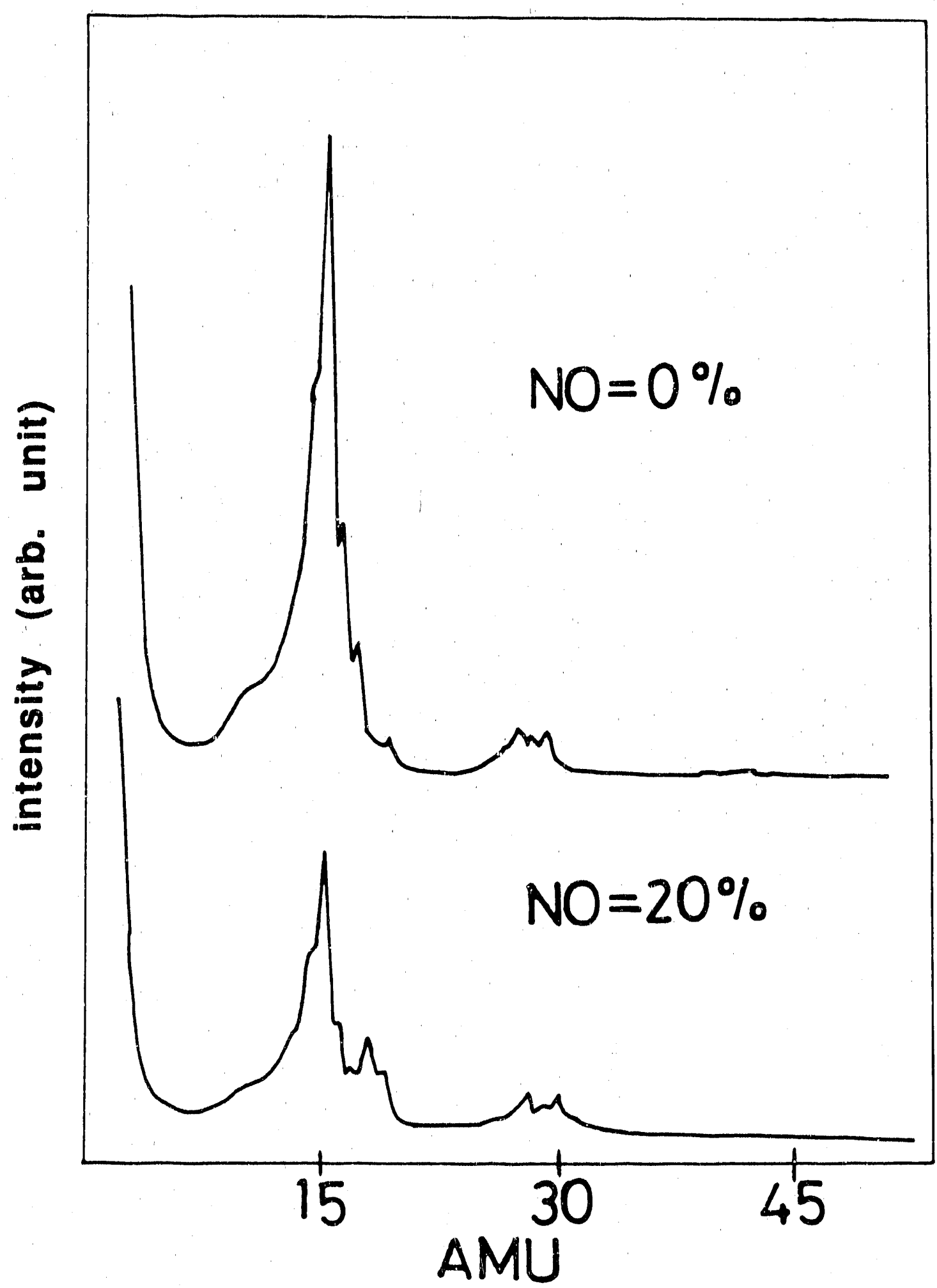

Figure 5.6: Mass spectra of ions incident on the powered electrode for $0 \%$ NO feedgas concentration and for $20 \% \mathrm{NO}$. The presence of the radical scavenger has a relatively small effect on ion flux. 
from about $15 \mathrm{GPa}$ up to more than $30 \mathrm{GPa}$. Since the crosslinking seems to have been mostly completed already at lower energy flux values, the factor remaining which could account for the hardening is a densification of the films. In fact, the density of the film grown on the pulsed biased electrode with a flux of about $4 \mathrm{~mJ} \mathrm{~cm}^{-2} \mathrm{~s}^{-1}$, was approximately $1.4 \mathrm{~g} / \mathrm{cm}^{3}$, still less than that of films produced on the rf powered electrode (approx. $1.7 \mathrm{~g} / \mathrm{cm}^{3}$ ). The deformability of the material is probably less at these high ion energy flux values, so the compressive stress now begins to increase in parallel with the hardness.

The radical scavenging study demonstrated how plasma radicals are responsible for the bulk of the mass deposition of the $\mathrm{a}-\mathrm{C}: \mathrm{H}$ films. The ions act to alter the structure of the material being deposited. The properties of the films vary with ion energy flux but not with the energy of the individual ion. This suggests a mechanism involving large but local thermal spikes quenched rapidly by the bulk of the film, as proposed by Weissmantel[8]. Within these energetic spike regions, various processes - bond rearrangement, cross linking accompanied by hydrogen elimination, partial graphitization, etc. - could be highly activated. Also, a rapid quenching of the energetic surface layer by the bulk of the film is likely to be related to the high residual compressive stress, as nonequilibrium structures could be frozen out of the energetic surface. 


\section{Bibliography}

[1] J.R. Conrad and T. Castagna, Bull. Am. Phys. Soc. 31, 1479 (1986).

[2] U.J. Tauc, R. Grigonovici, and A. Vancu, Phys. Status Solidi 15, 627 (1966).

[3] T. Datta, J.A. Woolam, and W. Notohamiprodjo, Phys. Rev. B 40, 5956 (1989).

[4] J. Gonzalez-Hernandez, B.S. Chao, and D.A. Pawlik, J. Vac. Sci. Tech. A 7', 2332 (1989).

[5] P.A. Longeway, R.D. Estes, and H.A. Weakliem, J. Phys. Chem. 88, 73 (1984).

[6] M.A. Tamor, J.A. Haire, C.H. Wu, and K.C. Haas, Appl. Phys. Lett. 54, 123 (1989).

[7] G.J. Vandentop, P.A.P. Nascente, M. Kawasaki, D.F. Ogletree, G.A. Somorjai, and M. Salmeron, submitted J. Appl. Phys.

[8] C. Weissmantel, J. Vac. Sci. Tech. 18, 179 (198I). 


\section{Chapter 6}

\section{Interface Characterization}

\subsection{Introduction}

Characterization of the interface between the a-C:H film and the substrate is most important for the potential applications of the films as protective coatings. Hard a$\mathrm{C}: \mathrm{H}$ films, produced under high energy ion impact, exhibit a large compressive stress of several $\mathrm{GPa}[1]$, so good adhesion to the substrate is a prerequisite to prevent delamination. The films exhibit especially good adhesion characteristics when deposited on single crystal silicon substrates. Hard a-C:H films more than $2 \mu \mathrm{m}$ thick are easily grown on silicon without any delamination. The films are not noticeably damaged nor delaminated by a scratch adhesion test with an $830 \mathrm{~g}$ load[1]. This strong adhesion of a-C:H films on silicon suggests that the films are bound to silicon by strong chemical bonds, and that a silicon carbide layer is likely to be present at the interface.

Sander and coworkers have used depth profiling with argon ion sputtering in their study using XPS and AES to identify the interfacial carbide[2]. They proposed a carbide layer extending over nearly a hundred $\AA$, not only for hard a-C:H films on silicon, but also for soft polymer-like films that showed poor adhesion. It is possible, however, that the high energy ion depth profiling used induced a structural change at the interface of the material being studied. Mixing occurs as a result of the strong forward momentum transfer from the sputtering ions to target atoms. Such mixing could result in an artificially broad measurement of the interface thickness.

In this chapter, the results of an XPS analysis of the silicon-carbon film interface without the use of ion sputtering are presented. The a-C:H films studied in this work were deposited to thicknesses where the XPS signal originating from the interface 
region still contributed significantly to the overall spectrum. Due to the short mean free path of the photoelectrons, the contribution of surface (or interface) atoms can be enhanced relative to that from the underlying substrate atoms, by changing the angle of collection of the photoelectrons. The existence of silicon carbide at the interface of a hard a-C:H film grown on silicon was confirmed. However, the thickness of the carbide as determined from angle-resolved spectra was much smaller than that reported by Sander and coworkers. Moreover, there was no carbide formation evident at the interface of a soft polymer-like film on silicon. Films formed at varying self bias voltages and pulsed biasing conditions were studied in order to determine the effect of ion energy on interface thickness. The thickness of the the $\mathrm{SiC}$ interface was observed to increase with ion bombardment energy. It was concluded that ion bombardment during a-C: $\mathrm{H}$ deposition contributes to strong adhesion by inducing the formation of an interfacial carbide layer.

\subsection{Angle-resolved XPS}

\subsubsection{Interface Characterization}

To analyse interfacial composition [3], XPS spectra in the regions of interest were taken as a function of takeoff angle for a series of short deposition times. Fig. 6.1 shows the silicon $2 \mathrm{p}$ spectra at two of the takeoff angles $\left(60^{\circ}\right.$, and $\left.15^{\circ}\right)$ for $0 \mathrm{~s}, 5 \mathrm{~s}$, and $20 \mathrm{~s}$ deposition times on the powered electrode $(50 \mathrm{~W})$, where a hard (about $30 \mathrm{GPa}$ ) a- $\mathrm{C}: \mathrm{H}$ film is produced. The uppermost two spectra, corresponding to zero a-C:H deposition tirne, were taken with a control substrate that had received the identical treatment as had other samples, excluding plasina ignition. These control Si $2 p$ spectra did not exhibit any oxide peak even near the grazing takeoff angle of $15^{\circ}$. Furthermore, the peak shape was identical with that for spectra obtained from silicon wafers that had been first etched in HF and further sputter cleaned by argon ions. Oxide formation on the HF treated silicon wafers from exposure to background gases in the plasma chamber, therefore, can be ruled out.

The hard a-C:H deposition on silicon introduced a higher binding energy shoulder in the Si $2 \mathrm{p}$ spectra. The intensity of this shoulder relative to the pure $\mathrm{Si} 2 \mathrm{p}$ peak increased as the photo-electron take-off angle was reduced (see Fig. 6.1). Fig. 6.1 

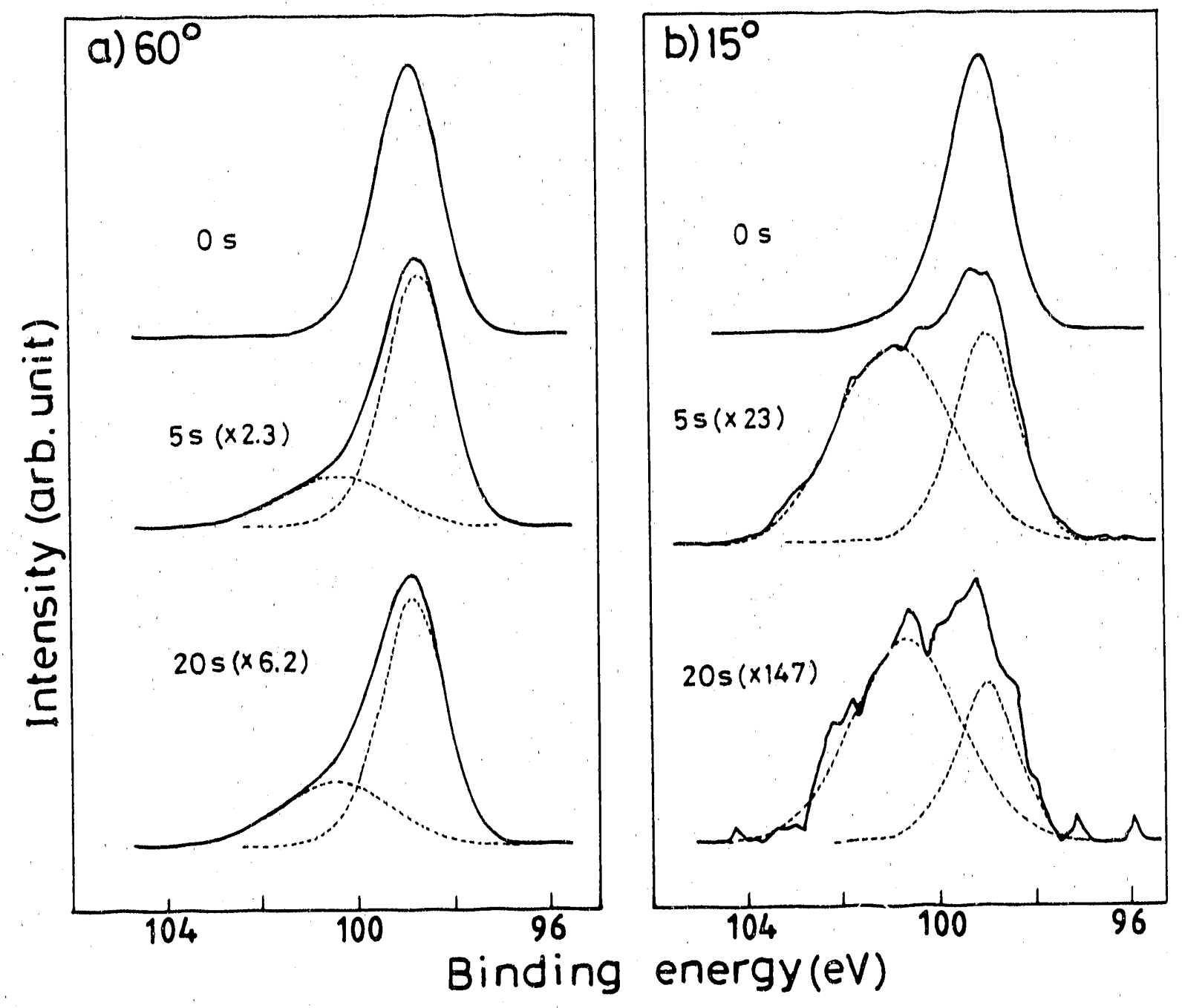

XBL 898-3042

Figure 6.1: Si 2p spectra for various deposition times on $\mathrm{Si}(100)$ placed on the powered electrode, where a hard a- $\mathrm{C}: \mathrm{H}$ film is produced. (a) electron takeoff angle $=60^{\circ}$. (b) electron takeoff angle $=15^{\circ}$. 
also shows the results of a computer-assisted separation of the $\mathrm{Si} 2 \mathrm{p}$ spectra into two overlapping peaks, i.e., a pure $\mathrm{Si} 2 \mathrm{p}$ peak due to the substrate bulk, and a higher binding energy component associated with the interface. A set of curve fitting parameters for the pure Si $2 \mathrm{p}$ signal from the substrate was obtained by analyzing the control spectra of films with no a-C:H deposited. These parameters were used in the peak separation of spectra where secondary interfacial contributions were present.

It can be seen in Fig, 6.1 that the intensity ratio of the higher binding energy peak to the pure Si 2 p peak depends strongly on the electron takeoff angle, but not on the deposition time in the range longer than $5 . \mathrm{s}$ (see also Fig. 6.5.) The deposition time, however, strongly affects the absolute intensity of the $\mathrm{Si} 2 \mathrm{p}$ signal. 'The angular dependance shows that the higher binding energy peak is associated with the $\mathrm{Si}$ atoms nearest the surface, ie., the silicon atoms at the substrate-film interface. The independence of the intensity ratio and the decrease in the absolute intensity with deposition times greater than 5 seconds, indicates that the interface formation is likely completed in the early seconds and is simply being covered up by a-C:H during the seconds thereafter.

The interfacial peak maxima were found between 100 and $101 \mathrm{eV}$, in agreement with the chemical shift of $\mathrm{Si} 2 \mathrm{p}$ state in silicon carbide reported by Wheeler and Pepper[4]. The identification of the interfacial layer as silicon carbide is supported by the concurrent change of $\mathrm{C}$ 1s spectra as shown in Fig. 6.2.

These spectra can also be separated into two overlapping peaks, one associated with the deposited a-C:H and a secondary peak at lower binding energy. The curve fitting parameters for the non-interfacial $\mathrm{C}$ 1s signal were obtained from a spectrum taken of an a-C:H film deposited for more than $40 \mathrm{~s}$, where the interfacial contribution to the spectrum is negligible. The maximum of the peak associated with the interface is at $283 \mathrm{eV}$, as expected for silicon carbide[2, 4].

Information about the stoichiometry of the interfacial carbide can be extracted from the intensity ratios of the $\mathrm{Si}$ and $\mathrm{C}$ signals associated with the interface. Fig. 6.3 shows a linear relationship between the peak areas of the $\mathrm{Si} 2 \mathrm{p}$ and $\mathrm{C} 1 \mathrm{~s}$ signals that were attributed to the interfacial carbide. The data were collected for various deposition times and takeoff angles. The line drawn in Fig. 6.3 with a slope of unity 


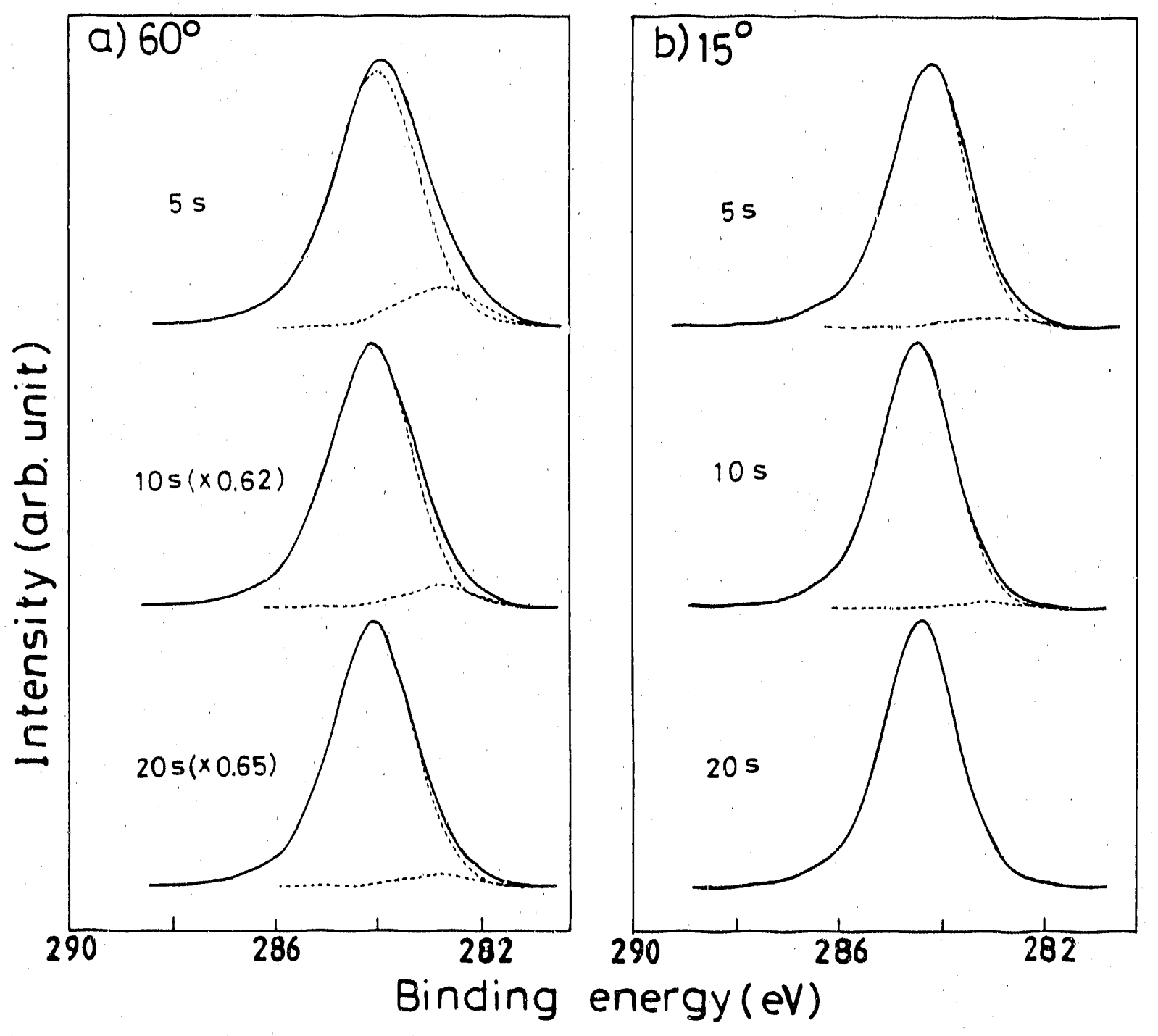

XBL $898-3043$

Figure 6.2: $\mathrm{C}$ 1s spectra for various deposition times on $\mathrm{Si}(100)$ placed on the powered electrode. (a) electron takeoff angle $=60^{\circ}$. (b) electron takeoff angle $=15^{\circ}$. 


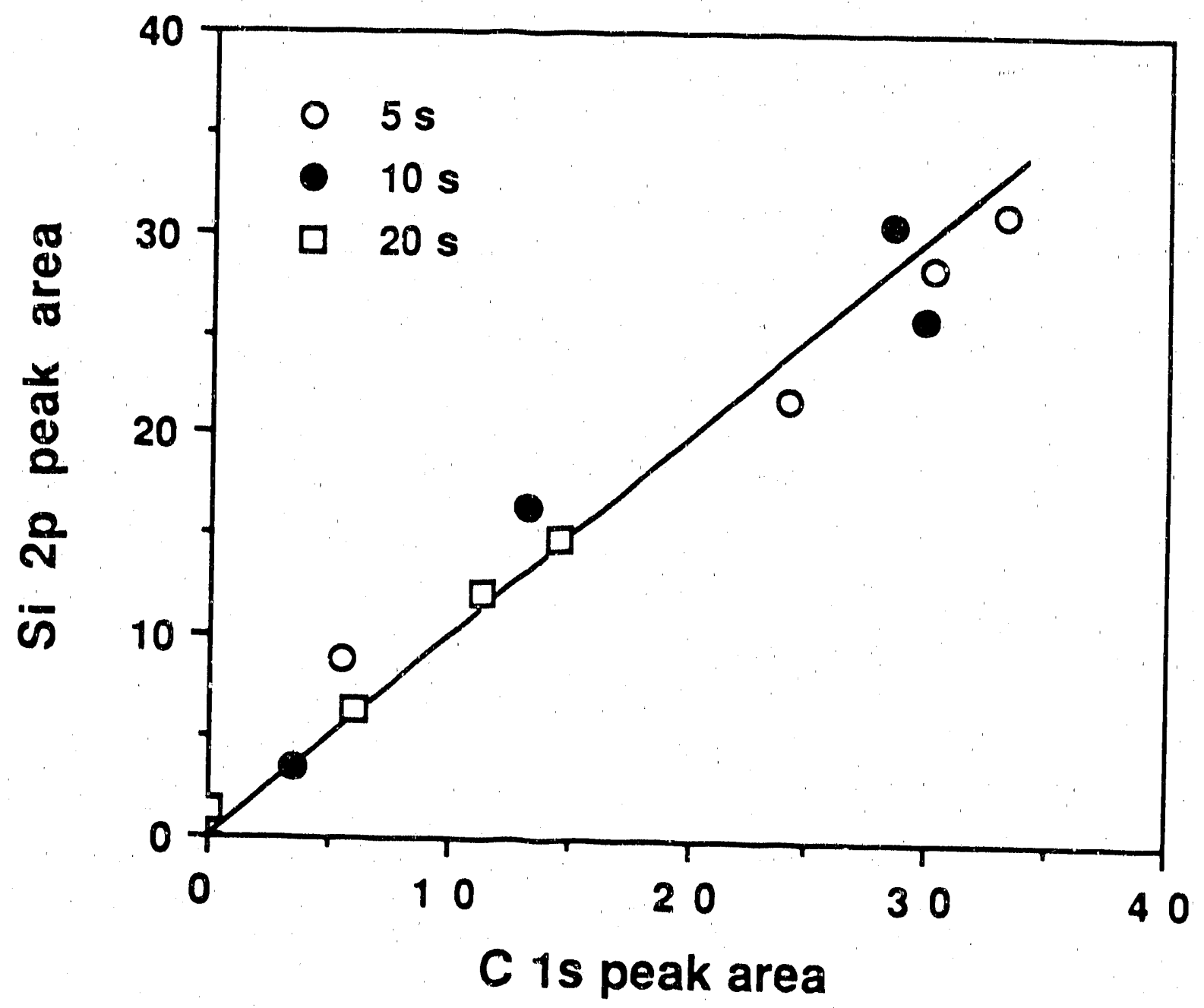

XBL $898-3044$

Figure 6.3: Relationship between the peak areas of Si 2p and C.1s signals associated with the interfacial silicon carbide layer. The data are collected for various deposition times $\left(5\right.$ to $20 \mathrm{~s}$ ) and electron takeoff angles $\left(60^{\circ}\right.$ to $\left.15^{\circ}\right)$. The intensity ratio of near unity suggests the silicon carbide layer is approximately stoichiometric. 
illustrates how closely the data fits a $1: 1$ intensity ratio. The sensitivity factors of the PHI 5300 XPS system for Si $2 \mathrm{p}$ and C 1s electrons are both equal to 0.25 . Also, the elastic mean free paths of photoelectrons in the a-C:H overlayer are expected to be about the same for the two peaks, because the difference in electron kinetic energy, $970 \mathrm{eV}$ for $\mathrm{C}$ 1s vs. $1155 \mathrm{eV}$ for Si $2 \mathrm{p}$, should cause only about a 10 percent difference in the mean free path[4]. As a result, the area intensity ratio of $\mathrm{Si} 2 \mathrm{p}$ to $\mathrm{C} 1 \mathrm{~s}$ of approximately $1: 1$ implies that an approximately stoichiometric silicon carbide layer has been formed at the interface.

In addition to $\mathrm{Si} 2 \mathrm{p}$ and $\mathrm{C}$ 1s, the XPS spectra also exhibited noticeable $\mathrm{O} 1 \mathrm{~s}$ intensity. Nonstoichiometric silicon oxide can also cause a higher binding energy shoulder in Si 2p spectra[4]. However, the good correlation between the Si $2 p$ and C 1s spectra in terms of chemical shift and peak area excludes any significant contribution from such oxide. Fig. 6.4 shows spectra in the regions of $\mathrm{O} 1 \mathrm{~s}, \mathrm{Si} 2 \mathrm{~s}$, and Si $2 \mathrm{p}$ peaks, which also demonstrates that the higher binding energy $\mathrm{Si} 2 \mathrm{p}$ shoulder is not associated with oxygen. Si related peaks are almost completely attenuated at a low takeoff angle of $15^{\circ}$. The $\mathrm{O}$ 1s signal intensity (the peak binding energy was between 531 and $532 \mathrm{eV}$ ) and its angle dependence varied from sample to sample, but in a typical case shown in Fig. 6.4, the $\mathrm{O}$ 1s peak does not suffer any significant intensity change as a function of takeoff angle. The PHI sensitivity factor for the $O$ is state is 0.66 , greater by factor of 2.6 than that for the $\mathrm{Si} 2 \mathrm{p}$ state. If the higher binding energy component in the Si $2 \mathrm{p}$ spectra was due to a suboxide of silicon, the $\mathrm{O}$ 1s signal in Fig. 6.4 would have to originate entirely from the interface. The insensitivity of the $O$ 1s peak intensity to takeoff angle excludes this possibility. The oxygen is probably due primarily to a small residual bulk and/or surface contamination of the a-C:H films.

The effective thickness of the interfacial carbide can be estimated from the angle dependence of the ratio of the interfacial Si $2 p$ intensity to the pure Si $2 p$ intensity from the substrate bulk, based on the relevant intensity attenuation equations[4]. In the presence of an a-C:H overlayer of thickness $d_{0}$ and an interfacial carbide of thickness $d_{i}$, the pure Si $2 \mathrm{p}$ intensity from the silicon substrate at takeoff angle $\theta$ can 


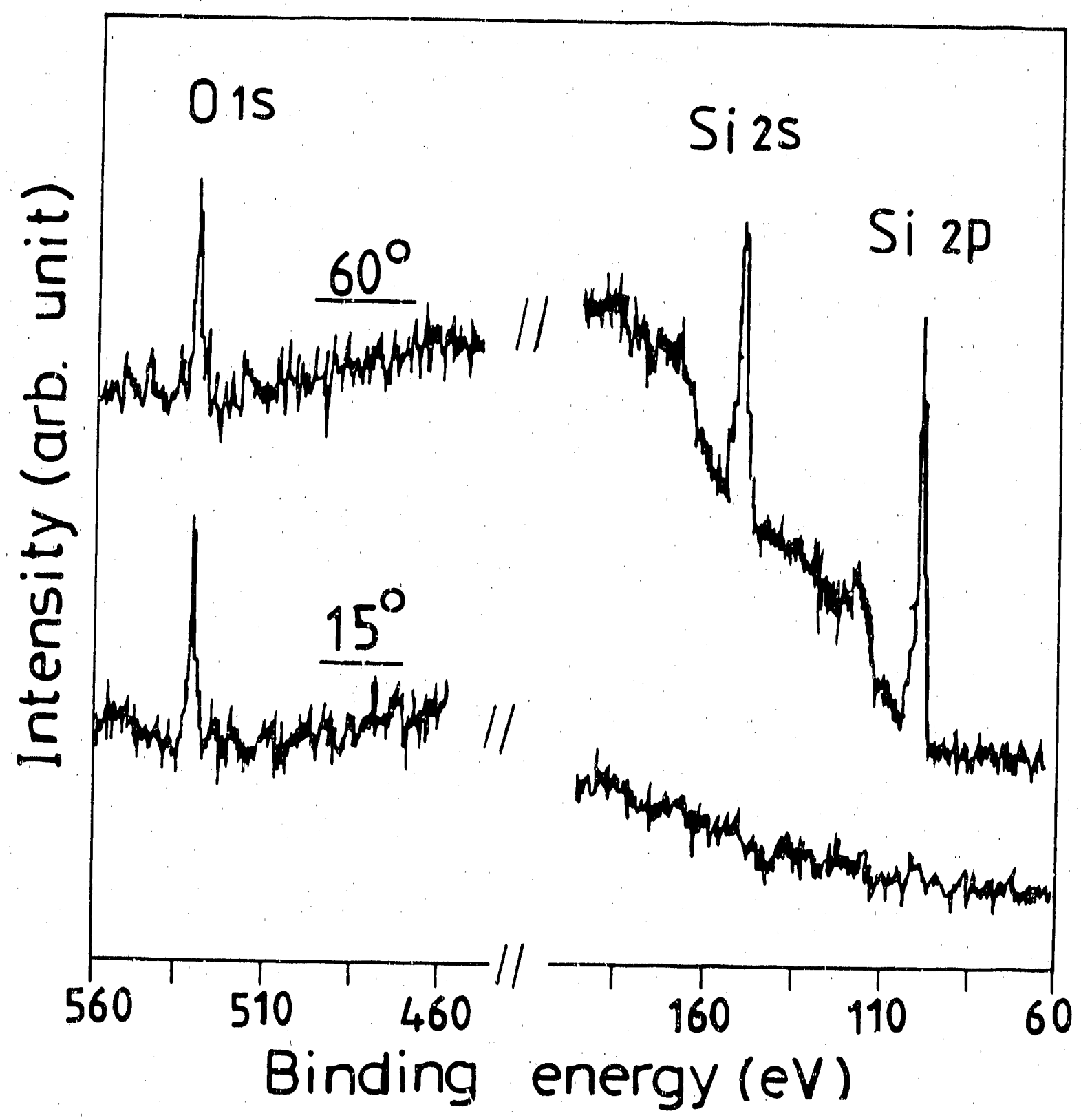

XBI 906-2133

Figure 6.4: XPS spectra showing the $\mathrm{O} 1 \mathrm{~s}, \mathrm{Si} 2 \mathrm{~s}$, and Si $2 \mathrm{p}$ peaks at electron takeoff is normalized to the $60^{\circ}$ and $15^{\circ}$. Deposition time is $20 \mathrm{~s}$ on the powered electrode. The intensity is normalized to the $\mathrm{C} 1 \mathrm{~s}$ peak to account for the angle dependant instrument factor. with the interface. 
be clescribed as $I_{s}(\theta)$, where

$$
I_{s}(\theta)=I_{\theta} \lambda_{s} \rho_{s} \exp \left(\frac{-d_{i}}{\lambda_{i} \sin \theta}\right) \exp \left(\frac{-d_{o}}{\lambda_{o} \sin \theta}\right)
$$

and $\lambda_{s}, \lambda_{i}$, and $\lambda_{0}$ are the elastic mean free paths in the silicon, the interfacial cartide, and the a-C:H overlayer, respectively, and $\rho_{s}$, represents the density of $\mathrm{Si}$ in the substrate. The factor $I_{6}$ in equation 6.1 accounts for the photoelectric emission cross section and the angle and energy dependent instrument factor. The Si $2 p$ intensity associated with the interfacial carbide is

$$
I_{i}(\theta)=I_{\theta} \lambda_{i} \rho_{i}\left[1-\exp \left(\frac{-d_{i}}{\lambda_{i} \sin \theta}\right)\right] \exp \left(\frac{-a_{o}^{\prime}}{\lambda_{o} \sin \theta}\right)
$$

where $\rho_{i}$ expresses the density of $\mathrm{Si}$ in the interfacial carbide. From equations 6.1 and 6.2 the ratio of $\mathrm{Si}<\mathrm{p}$ iritensity from interfacial silicon cartide to $\mathrm{Si} 2 \mathrm{p}$ intensity from the silicon substrate is given by

$$
\frac{I_{i}(\theta)}{I_{s}(\theta)}=\frac{\lambda_{i} \rho_{i}}{\lambda_{s} \rho_{s}}\left[\exp \left(\frac{d_{i}}{\lambda_{i} \sin \theta}\right)-1\right]
$$

which is independent of the parameters relating to the a-C:H overlayer as expected. Eq. 6.3 can be rewritten in a more convenient form like:

$$
R(\theta) \equiv \ln \left(1+\frac{\lambda_{s} \rho_{s} I_{i}(\theta)}{\lambda_{i} \rho_{i} I_{s}(\theta)}\right)=\left(d_{i} / \lambda_{i}\right)(1 / \sin \theta)
$$

Thus by plotting $R(\theta)$ as a function of $1 / \sin \theta$, one can determine the interfacial carbije thickness $d_{i}$ from the slope $d_{i} / \lambda_{i}$. The unknown parameters are $\rho_{i}$ and $\lambda_{i}$, which are for the interfacial carbide. Fig. 6.3 suggests the formation of approximately stoichiometric silicon carbide, so the paraneters for a single crystal $\mathrm{SiC}$ were used as a first approximation. Then $\rho_{s}=2.33 \mathrm{gcm}^{-3}[5], \rho_{i}=2.25 \mathrm{gcm}^{-3}$ (from the density of $\mathrm{SiC}, 3.21 \mathrm{gcm}^{-3}[2], \rho_{i}=\rho_{S i C}\left(\mathrm{~m}_{S i} /\left(\mathrm{m}_{S i}+\mathrm{m}_{C}\right)\right), \lambda_{s}=23 \AA[6]$, and $\lambda_{i}=15.6 \AA$ [4], respectively. Fig. 6.5 shows a plot of $R(\theta)$ as a function of $1 / \sin \theta$ for various depcisition times, based on the values given above. The fit to the linear function of $\mathrm{Eq} .6 .4$ is shown in the figure. The $5 \mathrm{~s}$ deposition gave a slightly lower slope, indicating that the interface formation is not strictly completed at this point. The data points for deposition times greater than $10 \mathrm{~s}$ can be fitted well by a single line, and give a slope $\left(d_{i} / \lambda_{i}\right)$ of $0.42 \pm 0.02$ so that $d_{i}$ is $6.5 \pm 0.3 \AA$, approximately $7 \AA$. 


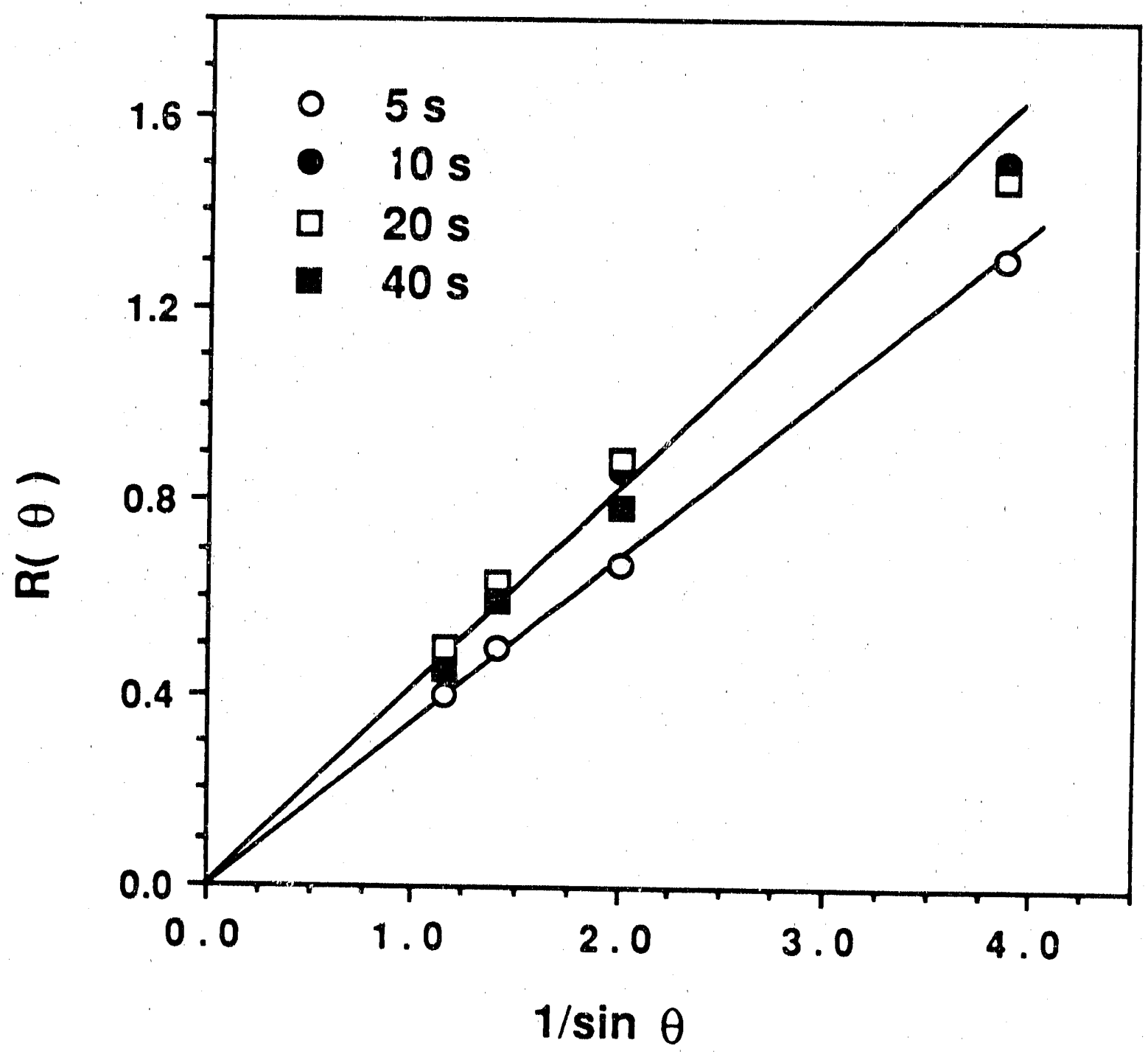

XBL $898-3045$

Figure 6.5: Dependence of $R(\theta)$ as defined in the text from the ratio of the chemically shifted $\mathrm{Si} 2 \mathrm{p}$ peak (from $\mathrm{SiC}$ ) to the pure $\mathrm{Si} 2 \mathrm{p}$ peak (from the $\mathrm{Si}$ substrate), on $1 / \sin \theta$. $\theta$ represents the electron takeoff angle. 
The interfacial carbide thickness oi $7 \AA$ determined above is significantly less than that suggested by Sander and coworkers[2]. The accuracy of the thickness determined here depends of course on the accuracy of the numerical parameters assumed. The calculations also assume that the materials behave as a stack of ideal uniform slabs. However, it appears unlikely that the carbide thickness could be more than an order of magnitude greater than the obtained value, as suggested by Sander and coworkers.

Unlike the hard a-C:H, a soft polymer-like film produced on the gruunded electrode exhibits not only a low hardness (about $2 \mathrm{GPa}$ ) but also poor adhesion, as judged by scratch adhesion tests. Thus the interface of the soft carbon film with the silicon substrate is expected to be considerably different from that of the hard a-C:H film. Fig. 6.6 shows a typical Si 2p spectrum for the soft polymer-like film ai a takeoff angle of $15^{\circ}$. The secondary peak maximum is located at about $101.8 \mathrm{eV}$, too high in er ergy to be attributable to silicon carbide. The energy is more characteristic of a suboxide of silicon[4]. It should be noted that no such oxide peak was observed in the spectra of the control substrates which were not subjected to the plasma discharge. This suggests that the oxide layer was formed by the action of the plasma, possibly due to oxygen containing impurity species such as water related ions and radicals. As can be seen from the spectrum in Fig. 6.6, taken near the grazing angle, the intensity

of the oxide peak is extremely small. A similar calculation as used to determine the interfacial carbide thickness resulted in a thickness of roughly $1 \AA$, which indicates that an interfacial monolayer does not exist.

\subsubsection{Effect of Ion Energy}

\section{RF Powur Dependence of Interface Thickness}

In order to investigate the effect of ion energy on interface thickness, the above procedures for determining $\mathrm{SiC}$ interface thickness were carricd out on a series of samples deposited at varying if power levels. In an rf plasma system, the self bias voltage of the powered electrode can be controlled by varying the rf power, as shown in Fig. 2.3. One drawback to this method of kinetic energy control is the fact that the plasma density and ion and radical flux to the substrate, also vary significantly with $\mathrm{rf}$ power. The $\mathrm{rf}$ power dependence of the interfacial thickness has, however, 


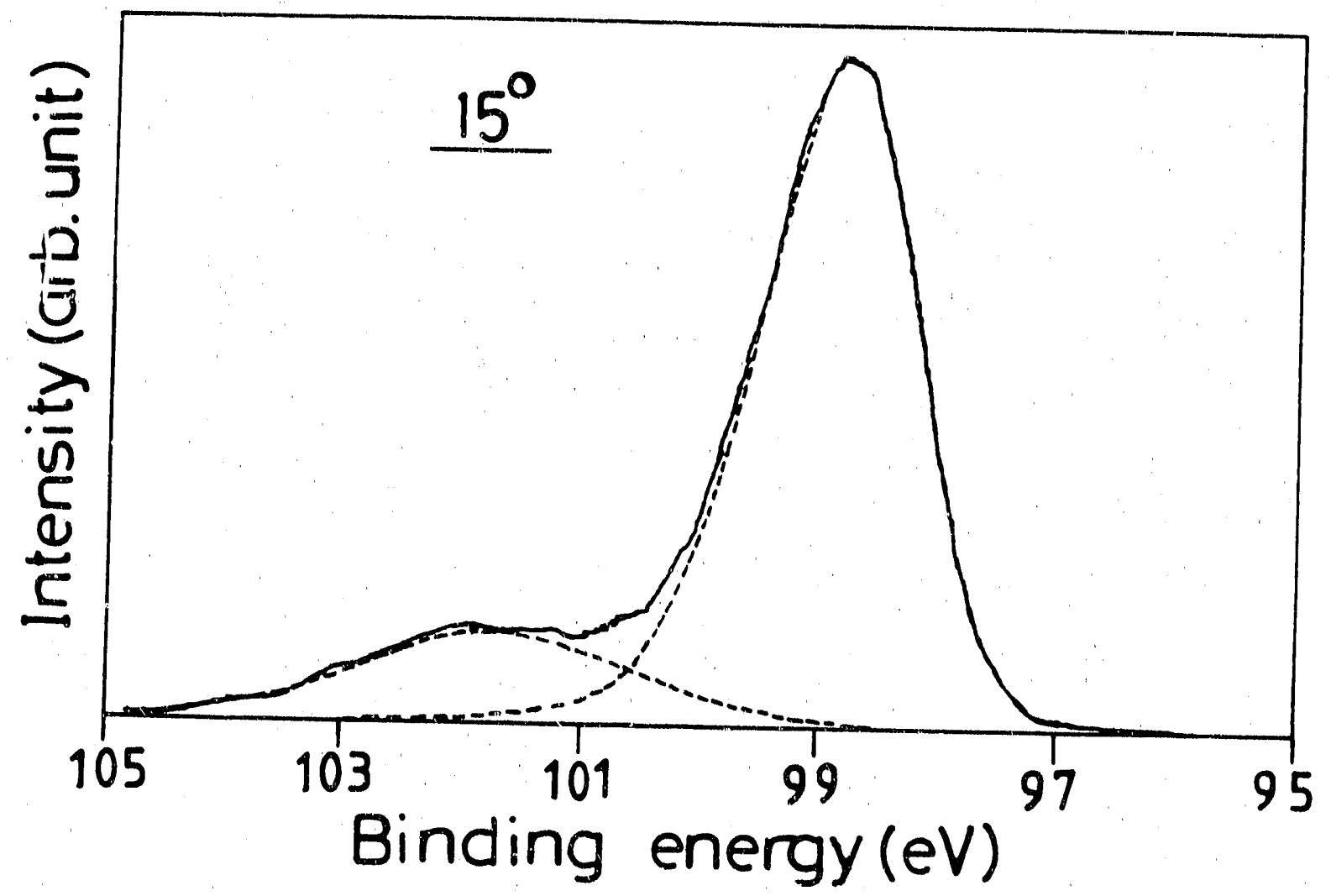

XBL $906-2132$

Figure 6.6: A Si 2p spectrum from a soft a-C:H film on silicon at electron takeoff angle of $15^{\circ}$. Deposition time is $20 \mathrm{~s}$. A suboxide of silicon is present at the interface of these films at submonolayer levels. 
proved to correlate well with the expected penetration depth of carbon ions into a silicon target, as determined by their kinetic energy.

The variation of the XPS signal with rf power is shown in Fig. 6.7 and Fig. 6.8. Fig. 6.7 shows the background subtracted Si 2 p spectra taken at an electron takeoff angle of $30^{\circ}$, for samples prepared at the powered electrode with uf power ranging from 10 to $100 \mathrm{~W}$. The control spectrum, shown in the upper left in Fig. 6.7, was taken of a sample which had received identical treatment as the other samples, excluding plasma ignition. It has been shown earlier, that for an $\mathrm{rf}$ power of $50 \mathrm{~W}$, the interface formation is complete well within 10 seconds of plasma ignition time and is simply being covered up by a. $\mathrm{C}: \mathrm{H}$ thereafter. Based on this result and the measured deposition rates, the deposition times for the other rf power levels shown in Fig. 6.7 have been chosen to be equivalent to or longer than the 10 second deposition time at $50 \mathrm{~W}$.

Using the same control parameters as noted above, the $\mathrm{Si} 2 \mathrm{p}$ spectra shown in Fig. 6.7, were separated into two overlapping peaks(dotted lines). The low binding energy peak originates from the pure silicon in the substrate, and the second peak about $1.8 \mathrm{eV}$ higher in binding energy is assigned to the silicon carbide[4] at the interface. It can be seen in Fig. 6.7, that the intensity of the interfacial peak relative to the peak associated with the substrate increases monotonically with $\mathrm{rf}$ power. As will be verified later, this change in relative intensity is due to a net increase in the thickness of the carbide interface with rf power, or more precisely, with the self bias voltage at the powered electrode.

The effect of increasing interface thickness with self bias voltage can also be seen in the $\mathrm{C}$ 1s spectra shown in Fig. 6.8. This figure shows the $\mathrm{C}$ 1s spectra taken at an electron takeoff angle of $60^{\circ}$ for the same series of rf power. The most intense contribution to the total signal is clearly that originating from the $\mathrm{a}-\mathrm{C}: \mathrm{H}$ overlayer. In this case, the control spectrum, shown in the upper left, is that taken of a sample covered by a sufficiently thick a-C:H layer (ca. $50 \AA$ ), to suppress the signal originating from the interface region. The extended tail noticeable on the higher binding energy side of the main peak is likely due to carbon contaminants. The intensity of the $C$ s signal from the a-C:H overlayer is so much greater than that from the carbide interface that 

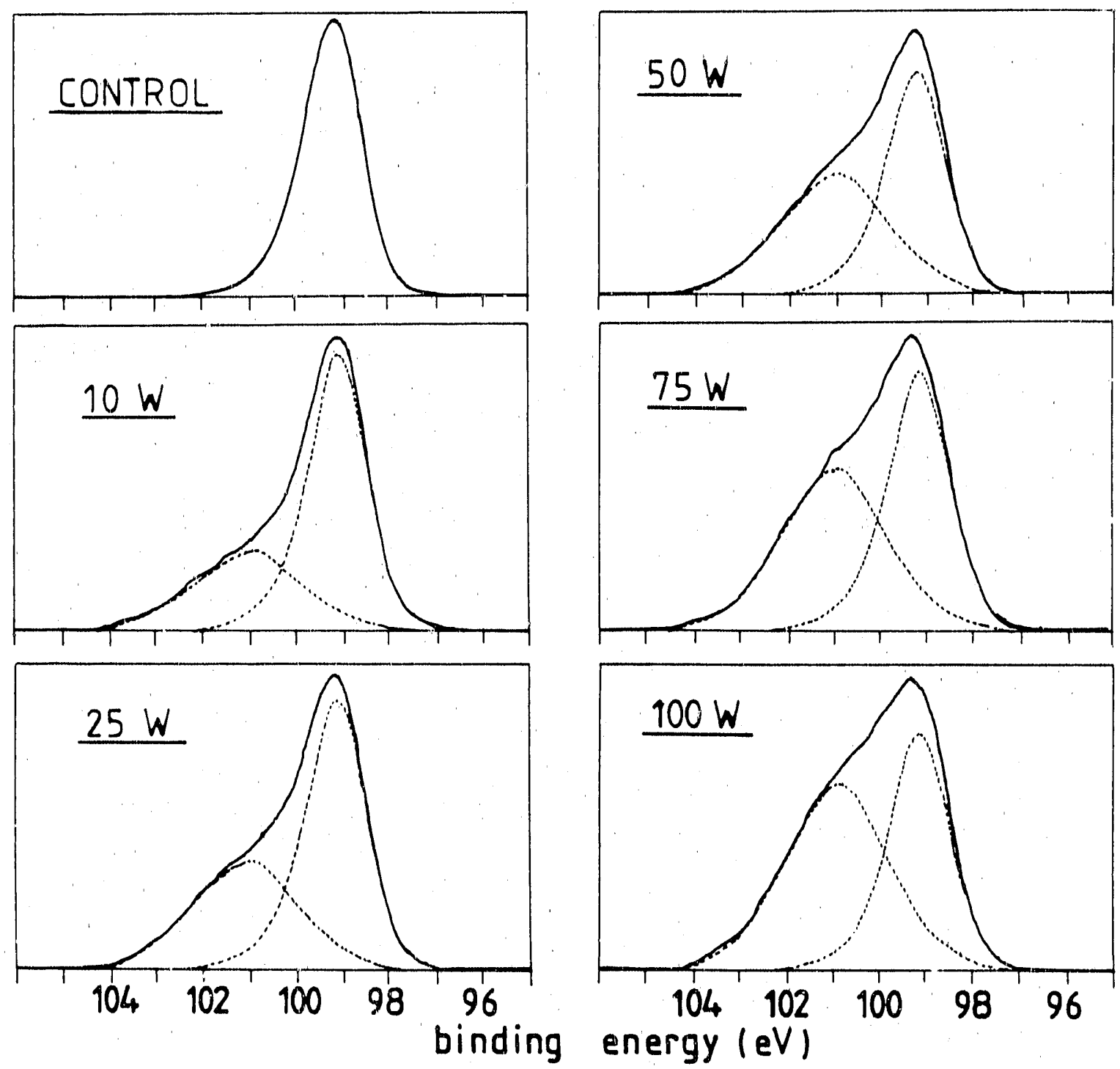

XBL $906-2131$

Figure 6.7: $\mathrm{Si} 2 \mathrm{p}$ spectra of a-C:H films as a function of self bias voltage. These spectra were taken at an electron take off angle of $30^{\circ}$, with if power ranging from 10 to $100 \mathrm{~W}$. 

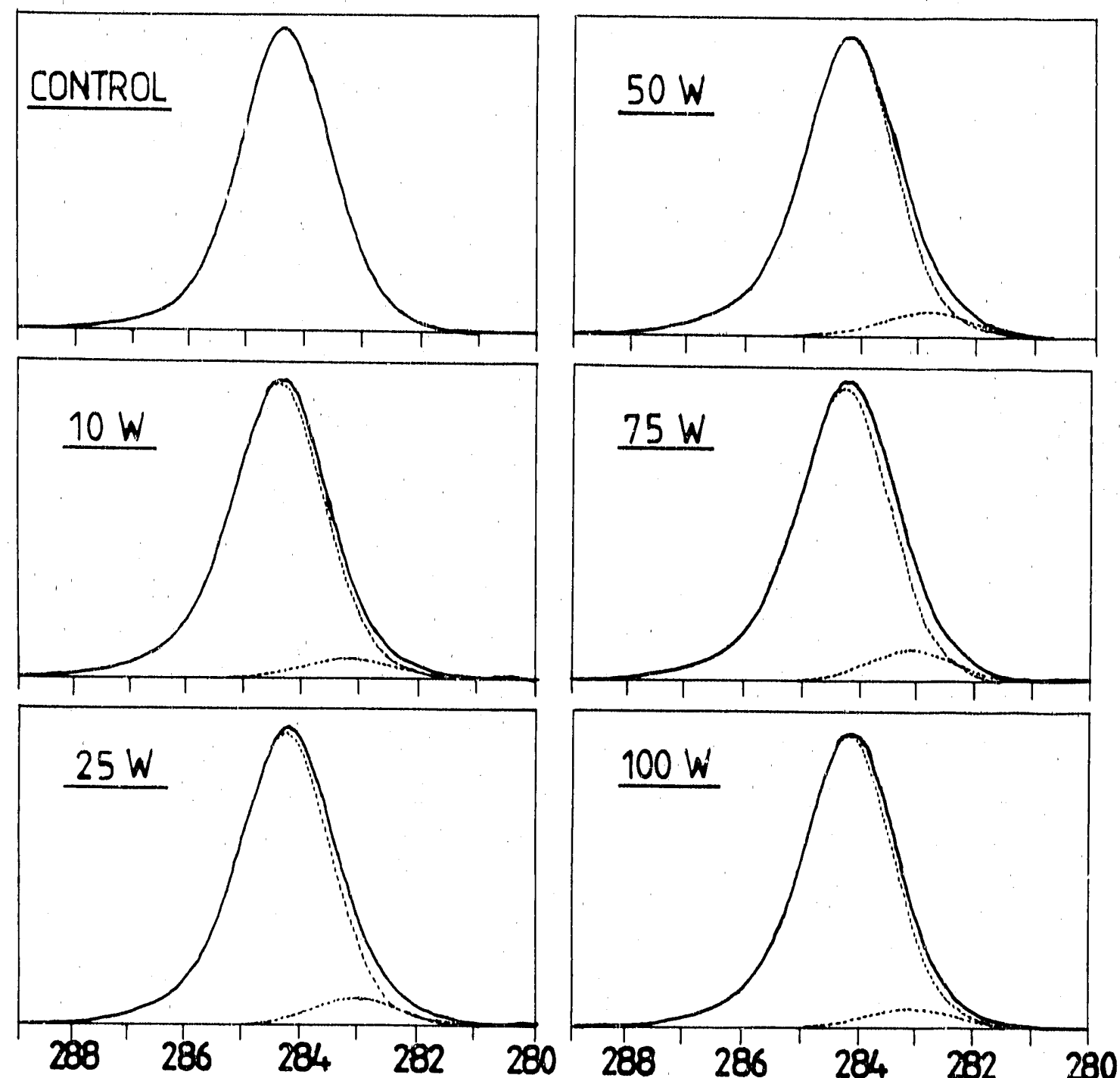
binding energy (eV)

XBL 906-2133

Figure 6.8: C 1s spectra of a-C:H films as a function of self bias voltage. These spectra were taken at a take off angle of $60^{\circ}$ for rf powers ranging from 10 to $100 \mathrm{~W}$. 
the contribution from the interfacial carbide is not inmediately apparent. However, after subtracting the intensity of the overlayer carbon, using the paramuters obtained from the control spectrum, a small peak at $283 \mathrm{eV}$, as expected for silicon carbide, was separable from the spectra. The intensity of these peaks gradually decreased with increasing deposition time, and also with decreasing electron takeoff angle, as expected for intensity originating from a buried interface.

As described earlier, information about the stoichiometry of the interfacial carbide can be obtained from the relationship between the interface related $\mathrm{Si} 2 \mathrm{p}$ and $\mathrm{C} 1 \mathrm{~s}$ peak areas. Fig. 6.9 shows the comparison of peak areas $\mathrm{fc}$ : the series of $\mathrm{rf}$ power values and electron takeoff angles. The scatter in the data is due mainly to error associated with the peak separation. The line drawn in Fig. 6.9 with a slope of unity shows a good average fit to the data, implying an approximately 1:1 ratio of silicon to carbon present in the interface. Fig. 6.9 thus confirms that, irrespective of rf power in the range form 10 to $100 \mathrm{~W}$, an approximately stoichiometric silicon carbide layer is formed at the interface of a-C:H films grown on silicon at the rf powered electrode.

Using Eq. 6.4, the thicknesses of the interface for films formed at various rf powers were calculated and the resuits are shown in Fig. 6.10 There is a good fit to the linear function of Eq. 6.4 for the data plotting $R(\theta)$ vs. $1 / \sin \theta$ for the series in rf power, with yome deviation at $\theta=15^{\circ}$. The fit to Eq. 6.4 is quite good considering that the assumption has been made that the materials are ideal uniform slabs, and parameters for single crystal silicon carbide were used for the interfacial carbide values $\rho_{i}$ and $\lambda_{i}$. Also noted in Fig. 6.10 is the interfacial thickness, $d_{i}$, determined from the slope of the line drawn for the series of rf powers. The thickness is approximately $4 \AA$ for $10 \mathrm{~W}$ and increases by about a factor of two at an rf power of $100 \mathrm{~W}$.

It is clear that the thickness of the interface increases with rf power. As the rf power is increased from 10 to $100 \mathrm{~W}$, the negative self bias voltage at the powered electrode changes from 150 to $540 \mathrm{~V}$. At the same time, however, the deposition rate also increases by a factor of five, which must be accompanied by a significant change in both the radical and ion flux to the substrate. It would be useful to determine which factor plays the most important role in controlling the thickness of the interfacial carbide. In order to address this question, Fig. 6.11 displays a plot of the thickness 


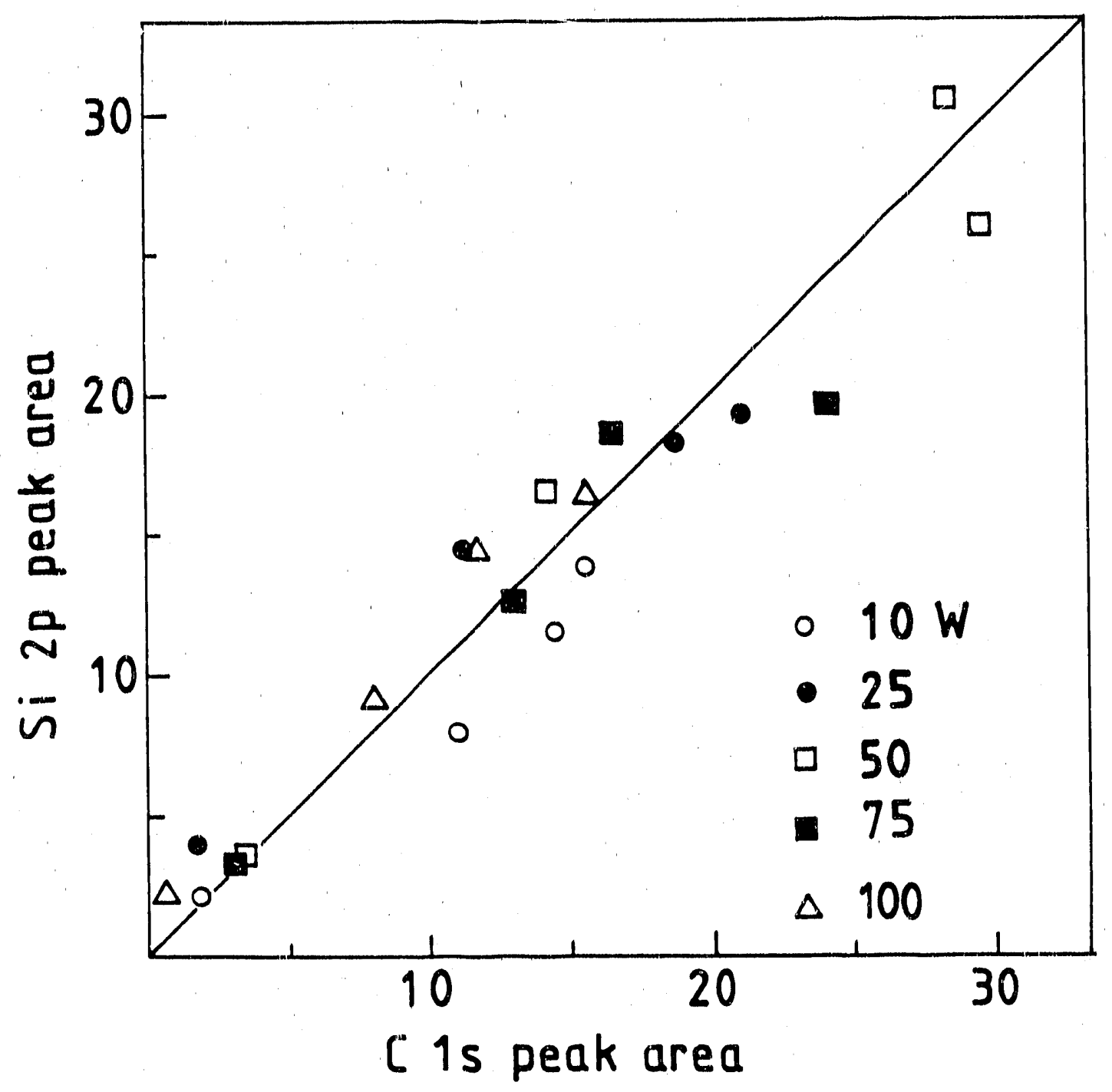

XBL $906-2124$

Figure 6.9: Relationship between the peak area of $\mathrm{Si} 2 \mathrm{p}$ and $\mathrm{C}$ 1s signals associated with the interfacial carbide. 


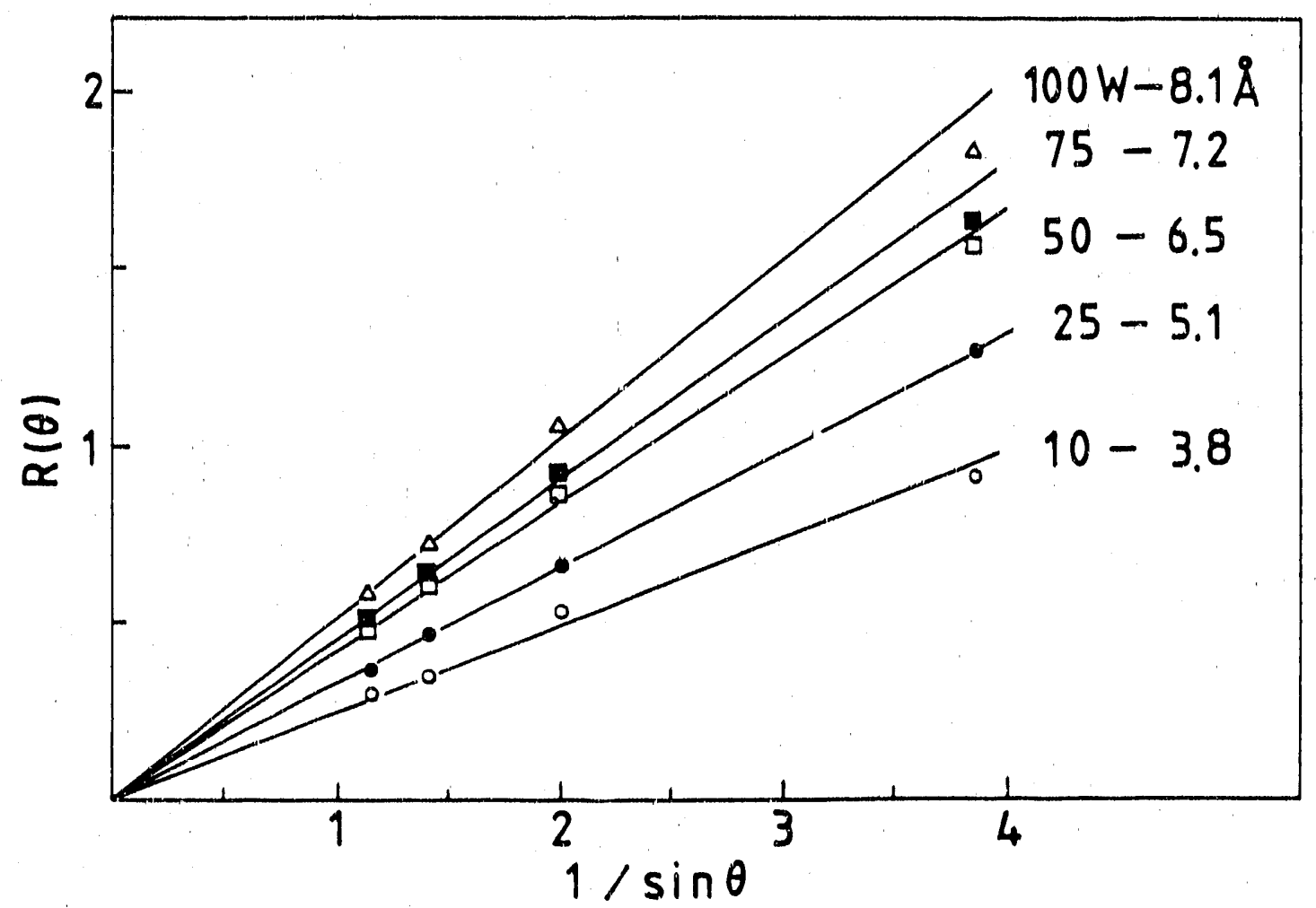

XBL 906-2123

Figure 6.10: Dependence of $R(\theta)$ as defined in the text on $1 / \sin \theta$, plotted for a series of self bias voltage conditions. The interface thickness, as calculated from the slope of the best fit lines are also given in the figure. 


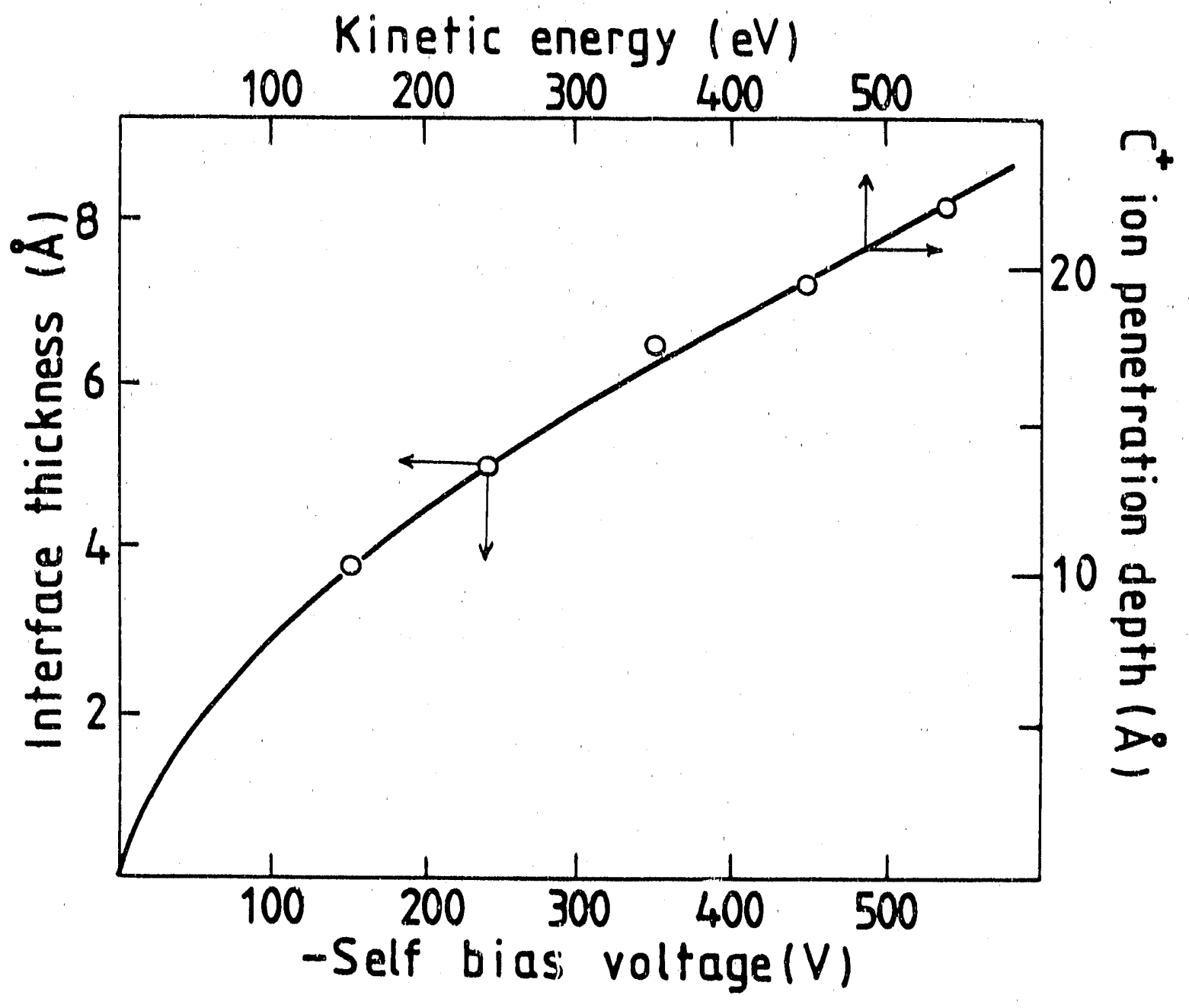

XBI $906-2122$

Figure 6.11: Calculated thickness of the silicon carbide interface as a function of self bias voltage. The solid line gives the results of the theoretical penetration depth of $\mathrm{C}^{+}$ions into silicon as a function of ion energy, as calculated using TRIM code. 
determined in Fig. 6.10, as a function of self bias voltage. Superimposed on this plot is a theoretical relationship between the average penetration depth of $\mathrm{C}^{+}$ions into a silicon target, as calculated by Brown[8] using TRIM code[7]. According to the measurement of the ion energy distribution in the plasma(see chapter 4, Fig. 4.7), the kinetic energy of plasma ions impinging on the substrate is essentially determined by the self bias voltage. Therefore, the upper and lower horizontal axes in Fig. 6.11 (kinetic energy and self bias voltage, respectively), are assumed to have the same scale. Fig. 6.11 shows that a vertical scale adjustment (the axis of average penetration depth is reduced by a factor of 2.7) results in a close fit of the kinetic energy dependence of the average penetration depth to that of the thickness of the silicon carbide interface.

In the plasma studied here, the dominant ions impinging on the substrate are not $\mathrm{C}^{+}$ions, but were found to be $\mathrm{CH}_{3}^{+}$ions (see Fig. 4.6). Theoretical calculations of the penetration depth of such bulky hydrogenated ions are difficult because their energy dissipation processes are complicated by bond breaking and resultant energy partitioning into the final fragments. It is reasonable, however, to expect that their penetration depth is significantly reduced, as compared to that of monoatomic $\mathrm{C}^{+}$ ions. This expectation does not necessarily justify a kinetic energy independent ratio of the penetration depth of $\mathrm{CH}_{3}^{+}$ions to that of $\mathrm{C}^{+}$ions. Nevertheless, Fig. 6.11 gives support to the notion that an intimate correlation exists between the thickness of the carbide interface and the penetration depth of plasma ions into the silicon substrate. The penetration depth is controlled by the kinetic energy of the incident ions, which in turn depends on the self bias voltage. It is therefore suggested that the thickness of the carbide interface is controlled by the self bias voltage, not by the ion and/or radical flux to the substrate.

\section{Pulsed Biasing Dependance of Interface Thickness}

The effect of pulsed biasing on interfacial thickness was also studied. Pulsed biasing serves as another useful method for controlling ion energy. Fig. 6.12 shows the Si $2 \mathrm{p}$ signal for the samples deposited under various pulsed biasing conditions, noting the pulse height and frequency. For comparison, Fig. 6.12 includes the spectra for films formed at the rf powered and the grounded electrode. As discussed earlier, 

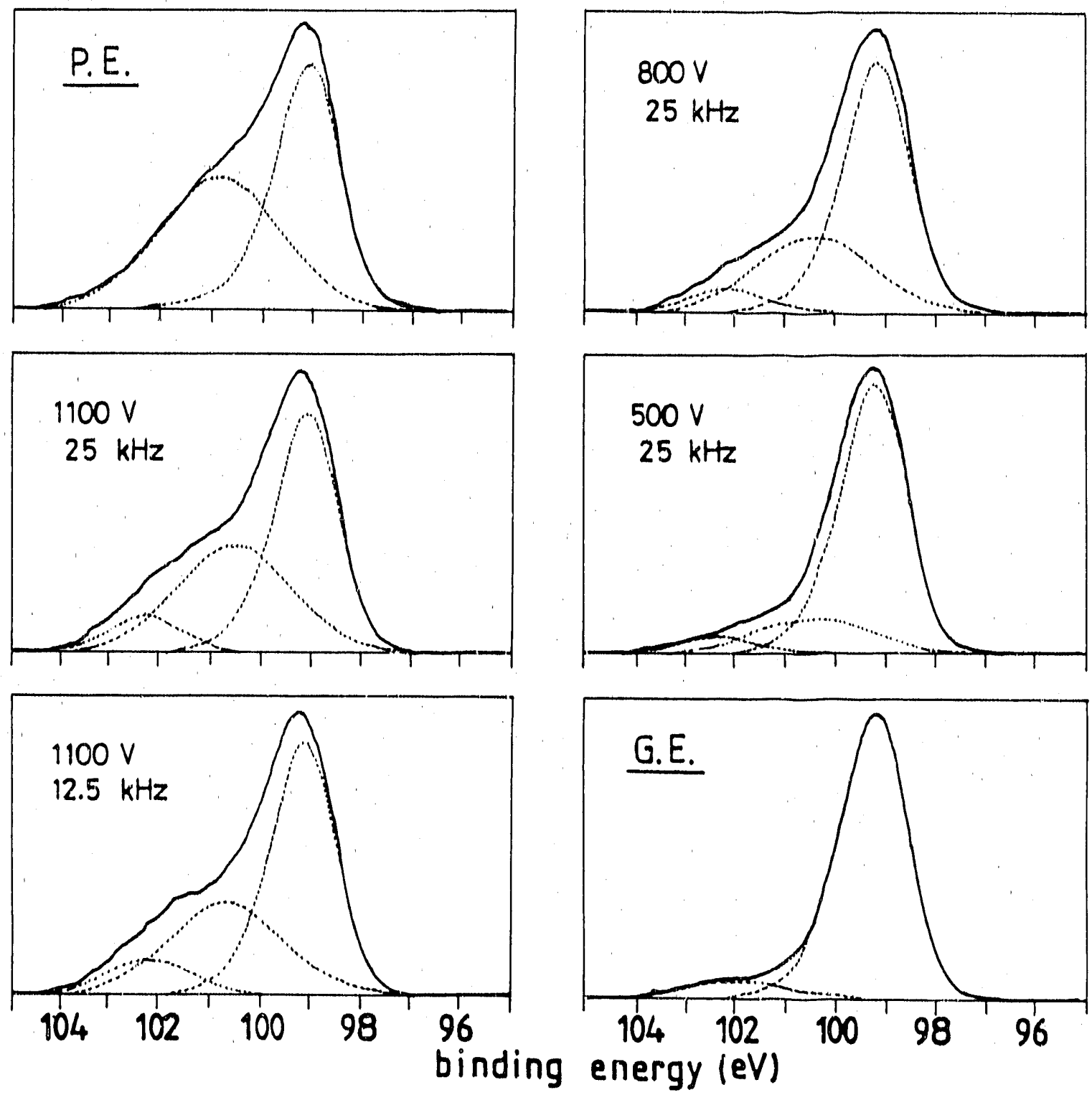

XBI 906-2129

Figure 6.12: Si 2p spectra for a-C:H films grown on silicon under various pulsed biasing conditions. For comparison, the spectra for films formed on the grounded and powered electrodes are included. 
no measurable silicon carbide interface exists in the case of the soft polymerlike film deposited at the grounded electrode. Instead, the grounded electrode Si $2 \mathrm{p}$ spectrum exhibitr intensity attributable to a small amount of oxide. Since the control Si $2 \mathrm{p}$ spectra with no a- $\mathrm{C}: \mathrm{H}$ deposition do not show any higher binding energy component, the oxide formation must be due to oxygen containing impurity radicals and/or ions in the methane plasma.

With the spectra for the powered electrode and grounded electrode as the two extremes, those obtained for films prepared at the pulsed biased electrode exhibit intermediate features. These spectra could not, however, be separated simply into two overlapping peaks from the substrate and the interfacial carbide, and a third component had to be included. The additional peak is at a binding energy corresponding to that of an oxide, and accounts for about $15 \%$ of the total higher binding energy signal in the Si $2 \mathrm{p}$ spectra. The rniddle peak which can be assigned to the interfacial carbide, also manifests a difference from that measured for carbide formed at the interface of films grown at the powered electrode, i.e. the chemical shift of the interfacial Si 2p signal is decreased. This is more clearly noticed as the pulsed bias voltage is decreased, and suggests that the interfacial carbide formed there has a silicon rich composition.

The peak separation of the $C$ is spectra also revealed small carbide related peaks at a binding energy similar to those in Fig. 6.2. A plot of the Si $2 p$ vs C 1 s peak areas, as shown in Fig. 6.13, offers additional evidence for the nonstoichiometric, silicon rich composition. The scatter of the data points is relatively large, but all the data falls in the regime of silicon rich composition.

With a pulse width of $2 \mu \mathrm{s}$, the time during which the substrate is subject to high energy ion bombardment is only about $5 \%$ of the total deposition time. If the high energy ions are responsible for the formation of the interfacial carbide, the silicon rich composition is not surprising under these deposition conditions. Here again, the average thickness of the nonstoichiometric silicon carbide layer is the quantity of major interest. The correlation which was noted between interface thickness and average ion penetration depth should be independent of stoichiometry.

It is obvious that some modification of Eq. 6.4 is necessary if it is to be applied 


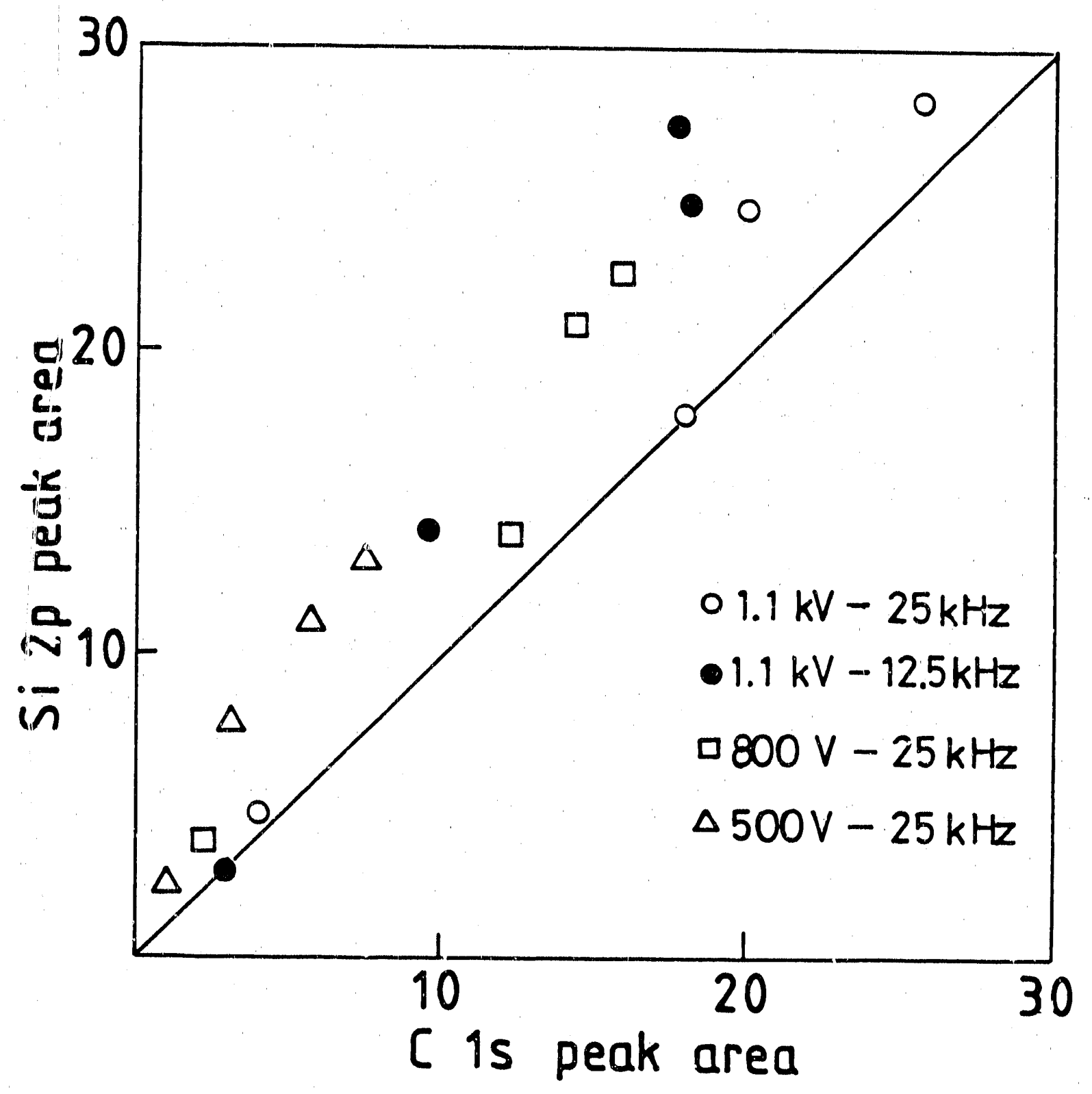

XBL $906-2116$

Figure 6.13: Relationship between the peak area of the Si 2p and C 1s signals associated with the interfacial silicon carbide layer. The scatter of the points is relatively large, but, all the data falls in the regime of silicon rich composition. 
to the silicon rich carbide layer. A plot of $R(\theta)$ as defined by Eq. 6.4 vs $1 / \sin \theta$ was found to be nonlinear. Here, two possible situations should be considered.

If the changes in the coordination of the silicon atoms in the interface could be ignored, parameters such as $\lambda_{i}$ and $\rho_{i}$ (electron mean free path and the atomic density of silicon in the interfacial layer) could simply be replaced by some average values between those of pure silicon and silicon carbide. This simple modification, however, did not lead to any improvement with respect to the linearity of the data.

More realistically, it must be taken into account that the average coordination of the silicon atoms in the carbide interface has been changed. The situation can be modeled most simply by assuming that two types of silicon exist in the interface; that coordinated as stoichiometric silicon carbide, and that coordinated as pure silicon. It follows that the pure silicon intensity in the XPS spectra does not originate only from the substrate, but some fraction thereof originates from the the interface region. This situation significantly aiters the angular dependence of the $\mathrm{Si} 2 \mathrm{p}$ spectra. Using this simple model to describe the corresponding angle dependence, $\phi$ is used to represent the volume fraction of pure silicon in the interface, and the interfacial parameters for the electron mean free path, $\lambda_{i}$, and the atomic density of silicon, $\phi_{\boldsymbol{i}}$, are given by:

$$
\begin{aligned}
& \lambda_{i}=\phi \lambda_{S i}+(1-\phi) \lambda_{S i C} \\
& \rho_{i}=\phi \rho_{S i}+(1-\phi) \rho_{S i C}
\end{aligned}
$$

The intensity of the Si $2 p$ signal at the normal binding energy is given by the sum of the intensities from the substrate and from the pure silicon atoms in the interface region:

$$
I_{s}(\theta)=I_{\theta} \lambda_{s} \rho_{s} \gamma_{c} \exp \left(-d_{i} / \lambda_{i} \sin \theta\right)+I_{\theta} \lambda_{i} \phi \rho_{S i} \gamma_{c}\left[1-\exp \left(-d_{i} / \lambda_{i} \sin \theta\right)\right]
$$

where $I_{\theta}$ accounts for the photoelectric emission cross section and the angle and energy dependent instrument factor, and $\gamma_{c}(<1)$ represents the coefficient of intensity attenuation due to the a-C:H overlayer. Note that $\rho_{s}=\rho_{S i}$. The intensity of the Si $2 \mathrm{p}$ signal due to the interfacial carbide is given by:

$$
I_{i}(\theta)=I_{\theta} \lambda_{i}(1-\phi) \rho_{S i C} \gamma_{c}\left[1-\exp \left(-d_{i} / \lambda_{i} \sin \theta\right)\right]
$$


The parameters $I_{\theta}$ and $\gamma_{c}$ cancel out of the equation when the intensity ratio, $I_{s}(\theta) / I_{i}(\theta)$, is calculated according to Eq. 6.7 and Eq. 6.8. By taking into account the relation of Eq. 6.5 and Eq. 6.6, transformation of this ratio results in a new formula of the following form:

$$
R^{\prime}(\theta)=\ln \left(1+\frac{\left[\lambda_{s} \rho_{s} / \lambda_{i}\left(\rho_{i}-\rho_{s} \phi\right)\right]}{\left[I_{s}(\theta) / I_{i}(\theta)-\phi \rho_{s} /\left(\rho_{i}-\rho_{s} \phi\right)\right]}\right)=\left(d_{i} / \lambda_{i}\right)(1 / \sin \theta)
$$

It can be easily verified that for $\phi=0$, Eq. 6.9 reduces to Eq. 6.4. In Eq. 6.9, the parameter $\phi$ can be varied to achieve the best linearity between $R^{\prime}(\theta)$ and $1 / \sin \theta$. The effect of varying $\phi$ on the linearity of the plot of $R^{\prime}(\theta)$ vs $1 / \sin \theta$ is shown in Fig. 6.14 for a typical sample produced using $-800 \mathrm{~V}$ pulsed biasing at $25 \mathrm{kHz}$. The best linearity was obtained with a pure silicon ratio of $\phi=0.49$, with which the interface thickness was calculated to be $11.9 \AA$. In this procedure, the presence of oxide intensity, as shown in the spectra of Fig. 6.12 could introduce error as Eq. 6.9 makes no allowance for the existence of an oxide. However, when the intensity of the oxide peak was formally included in $I_{i}(\theta)$, the optimum value of $\phi$ changed from 0.49 to 0.42 and the corresponding thickness from 11.9 to $11.3 \AA$. The error associated with the oxide, therefore, seems no greater than those errors associated with the other approximations involved in Eq. 6.9.

The above procedure was repeated for the other pulsed biasing conditions. It was found that the optimum pure silicon ratio varied from 0.34 for $1100 \mathrm{~V}(25 \mathrm{kHz})$ to 0.63 for $500 \mathrm{~V}(25 \mathrm{kHz})$. This is consistent with Fig. 6.12 and Fig. 6.13, which suggest that the interfacial carbide becomes more silicon rich as the pulsed bias voltage is decreased.

In Fig. 6.15, the thickness $d_{i}$, of the nonstoichiometric carbide interface is plotted as a function of the pulsed bias voltage. Carbide thickness is calculated from the slope of $R^{\prime}(\theta)$ vs $1 / \sin \theta$ using the optimum value $\phi$ for the pure silicon ratio. Over this data is again superimposed, the curve of average penetration depth of $\mathrm{C}^{+}$ions into silicon vs kinetic energy (scaled by a factor of 2.7), as was plotted in Fig. 6.11. The data for the nonstoichiometric interface display considerably more scatter than do the values for the stoichiometric carbide interface. This is likely due to the errors and approximations inherent in the complicated procedure for calculating the thickness of the nonstoichiometric silicon carbide interface. However, the agreement appears 

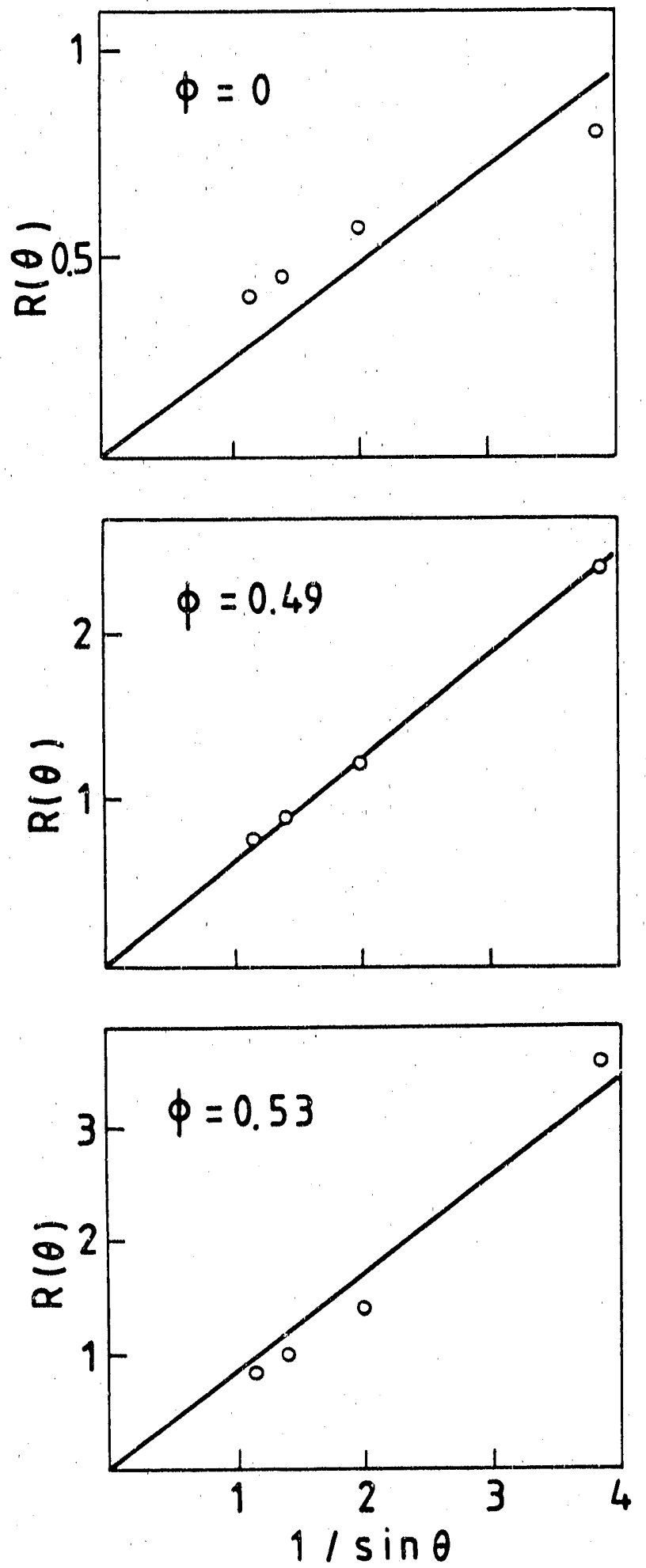

Figure 6.14: The variation in the linearity of the data as plotted as a function of the parameter $\phi$, which denotes the ratio of pure silicon co silicon carbide in the interface region, using the model described in the text. 


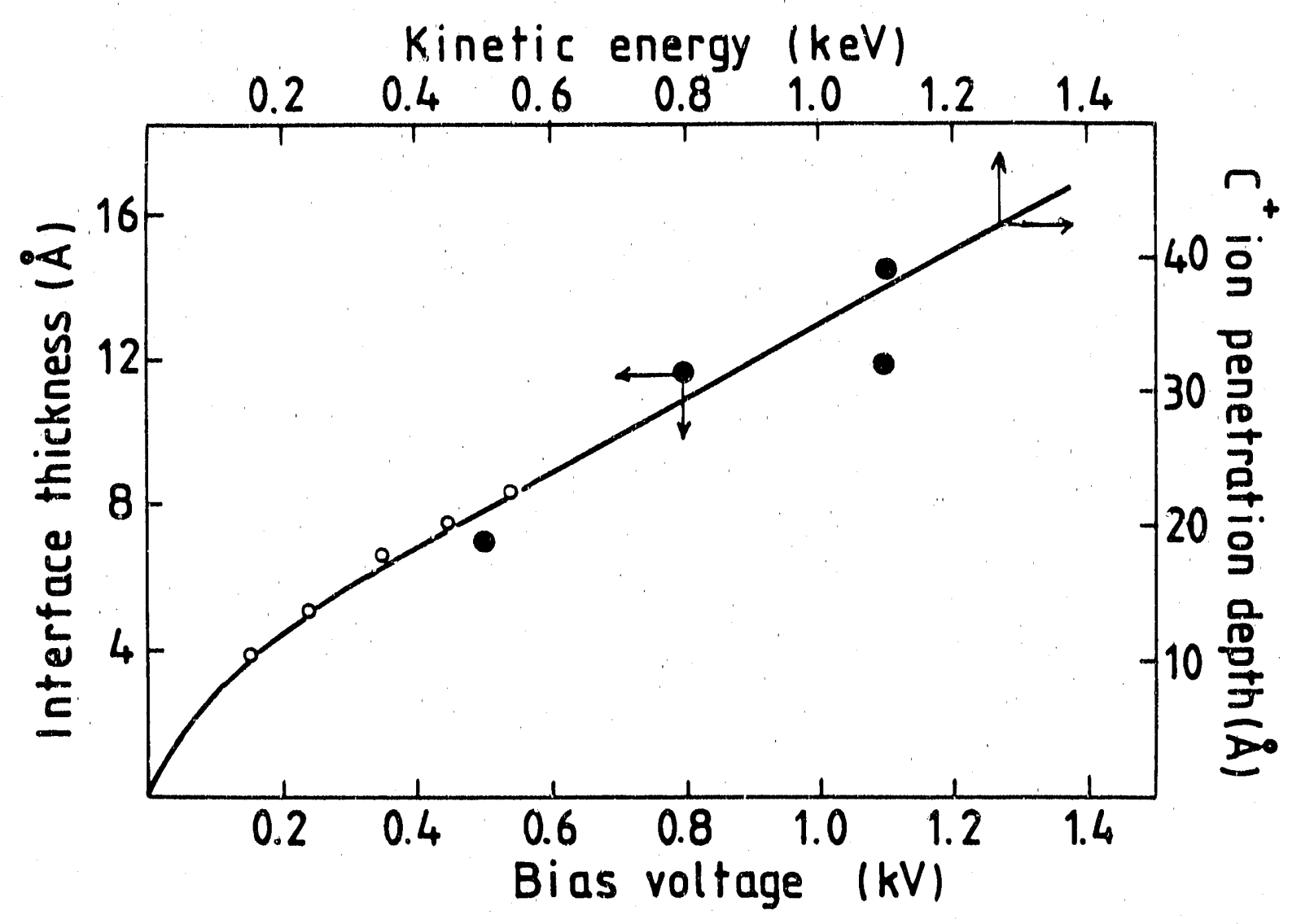

XBL $906-2121$

Figure 6.15: Calculated thickness of the silicon carbide interface as a function of pulsed biasing voltage. 
sufficient to support the noted correlation between the average penetration depth of the plasma ions and the thickness of the carbide interface. The correlation for these films appears irrespective of stoichiometry.

\subsection{SIMS}

In addition to the XPS study presented above, a series of SIMS depth profiling experiments were performed in hopes of extracting the interfacial composition. Both hard and soft a-C:H films more than $400 \AA$ thick on silicon were analyzed using 5 $\mathrm{kV}$ argon ion sputtering. The depth profiles as monitored with respect to $\mathrm{Si}_{n} \mathrm{C}_{m}^{+}$ions manifested an 'apparent' interfacial silicon carbide phase both for the hard and for the soft carbon films. The thickness ranged from 100 to $150 \AA$ in terms of FWHM of the interfacial peak in the depth profiles. The SIMS results are ircompatible with the interfacial thickness determined in the XPS study. It is apparent that ion depth profiling methods lack the depth resolution necessary for the study of thin interfacial layer, due to ion-induced atomic mixing with the substrate.

\subsection{Discussion}

Angle-resolved $x$-ray photoelectron spectroscopy has been used to investigate the interface of $\mathrm{a}-\mathrm{C}: \mathrm{H}$ films on silicon produced by plasma assisted chemical vapor deposition. The presence of approximately stoichiometric silicon carbide was demonstrated at the interface of hard a-C:H films produced under ion impact at the powered electrode. The effective thickness of the interfacial carbide was determined as approximately $7 \AA$ for films deposited using an rf power of $50 \mathrm{~W}$. The interfacial carbide layer is likely responsible for the strong adhesion of the hard a- $\mathrm{C}: \mathrm{H}$ films on silicon.

In contrast, no carbide layer was detected at the interface of soft polymer-like carbon films produced on silicon at the grounded electrode. The lack of the interfacial carbide could account for the poor adhesion of the polymer-like films on silicon.

The thickness of the interfacial carbide appears to correlate well with the average penetration depth of hydrocarbon ions in the plasma impinging on the substrate piaced on the powered electrode. Only those silicon atoms in the substrate which 
are accessible to the carbon containing ions from the plasma will finally be coordinated with carbon atoms to form the silicon carbide interface. In this sense, the intimate correlation between carbide thickness and average penetration depth seems reasonable.

Ion sputter depth profiling methods introduce mixing of the exposed surface layers and often do not have the necessary depth resolution for the study of thin layers. 


\section{Bibliography}

[1] G.J. Vandentop, M. Kawasaki, R.M. Nix, I.G. Brown, M. Salmeron, and G.A. Somorjai, Phys. Rev. B 41, 3220 (1990).

[2] P.Sander, U.Kaiser, M.Altebockwinkel', L.Wiedmann, L.Benninghoven, R.E.Sah, and P.Koidl, J. Vac. Sci. Technol. A, 5, 1470 (1987).

[3] M. Kawasaki, G.J. Vandentop, M. Salmeron, and G.A. Somorjai, Surface Science 227, 261 (1990).

[4] D.R. Wheeler and S.V. Pepper, Surf. Interface Anal., 10, 153 (1987).

[5] CRC Handbook of Materials Science, Volume III, (CRC Press Ohio 1975).

[6] R. Flitsch and S.I. Raider, J. Vac. Sci. Technol. 12, 305 (1975).

[7] J.F. Ziegler, J.P. Biersack, and U. Littmark, The Stopping and Range of Ions in Solids (Pergamon, New York 1985).

[8] I.G. Brown, private communication.

[9] G. Hollinger and F.J. Hirnpsel, J. Vac. Sci. Tech. A 1, 640 (1983). 


\section{Chapter 7}

\section{Nucleation and Growth by STM}

\section{$7.1 \quad$ Introduction}

As described in the previous chapter, the adhesive strength of a coating to its substrate is determined by the interfacial properties. The interface between a film and its substrate is formed during the initial stages of growth. Thus, a better understanding of the initia! stages of growth and of nucleation mechanisms is important if adhesion is to be controlled.

In this chapler, the results of an investigation of the initial stages of growth of aC:H films on graphite and silicon substrates by scanning tunneling microscopy (STM) and high resolution electron energy loss spectroscopy (HREELS) are presented. Interface formation mechanisms, involving plasma ions and free radicals, are discussed in light of the STM dava.

\section{2 a- $\mathrm{C}: \mathrm{H}$ on graphite}

Graphite substrates exposed to the methane plasma for deposition times between 5 and 20 seconds showed distinct cluster formation, as demonstrated by the $(1000 \AA)^{2}$ STM grey scale image in Fig. 7.1. This image was taken of a graphite substrate which had been exposed to the plasma for 15 seconds. The cluster diameter was seen to be typically about $50 \AA$. Cluster size remained roughly constant with deposition time while cluster density increased with deposition time. For deposition times greater than about 20 seconds, the clusters completely covered the graphite surface, leaving no exposed graphite where atomic resolution images of the graphite substrate could 


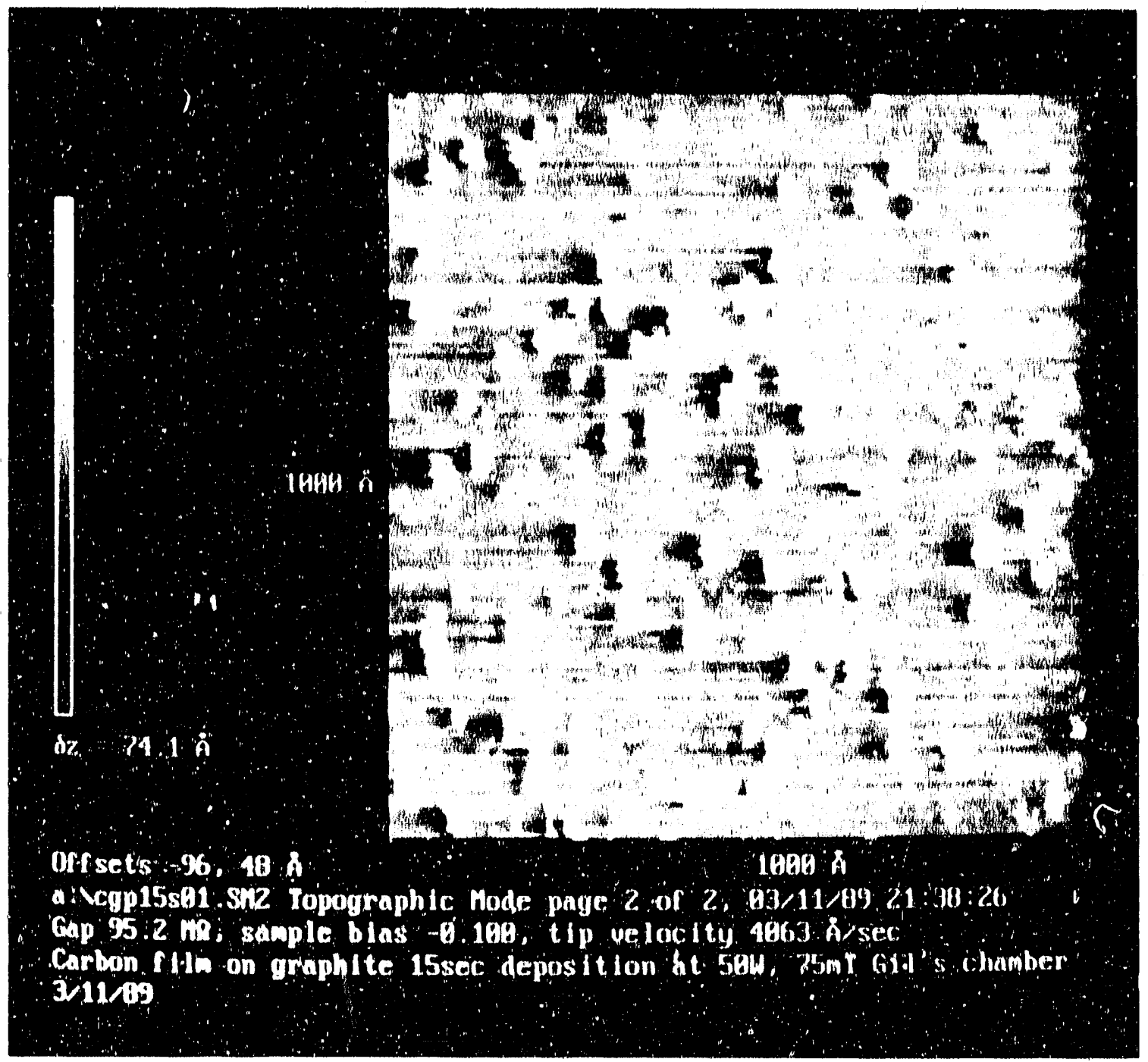

XBB 905-3946

Figure 7.1: 1000 by $1000(\AA)^{2}$ S'l'M lopographice image of a-C:All formation on graphite. 'This image is of an area of relatively low chuster donsity in the initial stages of growth. 
be obtained. An example of such a high covernge is shown in Fig. 7.2. Cluster deneity varied considerably from area to area on the same sample, as well as from sample to sample. Apparently, the plasme conditions were not spatially homogeneous during the intitial stagges of growth. The trend of incrensed cluster density as a function of time was clear, however, because of the noted lack of uniformity, it is not possible to quantify c'uster density behaviour as a function of time. Deposition times of 5 seconds always gave images showing a low clister density and thines greater than 25 seconds always showed complete coverage. However, STM images for samples with deposition times between 5 and 25 seconds, showed considerable fluctuation between the two extremes of cluster clensity. The electrically insulating properties of a-CH films with deposition times longer than 30 seconds made stable S'TM imaging impossible and these studies were not pursued.

The atomic structure of the individual clusters is of great interest. Marchon et al[1] succeeded in resolving atomic structure by STM at the surface of amorphous carbon films (non-hydrogenated) prepared by dc magnetron sputtering. These allthors observed small graphitic domains of approximately $15 \AA$ as well as numerous five membered rings. Other ordered domains which could be interpreted as being due to small diamond clusters were also observed. In these studies of the cluster structure, no diamond clusters were observed, and the most general appearance was that of disordered bright and dark spots. It is ciear that these clusters have an amorphous structure, lacking in any apparent local order even down to an atomic scale. Fig. 7.3 shows how the the regularly ordered atomic structure of the graphite substrate is observable between the a-C:H clusters, while the clusters themselves have an amorphous appcarance. Many of the closeup images of the clusters exhibited areas where four, five, and six membered rings appear to be present. Fig. 7.4 displays a closeup view of a cluster, demonstrating the amorphous structure. Some small areas of ringlike structures are also visible. These disordered structures, observed here for hydrogenated amorphous carbon, are reminiscent of the structures observed by Marchon for the case of nonhydrogenated carbon films.

The presence of five mombered rings in carbon films was predicted in 1973 by Nelson[2], as their bond angle of $108^{\circ}$ is close to the tetrahedral angle of $109.5^{\circ}$, 


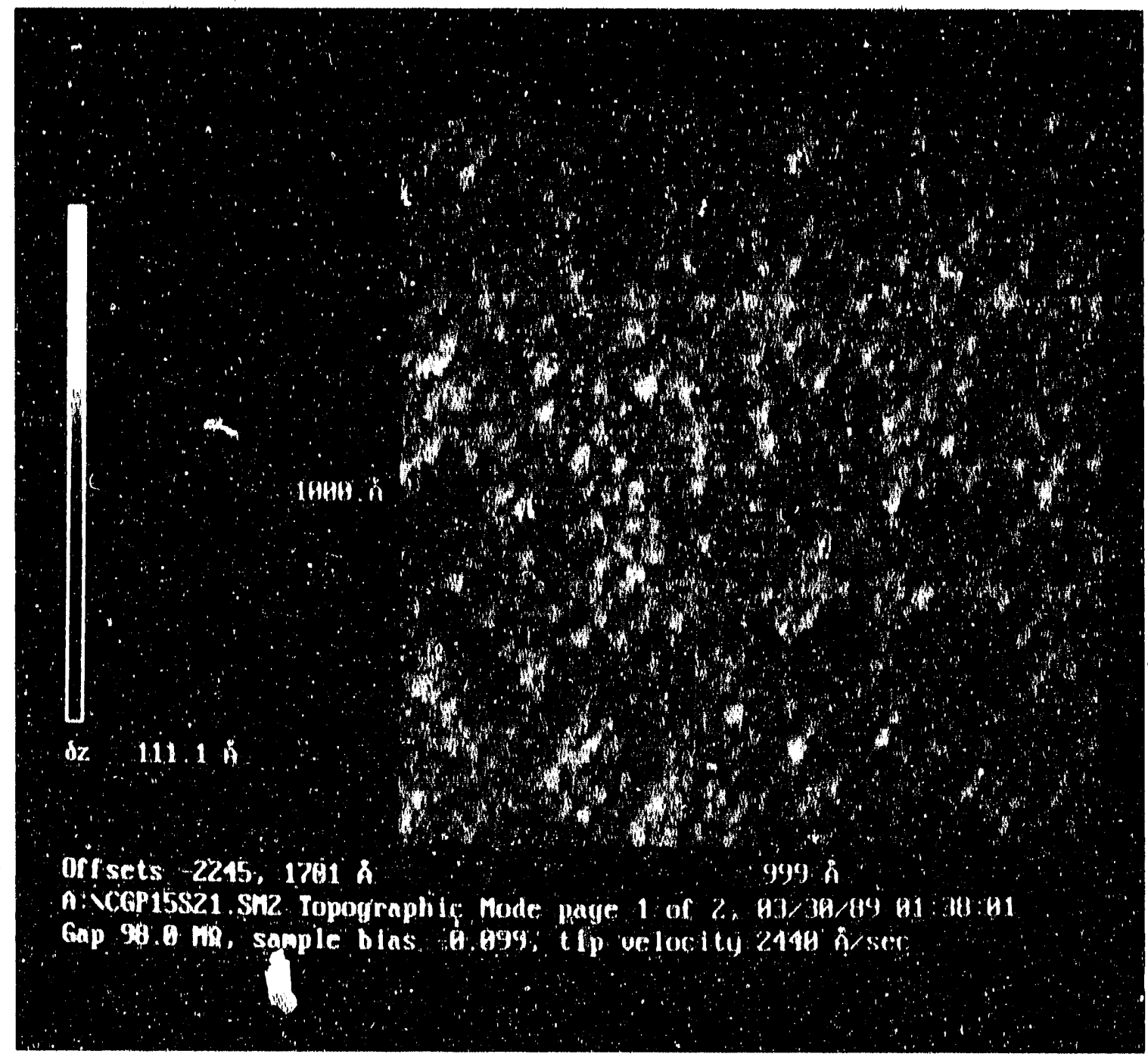

XBB 905-394T

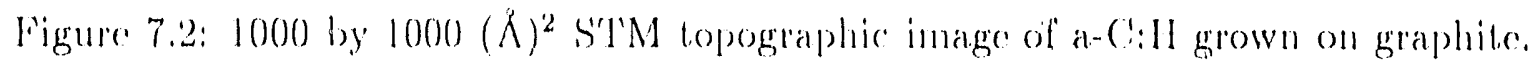
lligh cluster densily area. 


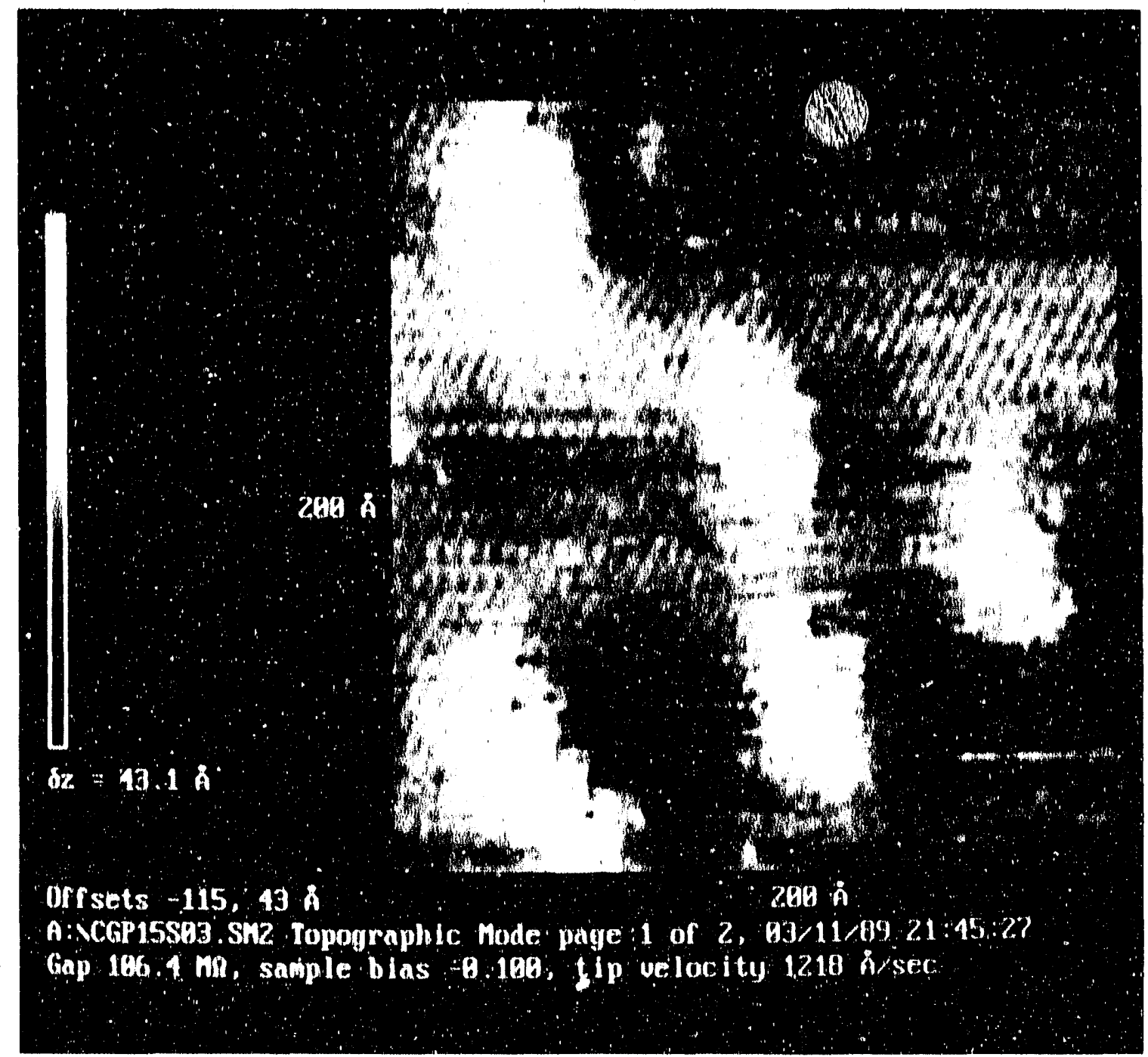

XBB $905-3950$

ligure $7.3: 200$ by $200(\AA)^{2} S^{\prime \prime} l^{\prime} \mathrm{M}$ topographic inage of chusters of a-C:H on a graphite surface. 'The regulan porionlicity' of the graphite substrate is visible in between the amocphons locking clustor's. 


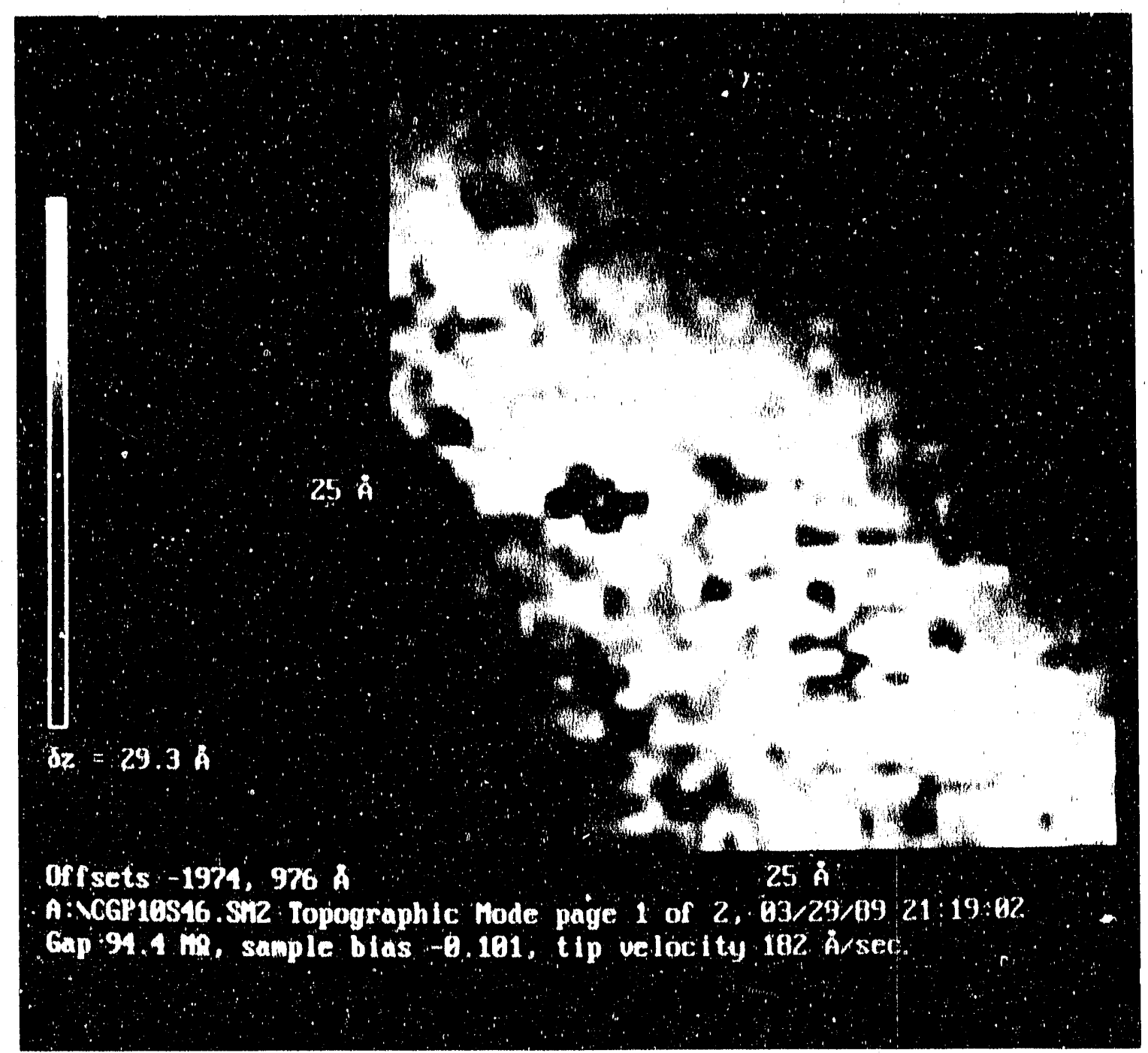

XBB 905-3951

Figure 7.4: 25 by $25(\AA)^{2}$ S'TiN topographic image of a cluster of a-C:H as formed on a graphite substrate. 
and their presence at the surface would result in fewer dangling bonds. In the case of hydrogenated carbon films; dangling bonds can of course be saturated with hydrogen, however the presence of nonhexagonal rings in a crosslinked carbon skeleton has been included in structures proposed in the past[3].

The presence of hydrogen at the surface of these films is dernonstrated in the HREELS spectra shown in Fig. 7.5. HREELS has been widely used as a surface vibrational technique in recent years, largely due to its high surface sensitivity $\left(10^{-2}\right.$ $10^{-3}$ of a monolayer) and its wide spectral range $\left(100\right.$ to $\left.5000 \mathrm{~cm}^{-1}\right)[4,5,6]$. Displayed in Fig. 7.5 are the HREELS spectra for an a-C:H film deposited for 10 seconds onto graphite at the self biased electrode, as well as that for a graphite blank substrate. The broad band at $1200-1400 \mathrm{~cm}^{-1}$ corresponds to $\mathrm{C}-\mathrm{H}$ bending vibrations, and the loss at $2935 \mathrm{~cm}^{-1}$ are associated with the $\mathrm{C}-\mathrm{H}$ stretch mode. In contrast, the graphite blank substrates wow no such modes. Thus, the presence of $\mathrm{C}-\mathrm{H}$ bonding is observed at the surface of the a-C:H films. As expected, this bonding is not present at the surface of the graphite blank, which received the identical treatment as did the a-C:H film, including exposure to the methane flow, and air transfer, but excluding plasma ignition. Similar features to those in the a-C:H spectra were observed for a semiconducting diamond (111) surface [7]. The width of the observed modes is quite large, even though the width of the elastic peaks $\left(75-90 \mathrm{~cm}^{-1}\right)$ is considerably smaller. This result is perhaps not surprising, considering the fact that the surface is both disordered and amorphous. Unfortunately, this fact prevents any unambiguous assignments of the bands observed to more specific vibrational modes. The presence of hydrogen at the surface of the a-C:H film, however, is clearly demonstrated, as is the absence of the same at the surface of the graphite blank.

It is interesting to compare the initial stages of growth in the case of films formed at the self biased electrode, where high energy ion bombardment takes place, to the growth of films formed at the grounded electrode, where this high energy bombardment is absent. Fig. 7.6 and Fig. 7.7. display this comparison. There is distinct cluster formation evident after the initial stages of growth at the self biased electrode, as shown in Fig. 7.6. The apparently random arrangement of clusters on the flat graphite substrate is clear in this three dimensional line drawing. In contrast, 


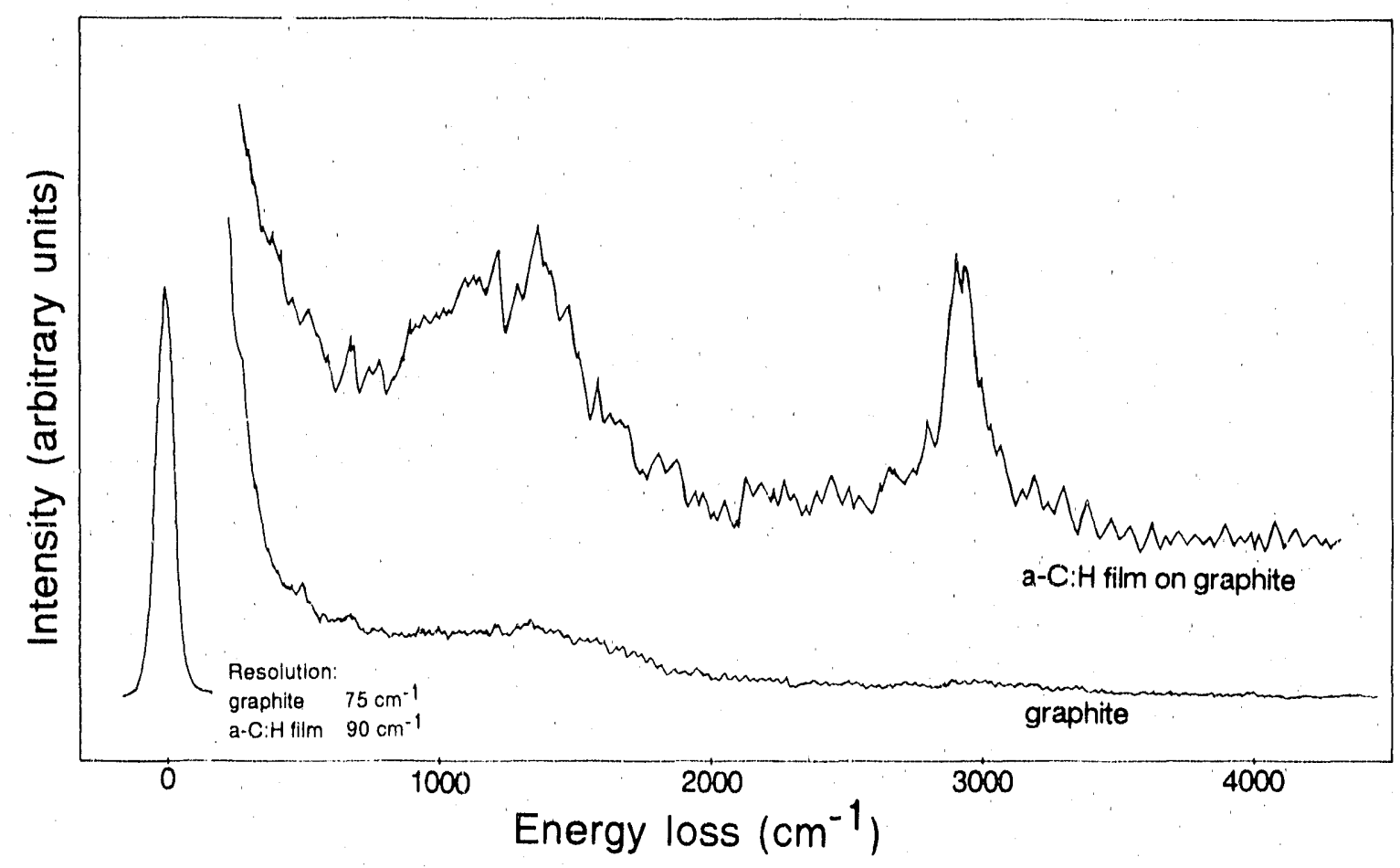

XBL $906-2137$

Figure 7.5: High resolution electron energy loss spectra of a graphite blank, and of a film of a- $\mathrm{C}: \mathrm{H}$ grown on graphite. The broad peaks at $1200-1400 \mathrm{~cm}^{-1}$ and around $2935 \mathrm{~cm}^{-1}$ correspond to $\mathrm{C}-\mathrm{H}$ bending and stretching vibrations, respectively. 


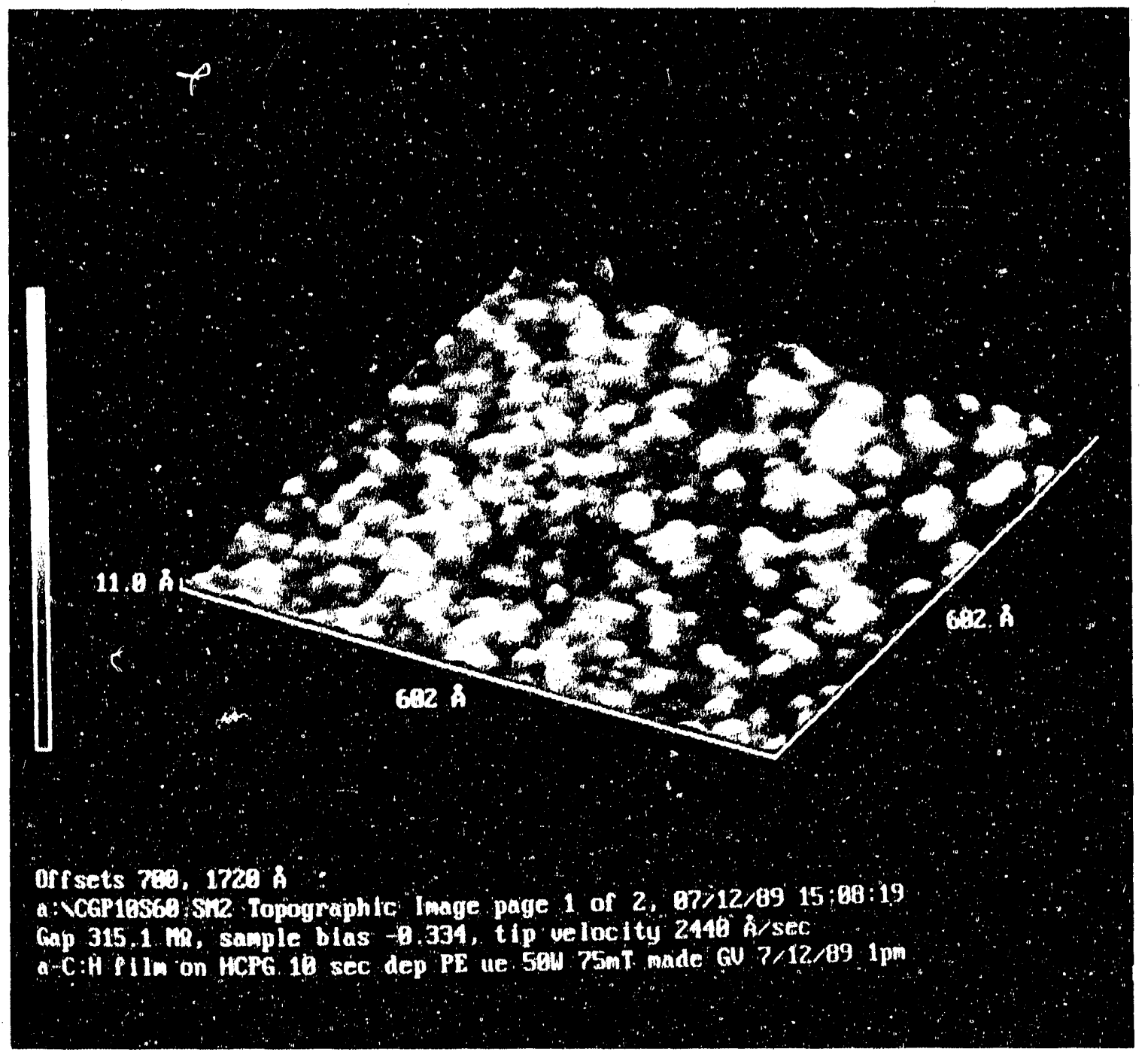

XBB $905-3949$

Figure 7.6: 'Three dimensional vicw of a topographic STM image taken of the initial stages of a (:H growth at the powered electrode. Cluster formation during the initial stages of growth is observable. 


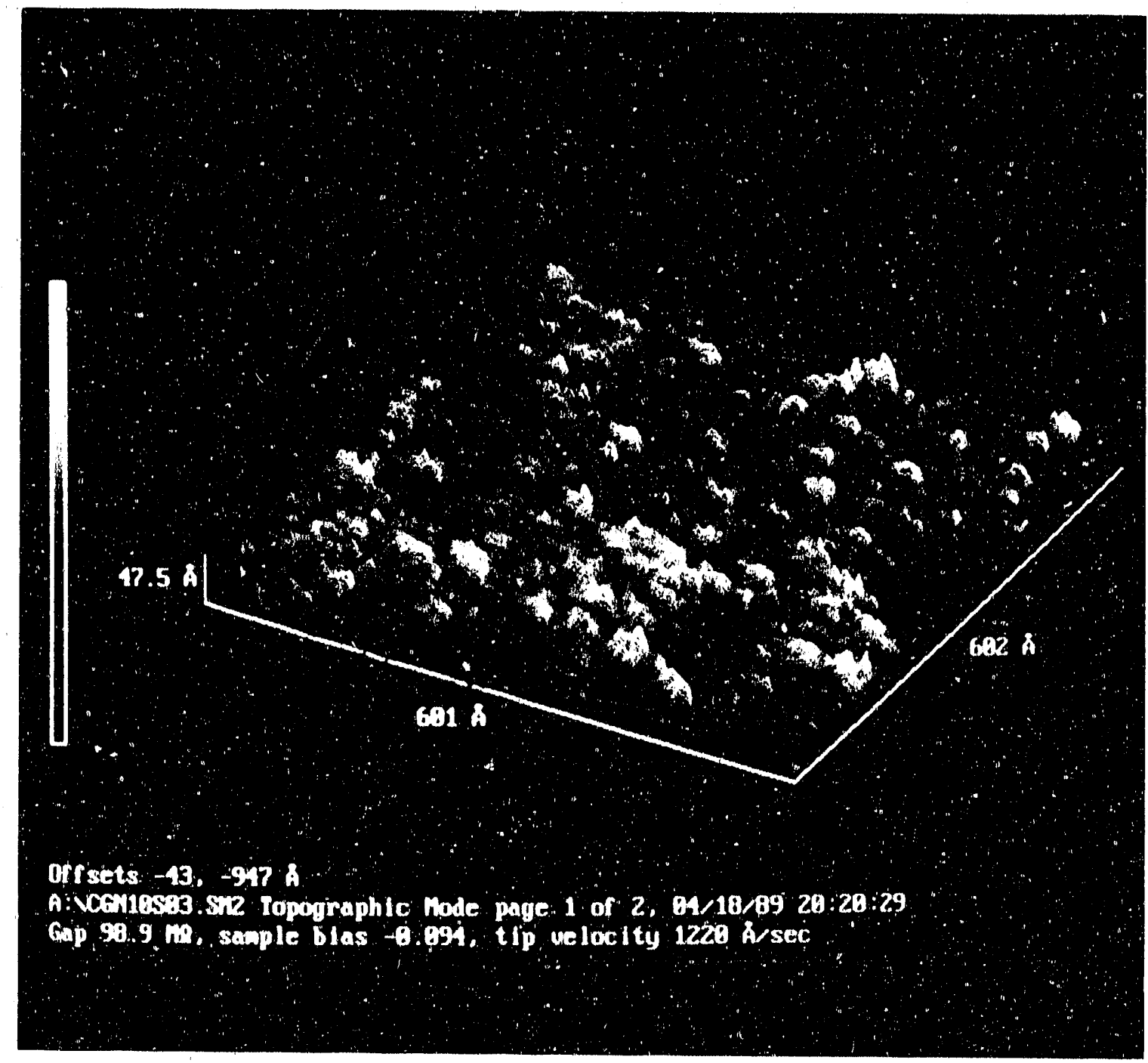

XBB 905-3954

Figure 7.7: Three dimensional view of topographic STM image talien of the initial stages of a- $(:$ Il growth at the grounded electrode. No cluster formation is observable. 
Fig. 7.7 shows an image of the film produced during the same deposition, but at the grounded electrode. In this case cluster formation is not observed.

\section{Novel Structures on Graphite}

On a number of occasions, a novel structure was imaged on the surface of graphite samples which had been exposed to the plasma. Fig. 7.8 displays a topographic STM image where $2 \times 2$ periodicity can be observed at various locations in the image. This sample was exposed to the methane plasma for 15 seconds at the powered electrode. Fig. 7.9, which is an inset of the image in Fig. 7.8, shows the double periodicity in the central and lower right area of the image. For comparison, the regular graphite periodicity is observable in an area in the lower left corner of Fig. 7.9. This double periodicity was also observed on graphite sample subjected to the methane plasma at the grounded electrode. Fig. 7.10 gives an example where the brightest spots are arranged in a $2 \times 2$ pattern, which is most prominent in the center of the image. At the top of the image, the regular periodicity of graphite is once again observable.

The origin of this double periodicity is not yet understood. It is not observable routinely on graphite samples which have been exposed to the plasma, but it has been measured on several occasions on a number of different samples and on a number of different days. As mentioned, it has been observed for graphite samples which have been exposed to the plasma both at the powered electrode and at the grounded electrode. It has never been observed on graphite blanks, not exposed to the plasma. To the best knowledge of the author, no such structure has been reported in the literature.

Possible explanations for these observations are numerous. The most attractive explanation is one attributing these structures as a periodic array of reactive plasma species which have adsorbed onto the graphite surface. It is also possible that chemical species have intercalated into the graphite around a defect which may or may have been created during the plasma exposure. Periodic arrangements of intercalates in graphite have been observed in the past by other techniques. The presence of chemical species arranged in this pattern is not a prerequisite for observation of such a pattern. A surface reconstruction could have occurred at the graphite surface, 


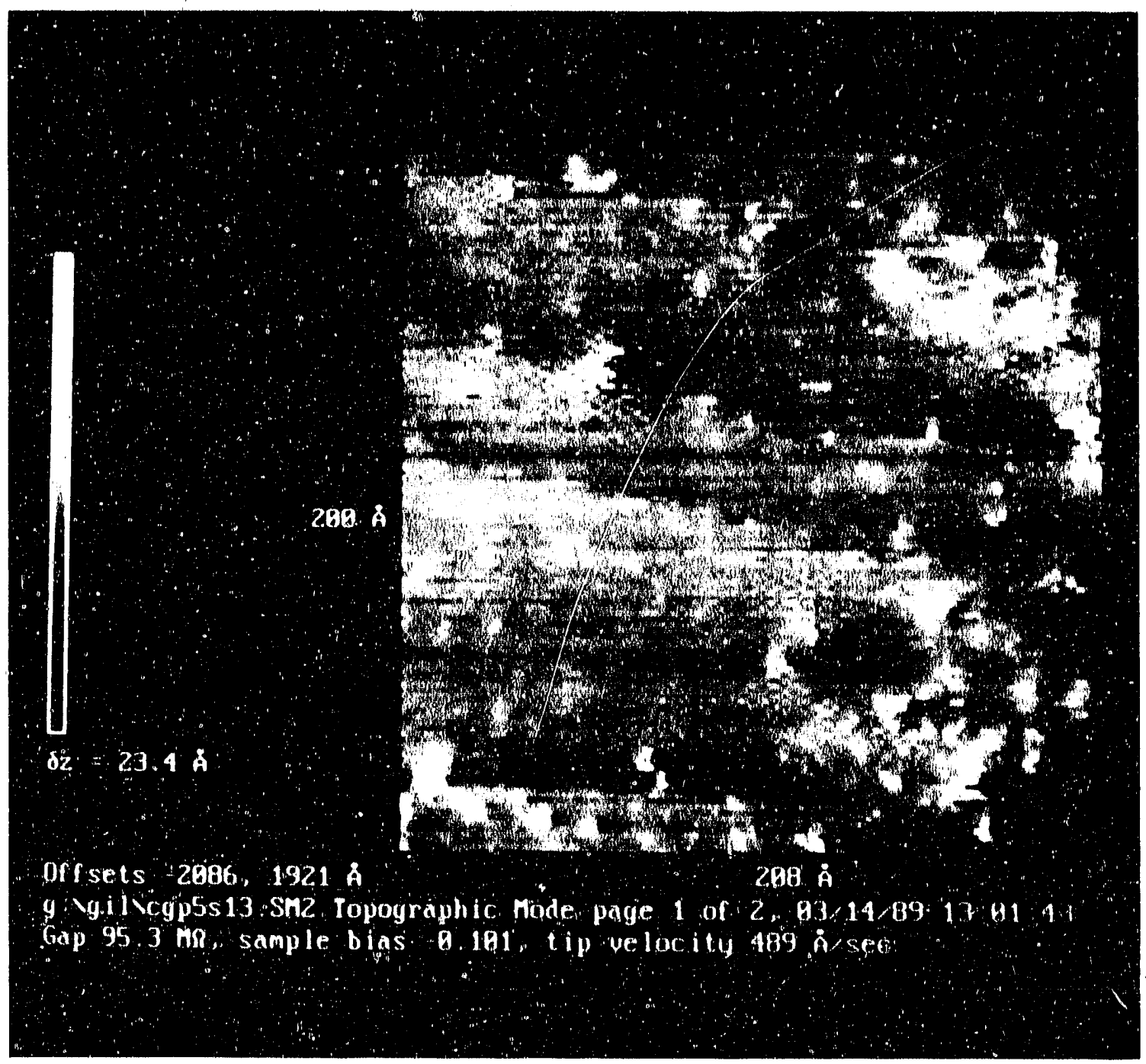

CBB 906-4722

Figure 7.8: 200 by $200(\AA)^{2}$ topographic s'l'M image displaying a number of areas where a $2 \times 2$ periodicity is observed on the surface of graphite which has been exposed to a methane of plasma. 


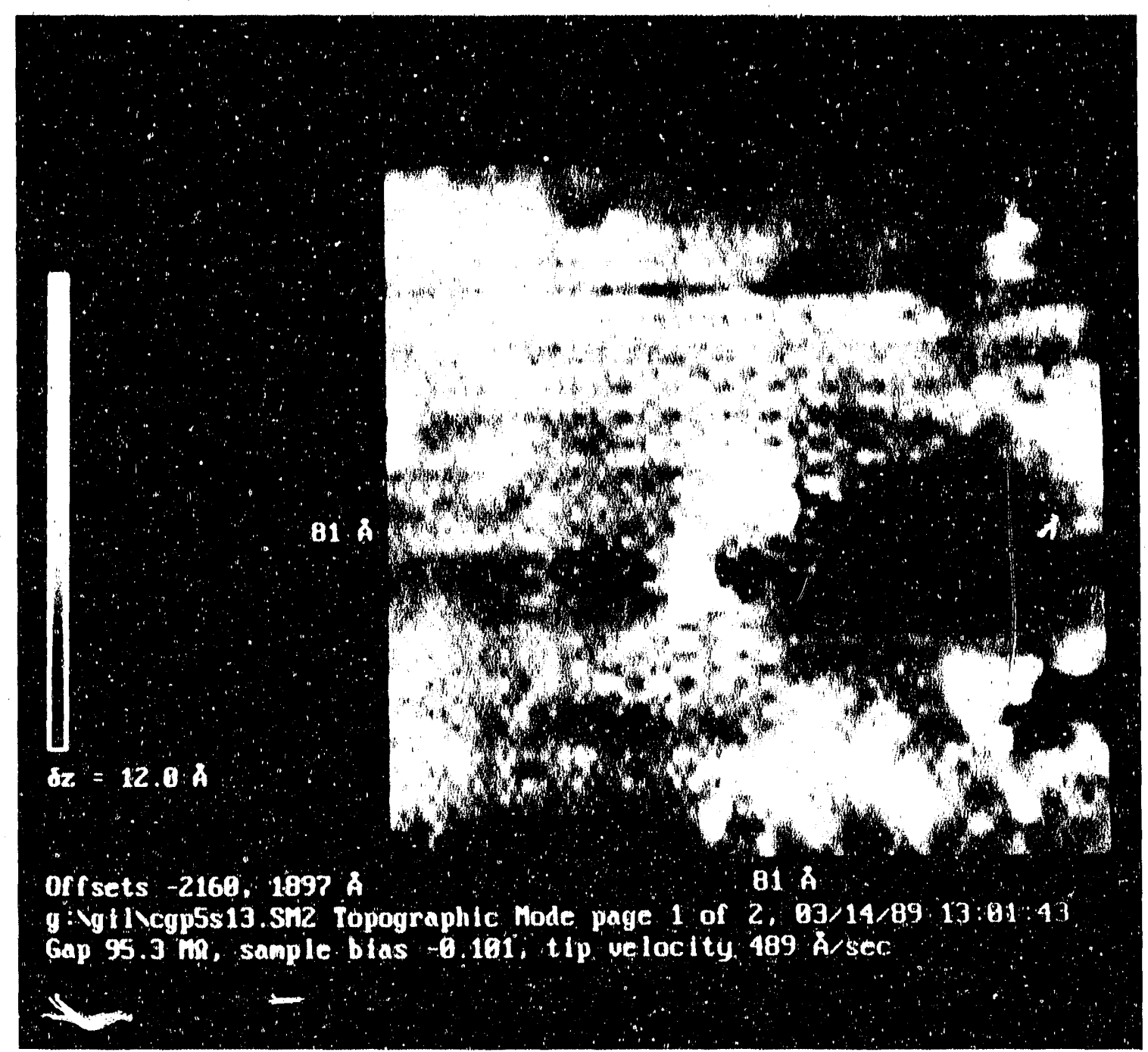

C.BB $906-4720$

Figure $7.9: 81$ by $81(\AA)^{2}$ inset of the previous figure. The double graphite periodicity is clear in the conter area of the image. In the lower left corner an area of regular graphite periodicity is observalible for comparison. 


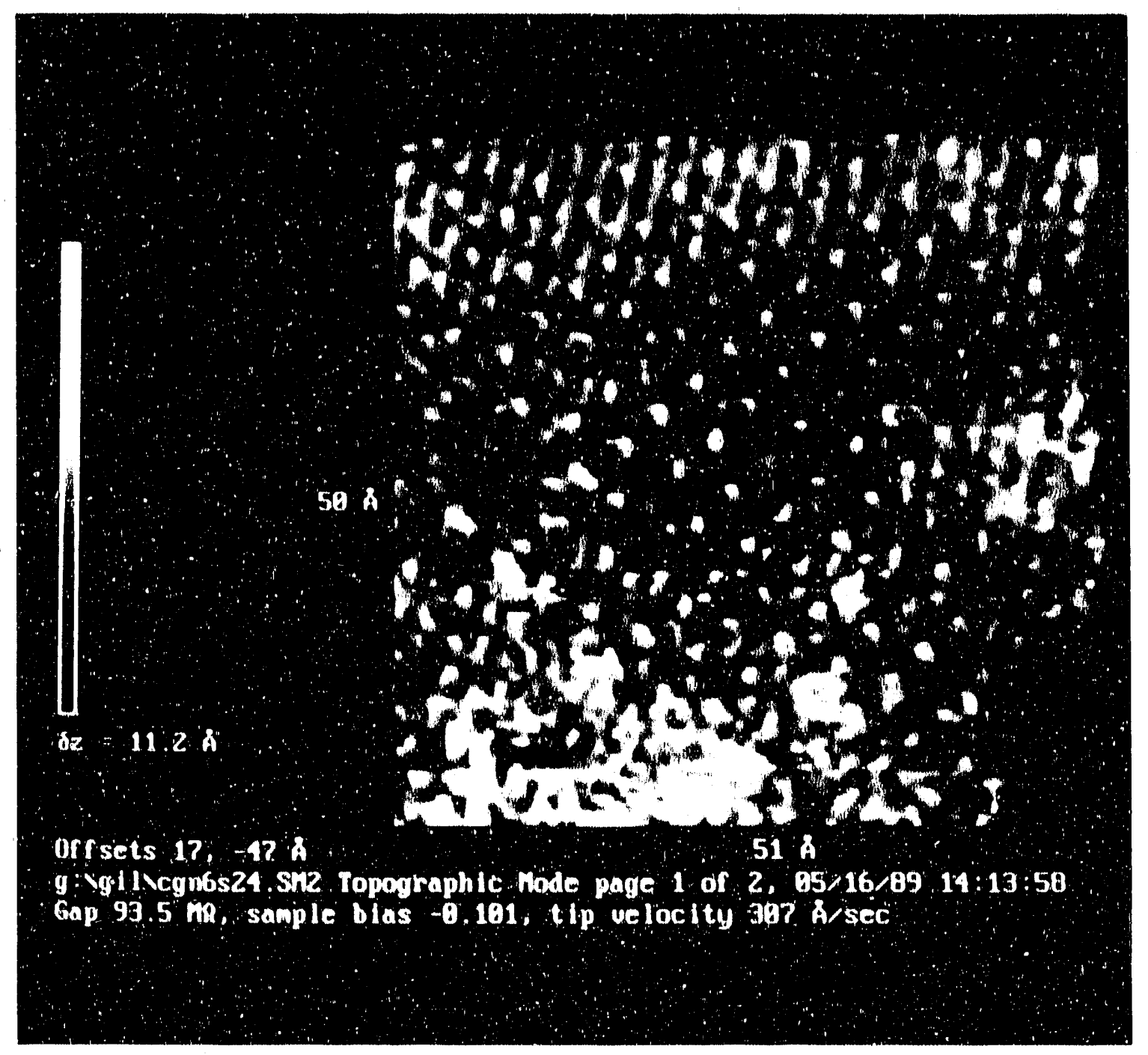

CBB 906-4724

Figure 7.10: 50 by $50(\AA)^{2}$ area of graphite exposed to a methane if plasma for 6 seconds at the grounded electrode. $A 2 \times 2$ periodicity in the structure is observable in the conter of the image towards the loft. 
possibly plasma initialed, which conld give rise to such a paltern. 'These explanations must, be treated as spoculation, since no additional evidence for these structures is available. Performing tumnelling electron spectroscopy[14] on these structures could lead to a better understanding of the electronic structure present, and could aid in an understanding. These structures are, unfortunately, intractable to diffraction techniques, such as low energy eloctron diffraction (LEED), as they are found only in small patches over the surface and when averaging over a significantly larger area, they would not be observol. One attempt at observing a $2 \times 2$ pattern on a plasma exposed graphite surface, by low energy electron diffraction, failed as expected.

\section{3 a-C:H on $\mathrm{Si}(100)$}

In order to facilitate the observation of features due to the initial stages of growth of a-C:H on silicon, it was first necessary to prepare a relatively featureless substrate. Using the techniques described in the experimental section silicon substrates were prepared with acceptable roughness. This is demonstrated in Fig. 7.11, where the image gave a standard deviation of tip positions around the mean of less than $3 \AA$. The silicon surface may have been even smoother than this, as the measured smoothness was limited by gap instabilities, and not on topographic structure. Substrate smoothness was stable for more than 24 hours.

The surface topography of a-C:H films grown on silicon substrates at the self biased clectrode (Fig. 7.12) was also exceedingly smooth. In fact the topography of the film surface was practically indistinguishable from the topography of the substrate surface ( $<3 \AA$ standard deviation from mean position). Some topographic features are barely visible in Fig. 7.12, but the height of these features is comparable to the noise level. It is clear that the clusters observed in the case of a-C:H formation on graphite are not observed in the case of silicon substrates. a-C:H films formed on silicon at the self biased electrode are flat to within $3 \AA$ for all deposition times from 5 to 20 seconds. Tunneling gap stability degraded with increasing thickness, as was the case on graphite substrates.

Unfortinately, no useful images were obtained for the case of a-C:II films formed on silicon at the grounded elctrode. The tunneling gap was not sufficiently stable 


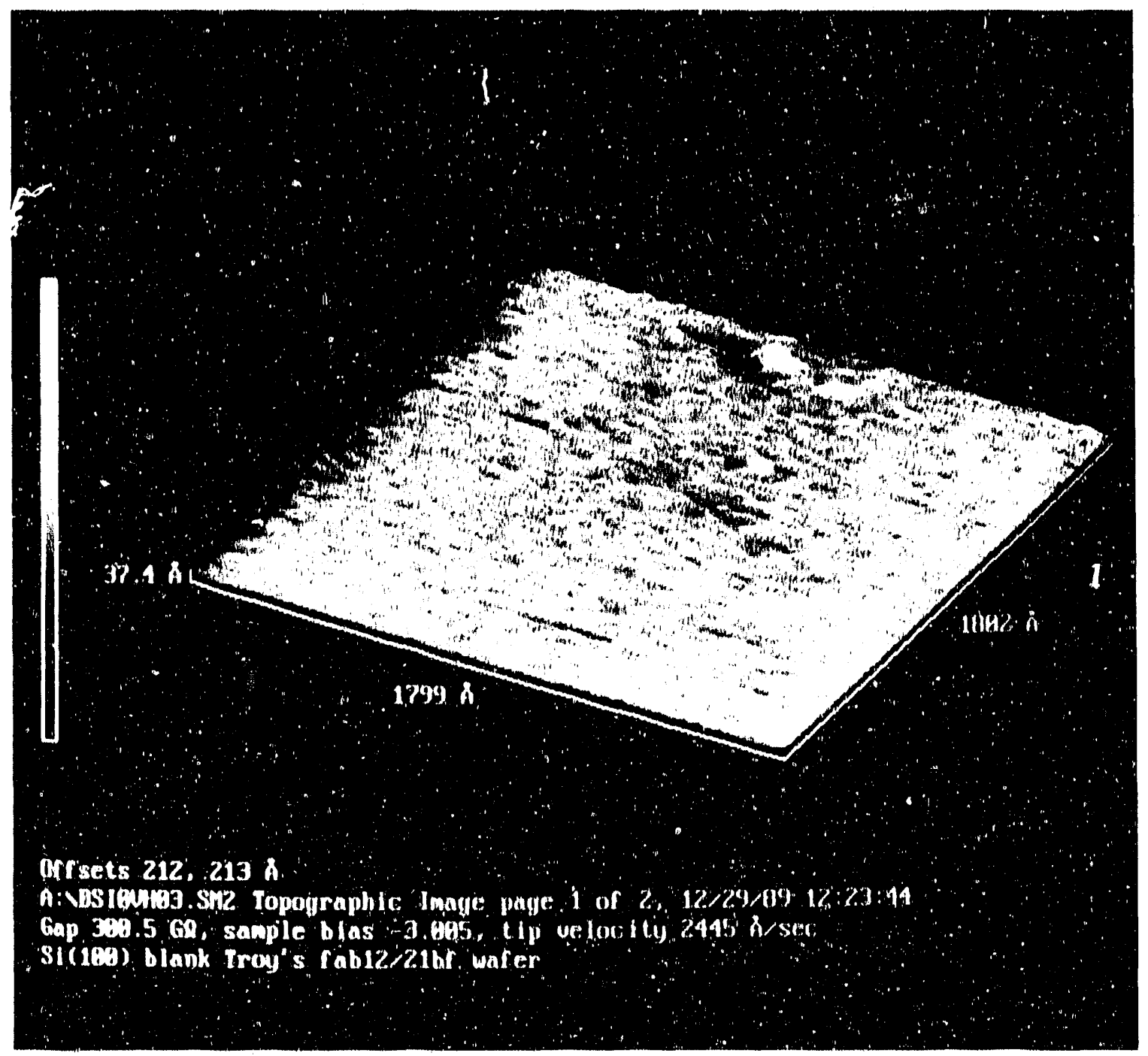

XBB 905-3948

Figure 7.11: 1800 by $1800(\AA)^{2}$ topographice S'T'M image of a lypical surface of a silicon blank substante. 


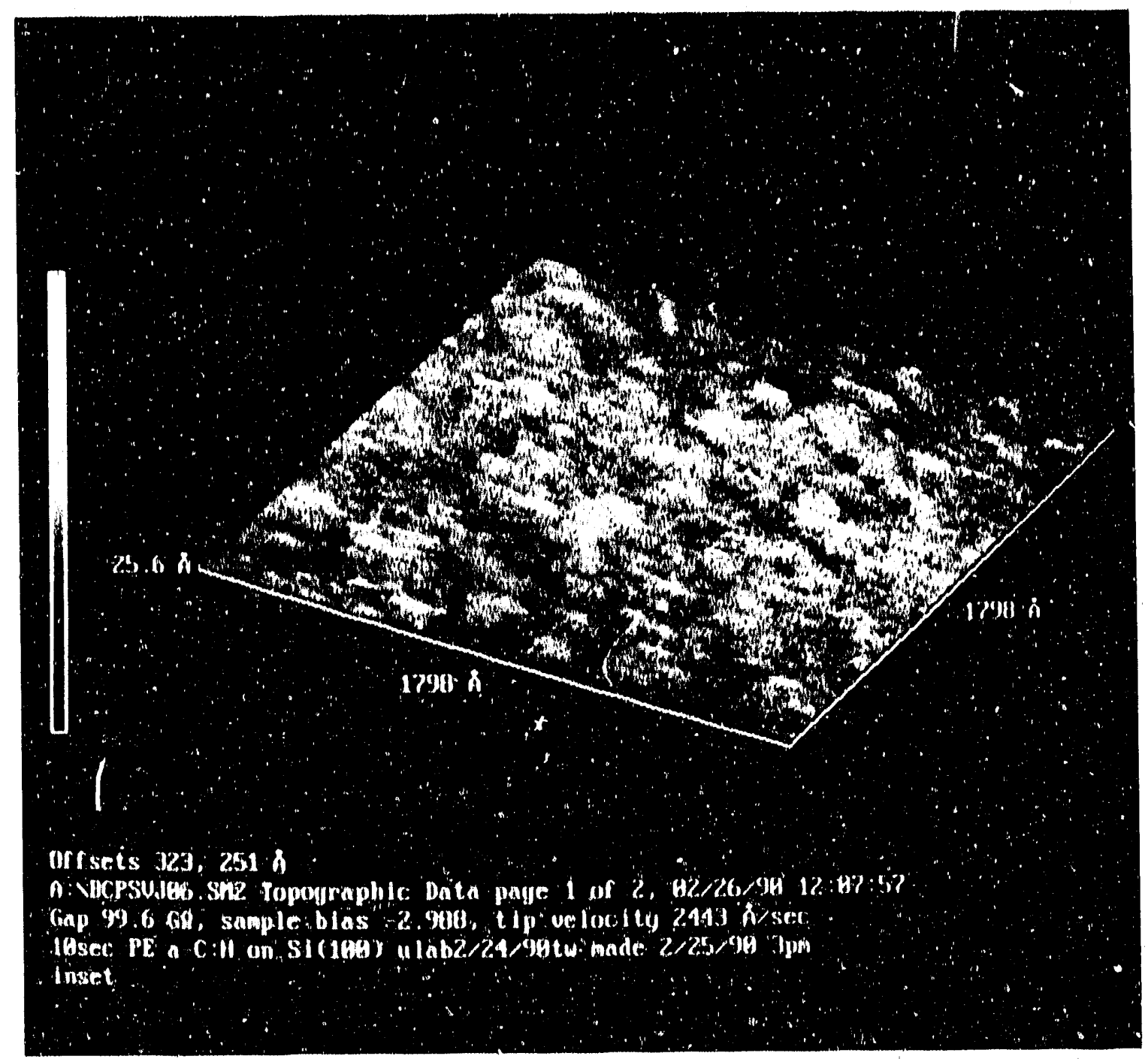

XBB 905-3955

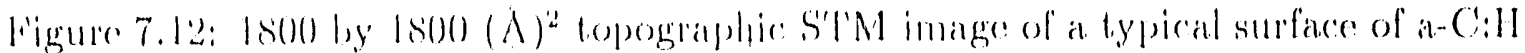

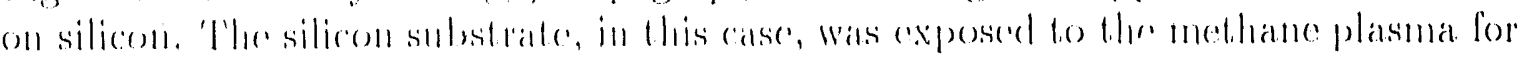

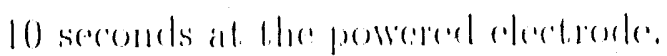


to produce nseful images. Since the material present at the grounded olectrode is most likely an insulating material poorly bonded to a somiconductor, this result is not surprising.

\subsection{Discussion}

\subsection{1 a-C:H on Eraphite}

From the results given in previous chapters, it is known that the plasma growth conditions are very different for films grown on substrates placed at the negatively self biased electrode, as compared with those grown at the grounded electrode. The most significant difference is the higher kinetic energy of ions striking the growing surface at the self biased electrode (approx. $350 \mathrm{eV}$ ), as compared to the kinetic energy of ions striking the film surface at the grounded electrode (approx. $15 \mathrm{eV}$ ). The ion flux is probably also significantly higher at the self biased electrode. In contrast, the flux of low energy neutral radical species (predominantly $\mathrm{CH}_{3}$ ), is about the same at both electrodes. Evidence suggests that the growth mechanism for these films is one where the radicals react and bond to the growing film surface to provide the bulk mass of these films by polymerization. Without high energy ion bombardment, as is the case at the grounded electrode, the films grown are soft and polymerlike. In contrast, films grown on silicon at the self biased electrode, where high energy ion bombardment is present, exhibit much different properties, such as a high hardness, and lower hydrogen content. Films formed at the self biased electrode also exhibit better adhesion onto silicon than do films formed at the grounded electrode. The high encrgy jons impinging on the substrate at the self biased electrode, contribute to the formation of a silicon carbide interface, not present in the case of soft films grown on silicon at the grounded electrode (see chapler 6 ).

In Figs. 7.6 and 7.7, a clear difference is observable between the intial stages of growth at the self biased electrode, as compared to the grounded electrode, for a-C:H films on graphite substrates. There is distinct cluster formation evident at the self biased electrode, and no such clustering is observed at the grounded electrode. In contrast, a randomly rough surface is observed at the grounded electrode with a signal to noise level noticeably reduced as compared to the self biased electrode case. 
A proposed explanation for these observations is as follows. The ideal graphite surface has no dangling bonds and is quite unreactive[8]. The sticking coefficient of $\mathrm{CH}_{n}$ radicals to an ideal graphite surface is probably extremely small. Estimates of methyl radical sticking coefficients with the reactive film surface are near $0.03[1,9]$, and even lower $(<0.001)$ as predicted for the sticking probability with the chamber walls[10]. An ideal graphite surface would likely give sticking probabilities even much smaller than these. As a result, a rapid and strong homogeneous chemical attachrnent of radicals to the graphite surface is unlikely. Additional support for this statement is the markedly small initial deposition rate of a-C:H on graphite. The average bulk deposition rate of these films on silicon is about $2 \AA / \sec [1]$. The initial deposition rate on graphite substrates is much smaller, as complete coverage does not occur over the stable graphite surface until a deposition time of more than about 20 seconds. At the average bulk deposition rate, complete coverage would have occurred much earlier.

It is proposed that a disruption of the graphite surface is necessary before plasma radicals can chemically bond to the surface. Such a disruption is provided by the high energy ions impinging on the surface at the self biased electrode. The observed clusters are formed as a result of the more rapid and irreversible bonding of reactive plasma species at the site of the ion induced defects. A cluster may reach a critical size when its surface has become depleted in active sites, or dangling bonds, and hence it would remain relatively inactive until a new disruption is formed by means of another ion bombardment event. This would explain why cluster size is relatively constant, as the density increases. At the grounded electrode, in contrast, there are comparatively few active sites where radicals can bind to the substrate. Absent completely are the disruptions created at the self biased electrode by high energy ions. Some deposition is still observed, however, as seen in Fig. 7.5. The material deposited here is likely more polymerlike, and only weakly bonded to the surface. The fact that the tunneling gap is more unstable at this surface, as compared with that at the self biased electrode, is not, incorsistent with the presence of weakly bonded species at the surface. The lack of these weakly bonded species between the clusters observed at the self biased electrode can be justified by assuming that such species are more likely to 
be desorbed from the more energetic areas surrounding the ion bombardment sites.

\subsection{2 a-C:H on silicon}

Cluster formation during the growth of a-C:H on silicon substrates at the self biased electrode was not observed, as shown in Fig. 7.12. From previous angle resolved $\mathrm{X}$-ray photoelectron spectroscopy studies, it has been confirmed that a silicon carbide interface is formed completely in less than 10 seconds of deposition timie. It is expected that, even at the shortest deposition time of 5 seconds, most of the silicon substrate has been covered up by a-C:H. Since deposition time is not controllable accurately for deposition times less than 5 seconds, expected coverages of less than about one half are not observable. If the initial deposition rate were equal to the average bulk deposition rate of about $2 \AA / \mathrm{sec}$, the point of complete coverage would certainly have becn passed in less than 5 seconds. The initial deposition rate in the case of the graphite substrates, is clearly much less than the bulk deposition rate of these films, as complete substrate coverage does not occur until after about 20 seconds. No information on the initial deposition rate of $\mathrm{a}-\mathrm{C}: \mathrm{H}$ films on silicon substrates is extractable, as the substrate surface and the film surface are indistinguishable by our current STM capabilities. An initial cluster formation, rapidly smoothed out before the shortest 5 second deposition time, can not be ruled out, however this seems unlikely.

A proposed explanation for the apparent lack of clustering on silicon is as follows. The silicon substrate surface, before deposition, is hydrogen terminated[11, 12, 13], and presumably is much more reactive towards plasma radicals than is the very stable graphite surface. The low initial deposition rate on graphite was justified by the proposed small sticking coefficient of radicals onto the graphite surface. This condition presumably does not exist on the silicon surface, and the initial growth rate could be much greater. Once an a- $\mathrm{C}: \mathrm{H}$ layer has already been formed, the final effect of high energy ion bombardment may not be apparent lopologically to the STM. The effect of the high energy ion bombardment on the hardness and other bulk properties of a-C:H films on silicon is quite clear[1], however its affect on the topology of the $\mathrm{a}-\mathrm{C}: \mathrm{H}$ film surface is not noticeable here. 
The proposed explanation is consistent with what was observed by STM and with other results in this work. It is clear that the initial stages of growth proceed by means of a complex mechanism involving both plasma ions and radicals. Investigating the initial stages of a-C:H growth on silicon at even shorter deposition times would be most interesting. A method of exposing the substrate to very short plasma interactions of less than 5 seconds, while maintaining the identical plasma conditions, would be necessary, a challenging experimental task. 


\section{Bibliography}

[1] B. Marchon, M. Salmeron, and W. Siekhaus, Phys. Rev. B 39, 17.(1989).

[2] C.W. Nelson, Data General Inc., internal report, 1973 (unpublished).

[3] K.M. Mertz, Jr., R. Hoffmann, and A.T. Balaban, J. Am. Chem. Soc. 109, 6742 (1987).

[4] H. Ibach and D.L. Mills, Electron Energy Loss Spectroscopy, (Academic Press, New York, 1985).

[5] G. Ertl and J. Kuppers, Low Energy Electrons and Surface Chemistry, (VCH, Germany, 1985).

[6] J.T. Yates and T.E. Madey (editors), Vibrational Spectroscopy of Molecules on Surfaces, (Plenum Press, New York, 1987).

[7] B.J. Waclawski, D.T. Pierce, N. Swanson, and R.J. Cotton, J. Vac. Sci. Technol. 21(2), 368 (1982).

[8] J. Carrazza, J.J. Chludzinski, Jr., H. Heinemann, G.A. Somorjai, and T.K. Baber, J. Catal. 110, 74 (1988).

[9] L.E. Kline, W.D. Partlow, and W.E. Bies, J. Appl. Phys. 65, 70(1989).

[10] H. Toyoda, H. Kojima, and H. Sugai, Appl. Phys. Lett. 54, 1507 (1989).

[11] G.S. Higashi, Y.J. Chabal, G.W. Trucks, and Krishnan Raghavachari, Appl. Phys. Lett. 56, 656 (1990).

[12] N. Hirashita, M. Kinoshita, I. Aikawa, and T. Ajioka, Appl. Phys. Lett. 56(5), $451(1990)$. 
[13] T. Takahagi, I. Nagai, A. Ishitani, H. Kuroda, and Y. Nagasawa, J. Appl. Phys. 64(7), 3516(1988).

[14] R.M. Feenstra, J.A. Stroscio, and A.P. Fein, Surface Science, 181, 295 (1987), and also R.J. Hamers, R.M. Tromp, and J.E. Demuth, Phys. Rev. Lett. 59, 2071 (1986). 


\section{Chapter 8}

\section{Summary}

The objective of this work was to develop a better understanding of the growth process of a-C:H films produced by PACVD from methane. The films are very useful as wear resistant coatings because of their desirable properties such as high hardness. In order to understand the origin of the properties of a- $\mathrm{C}: \mathrm{H}$ films, the growth process must be better understood. Once the precursor species and their roles in the growth mechanisms are elucidated, better control over the process and the film properties will be possible. Such a control would be exceedingly valuable so that limitations in the applicability of a-C:H films could be overcome, and films could be synthesized with the properties most desirable for a given application.

Initially, deposition conditions were defined to produce films with the desirable properties of high hardness and good adhesion with their silicon substrates. It was observed that films formed on the powered electrode of the parallel plate electrode system exhibited significantly superior mechanical properties, as compared with films formed at the grounded electrode. The Knoop hardness of films formed at the powered electrode was measured at $33 \mathrm{GPa}$, which is much greater than that of the films formed at the grounded electrode, measured at $2 \mathrm{GPa}$. A large amount of compressive stress was present in the powered electrode films (1.5 - 3.0 GPa), while no stress was measurable in the case of the grounded electrode films. The adhesion of the powered electrode films with the silicon substrates was also much greater than that of the grounded electrode films.

The soft grounded electrode films were found to contain much more hydrogen than the PE films, as noted by the intensity of the FTIR signal originating from C-H 
bond vibrations. A large concentration of terminal $-\mathrm{CH}_{3}$ groups was measured in the grounded electrode films, and little in the powered electrode films. This is indicative of less crosslinking in the grounded versus the powered electrode films. The optical gap of the films was also measured. That of the powered electrode films was much smaller $(1.2 \mathrm{eV})$ than that of the grounded electrode films $(3.1 \mathrm{eV})$. The decreased gap in the case of the powered electrode films is thought to result form the presence of conjugated clusters of graphitelike or $\mathrm{sp}^{2}$ carbon.

The potential profile between the electrodes of the rf plasma system displays a large potential drop in the sheath at the powered electrode. Thus the energy of ions bombarding the surfaces of the films is much greater at the powered electrode than at the grounded electrode. This fact is a valuable piece of information towards understanding the growth mechanism and the origin of the large differences in the properties of the films formed at opposite electrodes.

In order to develop a more concise understanding of the growth process and the effect of plasma conditions on film properties, the chemical species impinging on the film surface were studied by mass spectroscopy. Both the identity and the flux of the ionic and neutral species were measured and compared to each other and the deposition rate.

The dominant radical species was measured to be the methyl radical. Its flux onto the film surface was found to be approximately the same at both the powered and grounded electrode. Since the sticking coefficient of the methyl radical with the stainless steel walls of the chamber was found to be quite small, the flux of methyl radicals onto the surface could be determined. It was calculated to be approximately $7 \times 10^{16} \mathrm{~cm}^{-2} \mathrm{~s}^{-1}$. The deposition rate of the a-C:H films was estimated to be 1.9 $\times 10^{15} \mathrm{~cm}^{-2} \mathrm{~s}^{-1}$, so that the methyl radical flux was sufficient to account for the deposition rate. If the methyl radicals were the exclusive precursor species, their sticking probability with the growing film surface would be 0.02 to 0.03 .

The ionic species incident on the electrodes were found to be significantly different in identity and in relative flux, between the powered and grounded electrodes. Fewer secondary species (created through ion - molecule reactions) are present at the powered electrode, as ions are accelerated out of the plasma very quickly here due to the 
large potential drop in the sheath. An upper limit on the total ion flux was measured using a Faraday cup. It was measured as $1.7 \times 10^{15} \mathrm{~cm}^{-2} \mathrm{~s}^{-1}$ at the powered electrode and $0.4 \times 10^{15} \mathrm{~cm}^{-2} \mathrm{~s}^{-1}$ at the grounded electrode. At both electrodes, the ion flux measured was insufficient to account for the total deposition rate.

The most significant results of the plasma mass spectrometric studies are as follows: The methyl radical flux was at least 40 times greater than the total ion flux, and was large enough to account for the deposition rate. If methyl radicals were the exclusive precursor, their sticking probability would be 0.02 to 0.03 . The total ion flux was insufficient for ions to be the predominant precursor species in a-C:H deposition. Methyl radicals could not have been responsible for the large differences in the properties of films formed at the powered and grounded electrodes, since the methyl radical flux did not differ appreciably between electrodes. Ion flux and ion energy were much greater at the powered electrode, as compared to the grounded electrode. Although ions could not have been the main film precursor species, they could have been responsible for the difference in film properties between the two electrodes.

In order to determine the relative roles of ions and radicals in the growth mechanism of a-C:H films, experiments were designed to control the flux of these species independently. The ion energy flux was controlled independently of the radical flux using a novel pulsed biasing technique. Film properties such as hardness, stress, optical gap, and $-\mathrm{CH}_{3}$ and $-\mathrm{CH}_{2}$ - concentration, varied smoothly with varying ion energy flux but not with the individual energy of the ions. Thus the flux of energy transferred to the surface of the growing films was found to be the most significant variable controlling the final properties of the films. Radical scavenging experiments demonstrated that removal of radicals from the plasma resulted in a rapid drop in the deposition rate. Thus the relative roles of ions and radicals in the growth mechanism of a-C:H films were determined. Radicals acted as the main deposition species responsible for the bulk mass growth of the films, while ion bombardment acted to control film properties.

Films formed at the grounded electrode, where ion bombardment is relatively weak, were polymerlike and soft. They exhibited a large optical gap, and a high hydrogen content. As ion bombardment was increased using the pulsed biasing tech- 
nique, the structure of the films changed. Less hydrogen was incorporated into the films and the hardness and density of the films increased. The optical gap of the films decreased, presumably due to the formation of small domains of $\mathrm{sp}^{2}$ or graphite like carbon. The properties of the films varied with the ion energy flux but not with the energy of the individual ion. A mechanism involving large but local thermal spikes, quenched rapidly by the bulk of the film, as proposed by Weissmantel is consistent with this observation. Within energetic spike regions, processes altering the structure and properties of the deposited material could occur, such as bond rearrangement, cross linking, and hydrogen elimination. Such processes must be active in the structural and physical changes observed in the films as a result of ion bombardment. Films formed by radical deposition with a minimal amount of ion bombardment were soft and polymerlike, and poorly adherent to silicon substrates. With high energy ion bombardment, very hard films were be formed. Other film properties, such as compressive stress and optical gap changed concurrently with changes in ion energy flux. The technique of pulsed biasing provides a means of control of ion energy flux, and thus provides a convenient means for controlling film properties.

The effect of high energy ion bombardment on the interface of a- $\mathrm{C}: \mathrm{H}$ films grown on silicon substrates was studied by angle resolved XPS. A silicon carbide interfacial layer was found to exist for films formed on the powered electrode. No such silicon carbide layer was found to be present in the case of films formed at the grounded electrode, where high energy ion bombardment was absent. The thickness of the interfacial layer was found to be about $7 \AA$ for films formed at an rf power of 50 $\mathrm{W}$, where the self bias voltage was $-360 \mathrm{~V}$. The thickness of the interfacial layer was found to change with self bias voltage, or ion energy. Films formed at higher self bias voltage, and thus subjected to bombardment by ions of a higher energy, were found to have a thicker silicon carbide layer present at the interface. The measured thickness of the interface varied in a way similar to the way in which average ion penetration depth varies with ion energy. The same effect was observed for films formed under pulsed biasing conditions. Thus it was determined that high energy ion bombardment was responsible for the formation of an interfacial layer of silicon carbide. Films formed at the powered electrode were found to exhibit much better adhesion to the silicon 
substrate, as compared to films formed at the grounded electrode. The silicon carbide interfacial layer was likely responsible for this strong adhesion. At the grounded electrode, where adhesion was poor, no silicon carbide layer was present.

SIMS depth profiles were carried out in order to reproduce work done by others us. ing ion depth profiling. It was found that the depth profling technique of SIMS lacked the necessary depth resolution to distinguish between the interfaces of films formed on the grounded and powered electrodes. An apparent interface of approximately 100 $\AA$ thickness was measured for both grounded electrode and powered electrode films. These results agreed with those previously published, which claimed that a silicon carbide interfacial layer existed in the case of both PE and GE films. The XPS results demonstrated how this was not the case, as no silicon carbide is formed at the grounded electrode, and in fact, the silicon carbide layer for films formed at the powered electrode was indeed much thinner than thicknesses which could be measured by ion depth profiling techniques.

Scanning tunneling microscopy was used to study the initial stages of growth of a-C:H films on graphite and silicon substrates. On graphite, at the self biased electrode, where high energy ion bombardment occurs, the films were observed to nucleate in clusters, possibly nucleating at ion induced darnage sites. Cluster size remained constant, at approximately $50 \AA$ in diameter, and cluster density increased with deposition time. On graphite at the grounded electrode, where high energy ion bombardment is absent, no clustering was observed. a- $\mathrm{C}: \mathrm{H}$ films grown on silicon substrates at the self biased electrode, were observed to have a very smooth topology ( $<3 \AA$ roughness) and no cluster formation was observed. This more homogeneous growth is likely a consequence of the greater reactivity of the silicon surface with plasma species, as compared with that of the graphite surface. Novel $2 \times 2$ structures were imaged at the surface of graphite samples which had been exposed to the methane plasma.

In summary, a great deal has been learned about the growth mechanisms of a. $\mathrm{C}: \mathrm{H}$ films synthesized by PACVD from methane. Novel techniques have been introduced in order to study the plasma and control plasma species independently. The complimentary roles of plasma radicals and ions as bulk precursor species and structural 
modiffers, respectively, have been clearly elucidated. The effect of ion bombardment in creating an interfacial carbide layer which results in strong adhesion has also been demonstrated. The nucleation structures during the initial stages of $a-C: H$ films have been imaged using scanning tunneling microscopy. A much deeper understanding of the chemical processes occurring in the formation of a-C:H films from methane by PACVD has been achieved. 

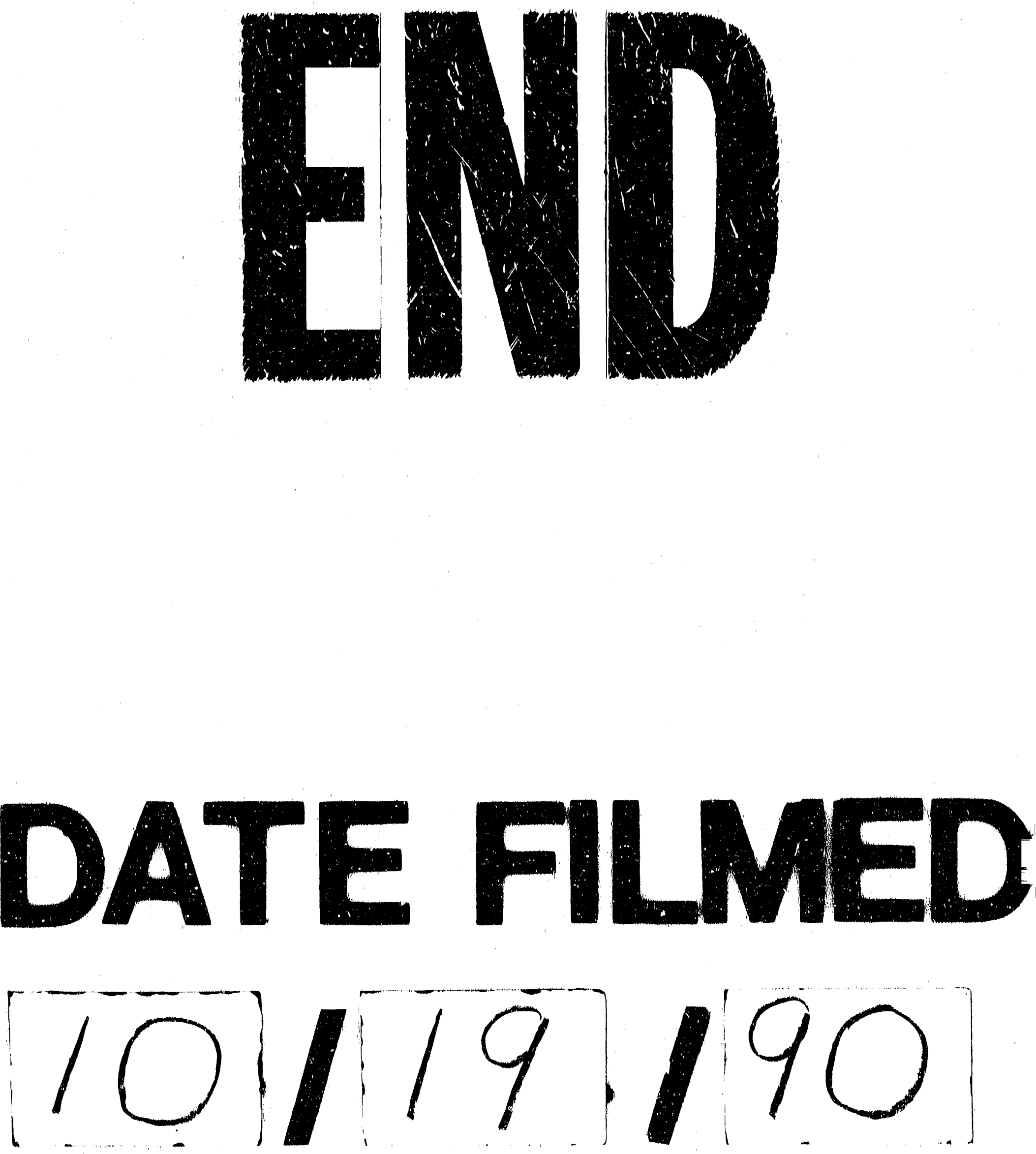
